\title{
SUB-RIEMANNIAN SPHERE IN MARTINET FLAT CASE
}

\author{
A. AGRACHEV, B. BONNARD, M. CHYBA, AND I. KUPKA
}

\begin{abstract}
This article deals with the local sub-Riemannian geometry on $\mathbb{R}^{3},(D, g)$ where $D$ is the distribution ker $\omega, \omega$ being the Martinet one-form: $d z-\frac{1}{2} y^{2} d x$ and $g$ is a Riemannian metric on $D$. We prove that we can take $g$ as a sum of squares $a d x^{2}+c d y^{2}$. Then we analyze the flat case where $a=c=1$. We parametrize the set of geodesics using elliptic integrals. This allows to compute the exponential mapping, the wave front, the conjugate and cut loci, and the sub-Riemannian sphere. A direct consequence of our computations is to show that the sphere is not sub-analytic. Some of these computations are generalized to a one parameter deformation of the flat case.
\end{abstract}

\section{INTRODUCTION}

In this article we consider the sub-Riemannian geometry $(M, D, g)$ where $M$ is the real analytic manifold $\mathbb{R}^{3}, D$ is the distribution ker $\omega, \omega$ being Martinet one-form $d z-\frac{1}{2} y^{2} d x$, where $q=(x, y, z)$ are the coordinates of $\mathbb{R}^{3}$ and $g$ is an analytic Riemannian metric on $D$. Since on $D, d z=\frac{1}{2} y^{2} d x, g$ can be written $a(q) d x^{2}+2 b(q) d x d y+c(q) d y^{2}$. When $a, b, c$ are not depending on $z$ the problem is said isoperimetric.

An admissible curve is an absolutly continuous curve $\gamma:[0, T] \longmapsto \mathbb{R}^{3}$ such that $\dot{\gamma}(t)=\frac{d \gamma(t)}{d t} \in D(\gamma(t)) \backslash\{0\}$ for almost every $t$. Let $($,$) be the$ scalar product defined by $g$. The length of an admissible curve is:

$$
L(\gamma)=\int_{0}^{T}(\dot{\gamma}(t), \dot{\gamma}(t))^{\frac{1}{2}} d t
$$

and the energy of $\gamma$ is:

$$
E(\gamma)=\int_{0}^{T}(\dot{\gamma}(t), \dot{\gamma}(t)) d t
$$

Let $q_{0}, q_{1} \in \mathbb{R}^{3}$, a minimizing curve joining $q_{0}$ to $q_{1}$ is an admissible curve $\gamma:[0, T] \longmapsto \mathbb{R}^{3}$ joining $q_{0}$ to $q_{1}$ with minimal length and the length of $\gamma$ defines the sub-Riemannian distance $d_{S R}$ between $q_{0}$ and $q_{1}$.

Andrei Agrachev, Steklov Mathematical Institute, 42 Vavilova Street, GSP-1, Moscow 117966 Russia. E-mail: agrachev@mi.ras.ru.

Bernard Bonnard, Université de Bourgogne, Laboratoire de Topologie, UMR 5584 du CNRS, BP 400, 21004 Dijon Cedex France. E-mail: bbonnard@u-bourgogne.fr.

Monique Chyba, Section de Mathématiques, Université de Genève, 2-4 rue du Lièvre, Case 240, 1211 Genève 24, Suisse. E-mail: chybađsunny -unige.ch.

Ivan Kupka, Université de Paris VI, Département de math., 4, place Jussieu, 75232 Paris Cedex 05, France. E-mail: kupka@mathp6.jussieu.fr.

Received by the journal November 22, 1996. Revised November 10, 1997. Accepted for publication November 28, 1997.

(C) Société de Mathématiques Appliquées et Industrielles. Typeset by IATEX. 
In the Martinet flat case, a standard existence theorem due to Filippov [26] and the maximum principle [32] allows to show the existence of such minimizer in the analytic category. Hence in the sequel we shall restrict our study to analytic admissible curves.

Let $F_{1}, F_{2}$ be two analytic vector fields such that $D=\operatorname{Span}\left\{F_{1}, F_{2}\right\}$. Hence an admissible (analytic) curve is solution of the control system:

$$
\frac{d \gamma(t)}{d t}=\sum_{i=1}^{2} u_{i}(t) F_{i}(\gamma(t))
$$

where $u:[0, T] \longmapsto\left(u_{1}(t), u_{2}(t)\right)$ is the unique analytic control associated to $\gamma$. The two following remarks can save a lot of computations in subRiemannian geometry. First, the length of a curve is not depending on its parametrization. Hence, we can assume the curves parametrized by arclength

$$
(\dot{\gamma}(t), \dot{\gamma}(t))=1
$$

and in this case, the length minimizing problem is equivalent to the time optimal control problem. Secondly, if all the curves are defined on the same interval, the length and energy minimizing problems are equivalent.

To carry out the computations of minimizers, it is convenient to use maximum principle. According to this principle, minimizers can be selected among a restricted set of curves. Let us introduce the Hamilton function:

$$
H_{\lambda}(q, p, u)=\langle p, F(q) u\rangle-\lambda(F(q) u, F(q) u)
$$

where $p=\left(p_{x}, p_{y}, p_{z}\right) \in \mathbb{R}^{3}, \lambda$ is a constant equals to 0 or $\frac{1}{2},(p, \lambda)$ is non zero, $\langle$,$\rangle is the standard scalar product and F(q) u$ denotes $\sum_{i=1}^{2} u_{i} F_{i}(q)$. A bi-extremal is an absolutly continuous curve $(\gamma, p)$, defined on $[0, T]$ where $(\gamma, p, u)$ is solution for almost every $t$ of the following equations

$$
\begin{aligned}
\frac{d \gamma}{d t}=\frac{\partial H_{\lambda}}{\partial p}(\gamma, p, u), \frac{d p}{d t} & =-\frac{\partial H_{\lambda}}{\partial \gamma}(\gamma, p, u) \\
\frac{\partial H_{\lambda}}{\partial u}(\gamma, p, u) & =0 .
\end{aligned}
$$

Its projection $\gamma$ on $\mathbb{R}^{3}$ is called a geodesic. From the maximum principle, a minimizer is a geodesic.

When we study the bi-extremal curves, we must distinguish between two cases. If $\lambda=0$, the bi-extremal is called abnormal and if $\lambda \neq 0$, it is called normal and the associated geodesics are respectively called abnormal and normal. A geodesic is called strictly abnormal (respectively strictly normal) if it is the projection of an abnormal (respectively normal) bi-extremal but not of a normal (respectively abnormal) one.

In the normal case, equation (1.2) can be solved as follows. Since it is linear with respect to $u$, the control solution can be computed as an analytic maping $\hat{u}:(\gamma, p) \longmapsto \mathbb{R}^{2}$. We plug $\hat{u}$ in $H_{\lambda}$, and we set $H_{n}(\gamma, p)=$ $H_{\lambda}(\gamma, p, \hat{u})$, where $\lambda=\frac{1}{2}$. Now using (1.1) and (1.2), we observe that the biextremal $(\gamma, p)$ is solution of the analytic differential Hamiltonian equation

$$
\frac{d \gamma}{d t}=\frac{\partial H_{n}}{\partial p}(\gamma, p), \frac{d p}{d t}=-\frac{\partial H_{n}}{\partial \gamma}(\gamma, p)
$$


This computation is straightforward if $F_{1}, F_{2}$ are taken orthonormal. If we set $P_{i}=\left\langle p, F_{i}(q)\right\rangle$ for $i=1,2$, the Hamilton function $H_{n}$ takes the form $\frac{1}{2}\left(P_{1}^{2}+P_{2}^{2}\right)$.

Similarly, the computations of abnormal bi-extremals is straightforward. When $\lambda=0$, the constraint (1.2) is

$$
\left\langle p, F_{i}(\gamma)\right\rangle=0, i=1,2
$$

and if we differentiate twice equation (1.4), we get that abnormal geodesics are contained in the set $\operatorname{det}\left(F_{1}, F_{2},\left[F_{1}, F_{2}\right]\right)=0$ which is the plane $y=0$ corresponding to the points where $\omega$ is not a contact form and are the lines $z=z_{0}$. The set $y=0$, which has an important geometric meaning is called the Martinet surface.

Consider now arc-length parametrized curves. We fix $q_{0} \in \mathbb{R}^{3}$ and let $\gamma\left(t, q_{0}, p_{0}\right), p\left(t, q_{0}, p_{0}\right)$ be the solution of $(1.3)$ starting at $t=0$ from $\left(q_{0}, p_{0}\right)$. It is contained in the level set $H_{n}=\frac{1}{2}$. The exponential mapping is the map:

$$
\exp _{q_{0}}:\left(p_{0}, t\right) \longmapsto \gamma\left(t, p_{0}, q_{0}\right) .
$$

The point $q_{1}$ is said to be conjugate to $q_{0}$ along $\gamma$ if there exists $\left(p_{0}, t_{1}\right)$ such that $\gamma(t)=\exp _{q_{0}}\left(p_{0}, t\right), q_{1}=\exp _{q_{0}}\left(p_{0}, t_{1}\right), t_{1}>0$ and the exponential mapping is not an immersion at $\left(p_{0}, t_{1}\right)$. The conjugate locus $C\left(q_{0}\right)$ is the set of first conjugate points along the curves $\gamma$, when we consider all the geodesics starting from $q_{0}$. Observe that the exponential mapping is defined on $C \times \mathbb{R}$, where $C$ is the cylinder parametrized by $P_{1}^{2}+P_{2}^{2}=1$ at $q_{0}$, if $F_{i}$ are taken orthonormal. The non compact nature of $C$ is the main problem when we study the exponential mapping. Let $\gamma$ be a geodesic corresponding to a normal or an abnormal bi-extremal and starting from $q_{0}$. The first point $q_{1} \neq q_{0}$ where $\gamma$ ceases to be minimizing is called the cut point and the set of such points when $\gamma$ varies form the cut-locus $L\left(q_{0}\right)$. The subRiemannian sphere with radius $r>0$ is the set $S\left(q_{0}, r\right)$ of points which are at sub-Riemannian distance $r$ from $q_{0}$. The wave front, of length $r$, is the set $W\left(q_{0}, r\right)$ of end-points of geodesics with length $r$ starting from $q_{0}$.

One of the main problem in sub-Riemannian geometry is the study of the exponential mapping in order to give estimates and a geometric description of the conjugate and cut loci, of the sphere and the wave front. Related works to this problem are the following. In a pionnering contribution [11], Brockett has analyzed the Heisenberg case, where $M$ is the 3-dimensional Heisenberg group, $D$ is a left invariant contact distribution and $g$ is left invariant. Very recently, the sub-Riemannian sphere with small radius was computed, when $D$ is ker $\omega, \omega$ being a contact form in $\mathbb{R}^{3}$ and $g$ is a generic metric [2], [17]. It appears that the general case is a perturbation of the Heisenberg case. The object of this article is to pursue the analysis by considering the Martinet situation where $D=\operatorname{ker} \omega, \omega$ being $d z-\frac{1}{2} y^{2} d x$.

The main results of this article are threefolds.

First we clarify the geometry of the problem by computing a normal form for the pair $(D, g)$, the group action $G$ being induced by the following two set of transformations

(i) $q \longmapsto Q=\varphi(q)$ where $\varphi$ is a germ of analytic diffeomorphism on $\mathbb{R}^{3}$ preserving 0 ; 
(ii) feedback transformation of the form $u \longmapsto v=\theta(q) u$, where $q \longmapsto$ $\theta(q) \in G L(2, \mathbb{R})$, is a germ at 0 of an analytic mapping, and is preserving the metric $g$. If $D=\operatorname{Span}\left\{F_{1}, F_{2}\right\}$, where $F_{1}, F_{2}$ are orthonormal vector fields, $\theta(q) \in O(2)$ the set of orthogonal matrices.

We can formulate a first main result.

Theorem 1.1. Let $(D, g)$ be such that $D=\operatorname{ker} \omega, \omega=d z-\frac{1}{2} y^{2} d x$ and $g$ is an analytic germ at 0 of a Riemannian metric on $D: a(q) d x^{2}+$ $2 b(q) d x d y+c(q) d y^{2}$. Then under the action of $G$, we may assume $b \equiv 0$ and $a(0)=c(0)=1$.

Technically the proof of this result is relevant for two reasons. First, we need to parametrize the set of germs of diffeomorphisms preserving the distribution ker $\omega$ and we improve a similar result obtained in [33], where only those tangent to the identity are computed. This parametrization is important to any geometric problem dealing with Martinet type distributions. Secondly, we show that the problem of computing our normal form is related to a Cauchy problem for a singular partial differential equation of the Briot-Bouquet type. This type of problem is studied in [18] and it allows to use a Cauchy-Kowaleska type theorem to get a convergent normal form in our situation.

In this normal form, the distribution is entirely normalized. Let us introduce the two vector fields: $F_{1}=\frac{\partial}{\partial x}+\frac{y^{2}}{2} \frac{\partial}{\partial z}$ and $F_{2}=\frac{\partial}{\partial y}$ such that $D=\operatorname{Span}\left\{F_{1}, F_{2}\right\}$. Hence the length of $F_{1}, F_{2}$ is given by

$$
\left(F_{1}, F_{1}\right)=a,\left(F_{2}, F_{2}\right)=c
$$

and we get two orthonormal vector fields by setting

$$
\widetilde{F}_{1}=F_{1} / \sqrt{a}, \quad \widetilde{F}_{2}=F_{2} / \sqrt{c}
$$

and the two vector fields $\left(\widetilde{F}_{1}, \widetilde{F}_{2}\right)$ are a representation of $(D, g)$. Using the following gradation for the variables: the weight of $x, y$ is one and the weight of $z$ if three, the associated weights for vector fields being -1 for $\frac{\partial}{\partial x}$, $\frac{\partial}{\partial y}$ and -3 for $\frac{\partial}{\partial z}$, we get a gradated normal form using $\left(\widetilde{F}_{1}, \widetilde{F}_{2}\right)$. With this gradation, the flat case $g=d x^{2}+d y^{2}$ corresponds precisely to collect only the terms of weight -1 in the normal form. Hence in terms of normal form, the flat case in the Martinet case is the equivalent of the Heisenberg case when we analyse the contact situation as in [2], [17].

Therefore, the Martinet flat case has to be carefully studied. The second part of our work is to get a complete descrition of the conjugate and cut loci and of the sphere in this situation. We prove

ThEOREM 1.2. The intersection of the conjugate-locus $C(0)$ and the cutlocus $L(0)$ is empty. The cut locus $L(0)$ is the Martinet surface $y=0$ minus the abnormal geodesic $z=0$. The intersection of the sphere $S(0, r), r>0$ with the Martinet surface $y=0$ is a closed cuve $k \longmapsto c(k)$ around 0 which is the union of $L(0)$ intersected with the sphere and two points $x= \pm r, z=0$ representing the trace of the abnormal geodesic on the sphere. The graph of $c$ is not sub-analytic. Hence the sphere and the sub-Riemannian distance are not sub-analytic.

Esaim: Cocv, December 1997, Vol. 2, PP. 377-448 
This result illustrates the main difference between the contact and the Martinet case. In the Martinet case there exist abnormal geodesics. One object of this article is to prove that abnormal geodesics will cause a logarithmic singularity for the exponential mapping. The main technical tool to handle this problem is to use models where the set of geodesics can be computed by quadratures. In the Martinet flat case we need elliptic integrals. It is a precise measure of the transcendence of the problem. Using the catalogue of properties and estimates concerning elliptic integrals, we shall be able to estimate the conjugate-locus and to compute the cut-locus. Indeed our study will show that the cut-locus has a singularity similar to the singularity of Poincaré-Dulac return mapping for planar algebraic differential equations when computed for a section to a separatrix connecting two saddles $[22]$.

The Martinet flat case is not a stable model to understand in general the role of abnormal geodesics in sub-Riemannian geometry when $D$ is a Martinet type distribution. Indeed in the flat case an abnormal geodesic is not strictly abnormal. Hence a third contribution of our work is to build a one-parameter deformation of the flat case $\left(D_{\varepsilon}, g_{\varepsilon}\right)$ where for $\varepsilon \neq 0$, each abnormal geodesic is strictly abnormal. The deformation is constructed in order to get the set of geodesics integrable by quadratures. If we require to have a parametrization of geodesics with the lowest transcendence (this shall be precised later), the model chosen is

$\left(D_{\varepsilon}, g_{\varepsilon}\right)$ where $D_{\varepsilon}=\operatorname{Span}\left\{F_{1}, F_{2}\right\}, F_{1}=(1+\varepsilon y) \frac{\partial}{\partial x}+\frac{y^{2}}{2} \frac{\partial}{\partial z}$ and $F_{2}=\frac{\partial}{\partial y}$,

and the metric $g_{\varepsilon}$ is defined by taking $F_{1}$ and $F_{2}$ as orthonormal vector fields. In this model the Martinet surface is the set $y=0$, when $|y|<\frac{2}{1 \varepsilon}$ and the abnormal geodesics are the lines $y=0, z=z_{0}$. If $\gamma$ is the abnormal geodesic starting from 0 and parametrized by arc-length: $t \longmapsto( \pm t, 0,0)$ we prove the following result.

Theorem 1.3. Let $M \geq 0$. Then the abnormal geodesic $\gamma: t \mapsto( \pm t, 0,0)$ is $C^{0}$-isolated in the set of geodesics of length less than $M$.

A similar result has been obtained in the unpublished preprint [23], but we use a simpler model which shortens the proof. Also we evaluate the exponential mapping in the neighborhood of the abnormal geodesic. In particular we show the persistence for $\varepsilon \neq 0$ of the logarithmic term which causes the fact that the sphere is not sub-analytic in the Martinet flat case. Besides it will allow to evaluate the trace of the sub-Riemannian sphere with the Martinet plane $y=0$, near the abnormal geodesic.

These three theorems are a first step in order to understand the general case. Our approach is a mechanical approach to the problem founded on an analysis of the whole set of geodesics which are solutions of a differential equation integrable by quadratures. This approach has the advantage to select geodesics which play a central role in our analysis. Of course, it has to be completed by purely analytical methods to analyze the general situation. It will be the purpose of a series of forthcoming articles.

The organisation of this article is the following. In section 2, we compute a normal form for the sub-Riemannian geometry $(D, g)$, where $D$ is a Martinet 
type distribution. In section 3 we present two geometric properties which are elementary but crucial in the effective computation of the conjugate locus. In section 4, we analyse the Martinet flat case: we parametrize the set of geodesics and we evaluate the conjugate locus. Then we describe the cut-locus and the sphere. The section 5 is devoted to the analysis of a one-parameter deformation of the flat case. Endly an appendix contains commented numerical simulations concerning the conjugate locus and the sub-Riemannian sphere using algorithms related to our work.

\section{Sub-Riemannian geometry with a Martinet type DISTRIBUTION}

The analysis of a geometry contains three steps: compute a complete set of invariants, find a normal form and analyze the structure of the orbits set. The section is devoted to the first two steps in a sub-Riemannian geometry with a Martinet type distribution.

\subsection{Geodesics}

Definition 2.1. Consider a control system defined on an open set $U \subset \mathbb{R}^{n}$ :

$$
\frac{d x(t)}{d t}=\sum_{i=1}^{m} u_{i}(t) F_{i}(x(t))
$$

where $x=\left(x^{1}, \ldots, x^{n}\right)$ are the coordinates in $\mathbb{R}^{n},\left\{F_{1}, \ldots, F_{m}\right\}$ are $m C^{\infty}$ independant vector fields on $U$ and the set of admissible controls $\mathcal{U}$ is the set of smooth mappings $u:[0, T(u)] \rightarrow \mathbb{R}^{m}$. Let $D$ be the distribution $\operatorname{Span}\left\{F_{1}, \ldots, F_{m}\right\}$. A smooth sub-Riemannian metric $g$ is a $C^{\infty}$ positive definite quadratic form on $D$. To compute geodesics it is convenient to assume that the $F_{i}$ are taken orthonormal. The length and the energy of a smooth admissible curve are then

$$
L(\gamma)=\int_{0}^{T}\left(\sum_{i=1}^{m} u_{i}^{2}(t)\right)^{\frac{1}{2}} d t, E(\gamma)=\int_{0}^{T} \sum_{i=1}^{m} u_{i}^{2}(t) d t
$$

To compute the geodesics we shall use the following notations: $T^{*} U$ is the cotangent space and $(x, p)$ its coordinates, where $p=\left(p_{1}, \ldots, p_{n}\right)$. The Liouville form is the form on $T^{*} U: \alpha=\sum_{i=1}^{n} p_{i} d x^{i}$ and $T^{*} U$ is endowed with its canonical structure defined by the two form $\omega=d \alpha$. Let $H: T^{*} U \longmapsto \mathbb{R}$ be a smooth function, $\vec{H}$ denote the Hamiltonian vector field defined by $i_{\vec{H}}(\omega)=-d H$. If $H_{1}, H_{2}$ are smooth functions on $T^{*} U$ the Poisson-bracket is $d H_{1}\left(\vec{H}_{2}\right)=\left\{H_{1}, H_{2}\right\}$.

Having restricted $U$ if necessary, let $\left\{F_{m+1}, \ldots, F_{n}\right\}$ be $n-m$ smooth vector fields on $U$ such that for each $x \in U, \operatorname{Span}\left\{F_{i}(x) ; i=1, \ldots, n\right\}=T_{x} U$, tangent space at $x$. We introduce the $n$ functions on $T^{*} U, P_{i}=\left\langle p, F_{i}(x)\right\rangle$, where $\langle$,$\rangle is the standard inner product and we set$

$$
H_{\lambda}(x, p, u)=\sum_{i=1}^{m} u_{i} P_{i}+\lambda \sum_{i=1}^{m} u_{i}^{2}
$$

where $\lambda$ is a scalar equal to 0 or $-\frac{1}{2}$ and the vector $(p, \lambda)$ is non zero. 
Definition 2.2. A (smooth) bi-extremal is a curve $t \longmapsto(x(t), p(t))$ on $T^{*} U$ such that $(x(),. p(),. u()$.$) is solution of the following equations$

$$
\begin{gathered}
\frac{d x}{d t}=\frac{\partial H_{\lambda}}{\partial p}, \frac{d p}{d t}=-\frac{\partial H_{\lambda}}{\partial x} \\
\frac{\partial H_{\lambda}}{\partial u}=0 .
\end{gathered}
$$

It is called normal if $\lambda=-\frac{1}{2}$ and abnormal if $\lambda=0$. Its projection on the $x-$ space is called a normal (resp. abnormal) geodesic. A geodesic can be both normal and abnormal. If it is a projection of an abnormal bi-extremal but not a normal one it is called strictly abnormal. It is called strictly normal if it is not abnormal.

Proposition 2.3. Let $(x, p)$ be a normal bi-extremal. Then the corresponding control can be written $\hat{u}=\left(P_{1}, \ldots, P_{m}\right)$ and the curve $(x, p)$ is solution of the Hamiltonian vector field $\vec{H}_{n}$, where $H_{n}=\frac{1}{2} \sum_{i=1}^{m} P_{i}^{2}$. Hence $H_{n}$ is a first integral and arc-length parametrized bi-extremals are contained in the level set $H_{n}=\frac{1}{2}$.

Proof. From $\frac{\partial H_{\lambda}}{\partial u}=0, \lambda=-\frac{1}{2}$, we get $u_{i}=P_{i}$, for $i=1, \ldots, m$. Replacing $u_{i}$ by $P_{i}$ in $H_{\lambda}$, we obtain $H_{\lambda}=\frac{1}{2} \sum_{i=1}^{m} P_{i}^{2}$. Since $\frac{\partial H_{\lambda}}{\partial u}=0$, the curve $t \longmapsto(x(t), p(t))$ is solution of the Hamiltonian vector field.

Proposition 2.4. Assume $m=2$. Let $S_{i}=\left\{(x, p) \in T^{*} U ;\left\{\left\{P_{1}, P_{2}\right\}, P_{i}\right\}=\right.$ $0\}$ for $i=1,2$ and set $S=S_{1} \cap S_{2}$. Let $z: t \longmapsto(x(t), p(t))$ be an abnormal bi-extremal such that $\dot{x}(t) \neq 0$ for each $t$ and the curve $t \rightarrow z(t)$ is contained in $T^{*} U \backslash S$. Then the control $u=\left(u_{1}, u_{2}\right)$ can be computed as the unique solution on the projective space $P^{2}$ of the equation

$$
u_{1}\left\{\left\{P_{1}, P_{2}\right\}, P_{1}\right\}(z)+u_{2}\left\{\left\{P_{1}, P_{2}\right\}, P_{2}\right\}(z)=0 .
$$

If we set $H_{a}=P_{1}\left\{\left\{P_{1}, P_{2}\right\}, P_{2}\right\}-P_{2}\left\{\left\{P_{1}, P_{2}\right\}, P_{1}\right\}$, then $t \longmapsto z(t)$ is a reparametrized solution of $\vec{H}_{a}$ starting at $t=0$ from the set

$$
P_{1}=P_{2}=\left\{P_{1}, P_{2}\right\}=0 \text {. }
$$

Proof. Since $\lambda=0, H_{\lambda}$ is reduced to $\sum_{i=1}^{2} u_{i} P_{i}$ and $\frac{\partial H_{\lambda}}{\partial u}=0$ implies

$$
P_{1}(z(t))=P_{2}(z(t))=0 .
$$

Differentiating with respect to $t$ and using $u(t) \neq 0$, we get

$$
\left\{P_{1}, P_{2}\right\}(z(t))=0 \text {. }
$$

If we differentiate once more, we obtain the equation

$$
\sum_{i=1}^{2} u_{i}(t)\left\{\left\{P_{1}, P_{2}\right\}, P_{i}\right\}(z(t))=0 .
$$

Since $t \longmapsto z(t)$ lies in $T^{*} U \backslash S$, it can be solved as follows. One may assume $\left\{\left\{P_{1}, P_{2}\right\}, P_{1}\right\}(z(t)) \neq 0$, hence

$$
u_{1}=-\frac{u_{2}\left\{\left\{P_{1}, P_{2}\right\}, P_{2}\right\}}{\left\{\left\{P_{1}, P_{2}\right\}, P_{1}\right\}} .
$$

Therefore $t \longmapsto z(t)$ is a reparametrized solution corresponding to the vector field $\vec{H}_{a}$. 


\subsection{Computations in the Martinet case.}

Definition 2.5. We denote by $q=(x, y, z)$ the coordinates in $\mathbb{R}^{3}, p=\left(p_{x}\right.$, $\left.p_{y}, p_{z}\right)$ being the dual variable. The Lie bracket of two smooth vector fields $F, G$ is computed with the convention

$$
[F, G](q)=\frac{\partial F}{\partial q}(q) G(q)-\frac{\partial G}{\partial q}(q) F(q) .
$$

In particular if $P_{i}=\left\langle p, F_{i}(q)\right\rangle$, one has the identity $\left\{P_{1}, P_{2}\right\}=\left\langle p,\left[F_{1}, F_{2}\right]\right\rangle$. Let $F_{1}, F_{2}$ be the germs at 0 of two smooth vector fields and set $D=$ $\operatorname{Span}\left\{F_{1}, F_{2}\right\}$. Assume $D$ is of rank 2 at 0 . The point 0 is called a Darboux point if the vector fields $F_{1}, F_{2},\left[F_{1}, F_{2}\right]$ are independant at 0 . Then $D=$ ker $\omega$, where $\omega$ is a contact form. The point 0 is called a Martinet point if

(i) $F_{1}, F_{2},\left[F_{1}, F_{2}\right]$ are dependant at 0 ,

(ii) one of the two determinants $D_{i}=\operatorname{det}\left(F_{1}, F_{2},\left[\left[F_{1}, F_{2}\right], F_{i}\right]\right)$ for $i=1$, 2 is non zero at 0 .

It is well-known [33] that there exists a smooth (resp. analytic if $F_{1}, F_{2}$ are analytic) system of coordinates at 0 such that $D$ can be written ker $\omega$, where $\omega$ is Martinet canonical form. Hence $D$ is generated by $F_{1}, F_{2}$ where $F_{1}=\frac{\partial}{\partial x}+\frac{y^{2}}{2} \frac{\partial}{\partial z}, F_{2}=\frac{\partial}{\partial y}$. Consider now a germ of a smooth metric on $D$. It is defined by $g=a(q) d x^{2}+2 b(q) d x d y+c(q) d y^{2}$ where $a, b, c$ are germs at 0 of smooth functions. If $g=d x^{2}+d y^{2}$, the metric is said flat.

LemMa 2.6. Assume that $D=\operatorname{ker} \omega$, where $\omega=d z-\frac{1}{2} y^{2} d x$. Then the set $\Omega: \operatorname{det}\left(F_{1}, F_{2},\left[F_{1}, F_{2}\right]\right)=0$ called the Martinet surface is the plane $y=0$. The abnormal geodesics are the lines $y=0, z=z_{0}$.

Proof. We use proposition 2.4. Abnormal bi-extremals are solutions of

$$
P_{1}=P_{2}=\left\{P_{1}, P_{2}\right\}=0, \sum_{i=1}^{2} u_{i}\left\{\left\{P_{1}, P_{2}\right\}, P_{i}\right\}=0 .
$$

Computing we get

$$
\left[F_{1}, F_{2}\right]=y \frac{\partial}{\partial z},\left[\left[F_{1}, F_{2}\right], F_{1}\right]=0 \quad, \quad\left[\left[F_{1}, F_{2}\right] F_{2}\right]=\frac{\partial}{\partial z} .
$$

Since $p \neq 0$, the abnormal geodesics are contained in the Martinet surface $\operatorname{det}\left(F_{1}, F_{2},\left[F_{1}, F_{2}\right]\right)=0$, that is $y=0$.

The associated controls satisfy

$$
u_{1} D_{1}+u_{2} D_{2}=0 \text {, where } y=0 .
$$

Since $D_{1}=0$ and $D_{2} \neq 0$, we get $u_{2}=0$. Choosing a parametrization we get the curves $\gamma: t \longmapsto\left( \pm t+x_{0}, 0, z_{0}\right)$. Using $P_{1}=P_{2}=0$, the adjoint vector satisfies $p_{x}=p_{y}=0$.

Lemma 2.7. Assume $g=d x^{2}+d y^{2}$. Then the abnormal geodesics are not strictly abnormal.

Proof. The Hamilton function with $\lambda=-\frac{1}{2}$ is:

$$
H_{\lambda}=p_{x} u_{1}+p_{y} u_{2}+p_{z} \frac{y^{2}}{2} u_{1}-\frac{1}{2}\left(u_{1}^{2}+u_{2}^{2}\right) .
$$


Solving $\frac{\partial H_{\lambda}}{\partial u}=0$, we obtain:

$$
u_{2}=p_{y} \quad, \quad u_{1}=p_{x}+p_{z} \frac{y^{2}}{2}
$$

Normal bi-extremals are solutions of:

$$
\begin{array}{ll}
\dot{x}=p_{x}+p_{z} \frac{y^{2}}{2} & \dot{p}_{x}=0 \\
\dot{y}=p_{y} & \dot{p}_{y}=-\left(p_{x}+p_{z} \frac{y^{2}}{2}\right) p_{z} y \\
\dot{z}=\frac{y^{2}}{2}\left(p_{x}+p_{z} \frac{y^{2}}{2}\right) & \dot{p}_{z}=0 .
\end{array}
$$

We check easily that the lines $x= \pm t+x_{0}, y=0, z=z_{0}$ are solutions. Indeed they correspond to $u_{1}= \pm 1, u_{2}=0$. Hence, with $y=0$, we obtain $p_{y}=0, p_{x}= \pm 1$ and $p_{z}$ arbitrary constant. This proves that they are not strictly abnormal.

Definition 2.8. Consider the control systems defined by (2.1) where $U$ is a neighborhood of 0 and the $F_{i}$ 's are germs of smooth (resp. analytic) vector fields on $U$. The set of such control systems can be identified to the set $\mathcal{C}$ of $m$ vector fields $\left(F_{1}, \ldots, F_{m}\right)$. The feedback group $G_{f}$ is the transformation group induced by the following actions on $\mathcal{C}$ :

(i) $Q=\varphi(q)$, where $\varphi$ is a smooth (resp. analytic) germ of diffeomorphism preserving 0 ,

(ii) feedback transformations $v=\theta(q) u$, where $\theta(q) \in G L(m, \mathbb{R})$ and $q \longmapsto \theta(q)$ is a smooth (resp. analytic) germ at 0 .

We can define the action of $G_{f}$ on the set of germs at 0 of Hamiltonian vector fields as follows. The action of a feedback is trivial and a diffeomorphism acts by the canonical symplectic diffeomorphism $\vec{\varphi}$ induced by $\varphi$.

Giving a germ at 0 of a metric $g$ on $D=\operatorname{Span}\left\{F_{1}, \cdots F_{m}\right\}$, we denote by $G$ the subgroup of $G_{f}$ using only feed back transformations preserving the metric $g$.

In invariant theory, covariants are mappings which commute with the respective actions of $G$. Both normal and abnormal bi-extremals provide covariants. First we have

Lemma 2.9. Assume that 0 is a Martinet point. Then the two mappings

$\lambda_{1}: \mathcal{C} \longmapsto$ Martinet surface,

$\lambda_{2}: \mathcal{C} \longmapsto$ unparametrized lines corresponding to abnormal geodesics, are covariants.

Proof. This result can be deduced from [6]. Indeed abnormal geodesics are depending only on $D$. To the control system $(2.1)$, where $m=2$, we can associate two affine control systems by taking $u_{1}=1$ and $u_{2}=1$. This fix locally the parametrization of admissible trajectories. The abnormal geodesics correspond to singular trajectories and $\lambda_{1}, \lambda_{2}$ are covariants for the induced action of the feedback group. Also observe that in the classification of [6], they correspond to exceptional trajectories, because the Hamiltonian is zero. 
LEMMA 2.10. The mapping $\lambda_{3}:(D, g) \longmapsto \vec{H}_{n}$ is a covariant.

Proof. The proof is a straightforward computation. It is simplified if we use orthonormal vector fields. Then a feedback $v=\theta(q) u$ preserving $g$ is such that $\theta(q) \in O(m, \mathbb{R})$, i.e ${ }^{t} \theta \theta=I$.

\subsection{Normal Form: isoperimetric CASE.}

2.3.1. Statement of the problem. When computing a normal form for a pair $(D, g)$ near a Martinet point we have different choices using the previous covariants. We can normalize the distribution $D$ or the metric $g$. We adopt the first choice. Our study is localized near 0 and $D$ is normalized to ker $\omega$, where $\omega=d z-\frac{1}{2} y^{2} d x$. We shall first analyze the isoperimetric case, which is the important case in a gradated normal form and the generalization is straightforward. We use the following relations. The set $\mathcal{G}$ is the set of metrics on $D$ represented as $a(q) d x^{2}+2 b(q) d x d y+c(q) d y^{2}$ where $a, b, c$ are germs at 0 of $C^{\infty}$ functions. The set $\mathcal{G}_{i}$ is the subset of metrics called isoperimetric such that $a, b, c$ are not depending on $z$. We denote by $\mathcal{D}$ the set of germs at 0 of $C^{\infty}$ diffeomorphisms on $\mathbb{R}^{3}$ preserving 0 . The set $\mathcal{D}_{i}$ is the subset of diffeomorphisms $\Phi \in \mathcal{D}:(x, y, z) \longmapsto(X, Y, Z)$, where $X_{z}=Y_{z}=0$. To compute a normal form for $(D, g)$ we use different steps.

Lemma 2.11. Take $\Phi \in \mathcal{D}:(x, y, z) \longmapsto(X, Y, Z)$ such that

(i) $X_{x}(0) \neq 0$

(ii) $d Z-\frac{Y^{2}}{2} d X=d z-\frac{y^{2}}{2} d x$.

Then $\Phi$ is defined by the following equations

$$
Y^{2}=y^{2} \sigma_{X}, \quad x=\sigma+\frac{y}{2} \sigma_{y}, \quad Z=z-\frac{y^{3}}{4} \sigma_{y},
$$

where $\sigma$ is any germ at 0 of a function $(y, X) \longmapsto \mathbb{R}$ such that $\sigma(0)=0$ and $\sigma_{X}(0)>0$.

Proof. By definition

$$
d Z-\frac{Y^{2}}{2} d X=d z-\frac{y^{2}}{2} d x
$$

Hence $Y d X \wedge d Y=y d x \wedge d y$ and $y=0$ is equivalent to $Y=0$.

Since

we get

$$
-\frac{y^{2}}{2} d x=-d\left(\frac{x y^{2}}{2}\right)+x y d y
$$

$$
d\left(Z+\frac{x y^{2}}{2}\right)=\frac{Y^{2}}{2} d X+d z+x y d y .
$$

Assume $X_{x}(0) \neq 0$, hence we can choose $y, z, X$ as coordinates on $\mathbb{R}^{3}$. We introduce the function $S$ defined by

$$
S(y, z, X)=Z+\frac{x y^{2}}{2} .
$$

From (2.5), we obtain

$$
S_{X}=\frac{Y^{2}}{2}, \quad S_{y}=x y, \quad S_{z}=1,
$$


hence $S$ can be written

$$
S=z+\frac{y^{2}}{2} \sigma(y, X),
$$

$\sigma_{X}(0)>0$ and $x, Y$ are defined by the relations:

$$
Y^{2}=y^{2} \sigma_{X}, \quad x=\sigma+\frac{y}{2} \sigma_{y}
$$

and $Z$ is defined by

$$
Z=S-\frac{x y^{2}}{2}
$$

DEFINITION 2.12. Without loosing any generality, we can choose the branch $Y=y \sqrt{\sigma_{X}}$. Therefore the germ $\Phi$ is uniquely defined by the germ $\sigma$ and $\sigma$ is called the generating function of $\Phi$. We denote by $\mathcal{M}$ the set of such germs of diffeomorphism satisfying (i) and (ii).

Proposition 2.13. Let $g \in \mathcal{G}_{i}$ of the form $a(x, y) d x^{2}+2 b(x, y) d x d y+$ $c(x, y) d y^{2}$. If $\Phi:(x, y, z) \mapsto(X, Y, Z)$ is an element of $\mathcal{M}$, whose generating function is $\sigma$, transforming $g$ in a sum of squares $A(X, Y) d X^{2}+$ $C(X, Y) d Y^{2}$, then $\sigma$ is solution of a partial differential equation of the form

$$
3 \sigma_{y}+y \sigma_{y^{2}}=F\left(y, \sigma, \sigma_{X}, y \sigma_{y}, y \sigma_{X X}, y \sigma_{X y}\right)
$$

where $F$ is smooth if $a, b, c$ are smooth and analytic if $a, b, c$ are analytic, near $y=0$.

Proof. We have

$$
x=\sigma+\frac{y}{2} \sigma_{y}, \quad Y=y \sqrt{\sigma_{X}},
$$

then

$$
\begin{aligned}
d x & =\left(\sigma_{X}+\frac{y}{2} \sigma_{X y}\right) d X+\left(\frac{3 \sigma_{y}+y \sigma_{y^{2}}}{2}\right) d y \\
d Y & =\frac{y \sigma_{X X}}{2 \sqrt{\sigma_{X}}} d X+\left(\sqrt{\sigma_{X}}+\frac{y}{2} \frac{\sigma_{X y}}{\sqrt{\sigma_{X}}}\right) d y .
\end{aligned}
$$

We want to transform $g$ into a sum of squares $A(X, Y) d X^{2}+C(X, Y) d Y^{2}$ using a germ $\Phi \in \mathcal{M}$. Since $X, y$ is a system of coordinates, using

$$
\begin{aligned}
A d X^{2} & +C\left(\frac{y}{2} \frac{\sigma_{X X}}{\sqrt{\sigma_{X}}} d X+\left(\sqrt{\sigma_{X}}+\frac{y}{2} \frac{\sigma_{X y}}{\sqrt{\sigma_{X}}}\right) d y\right)^{2} \\
& =a\left[\left(\sigma_{X}+\frac{y}{2} \sigma_{X y}\right) d X+\left(\frac{3 \sigma_{y}+y \sigma_{y^{2}}}{2}\right) d y\right]^{2} \\
& +b\left[\left(2 \sigma_{X}+y \sigma_{X y}\right) d X+\left(3 \sigma_{y}+y \sigma_{y^{2}}\right) d y\right] d y+c d y^{2}
\end{aligned}
$$

we obtain the relations

$$
\begin{aligned}
& A+C \frac{y^{2}}{4 \sigma_{X}} \sigma_{X X}^{2}=a\left(\sigma_{X}+\frac{y}{2} \sigma_{X y}\right)^{2} \\
& \frac{C y \sigma_{X X}}{2 \sigma_{X}}\left(2 \sigma_{X}+y \sigma_{X y}\right)=\frac{a}{2}\left(2 \sigma_{X}+y \sigma_{X y}\right)\left(3 \sigma_{y}+y \sigma_{y^{2}}\right)+b\left(2 \sigma_{X}+y \sigma_{X y}\right) \\
& \frac{C\left(2 \sigma_{X}+y \sigma_{X y}\right)^{2}}{4 \sigma_{X}}=\frac{a}{4}\left(3 \sigma_{y}+y \sigma_{y^{2}}\right)^{2}+b\left(3 \sigma_{y}+y \sigma_{y^{2}}\right)+c . \\
& \text { Esaim: COCV, DeCEMber 1997, Vol. 2, PP. } 377-448
\end{aligned}
$$


By assumption $\sigma_{X}(0)>0$, hence for $y$ small enough, we have $\sigma_{X}+\frac{y}{2} \sigma_{X y} \neq$ 0 . Simplifying in the second equation, we get

$$
\frac{C y \sigma_{X X}}{\sigma_{X}}=a\left(3 \sigma_{y}+y \sigma_{y^{2}}\right)+2 b .
$$

Using this equation $A$ can be computed as

$$
A=a\left(\sigma_{X}+\frac{y}{2} \sigma_{X y}\right)^{2}-\frac{y}{4} \sigma_{X X}\left[a\left(3 \sigma_{y}+y \sigma_{y^{2}}\right)+2 b\right] .
$$

Now, from the third equation $C$ is given by

$$
C=\frac{\sigma_{X}\left[a\left(3 \sigma_{y}+y \sigma_{y^{2}}\right)^{2}+4 b\left(3 \sigma_{y}+y \sigma_{y^{2}}\right)+4 c\right]}{\left(2 \sigma_{X}+y \sigma_{X y}\right)^{2}}
$$

and since $\sigma_{X}(0) \neq 0, \sigma$ is solution for $y$ small enough of the following compatibility equation

$$
\begin{aligned}
& \left(2 \sigma_{X}+y \sigma_{X y}\right)^{2}\left[a\left(3 \sigma_{y}+y \sigma_{y^{2}}\right)+2 b\right] \\
& \quad=y \sigma_{X X}\left[a\left(3 \sigma_{y}+y \sigma_{y^{2}}\right)^{2}+4 b\left(3 \sigma_{y}+y \sigma_{y^{2}}\right)+4 c\right] .
\end{aligned}
$$

This equation can be solved as follows. We set

$$
\lambda=3 \sigma_{y}+y \sigma_{y^{2}} .
$$

Hence $(2.10)$ can be written as

$$
\mathcal{A} \lambda^{2}+\mathcal{B} \lambda+\mathcal{C}=0
$$

where $\mathcal{A}, \mathcal{B}, \mathcal{C}$ are functions of $a, b, c$ and of the jet $\sigma$ defined by

$$
\begin{aligned}
& \mathcal{A}=a y \sigma_{X X} \\
& \mathcal{B}=4 b y \sigma_{X X}-a\left(2 \sigma_{X}+y \sigma_{X y}\right)^{2} \\
& \mathcal{C}=4 c y \sigma_{X X}-2 b\left(2 \sigma_{X}+y \sigma_{X y}\right)^{2} .
\end{aligned}
$$

If $(X, y)=0$, one has $\mathcal{A}=0$ and

$$
\mathcal{B}=-4 a(0) \sigma_{X}^{2}(0), \mathcal{C}=-8 b(0) \sigma_{X}^{2}(0),
$$

where $\sigma_{X}(0)>0$. Since $g>0$, we have $a(0)>0$ and $\mathcal{B} \neq 0$ near 0 . Therefore at $0, \lambda$ solution of (2.11) is given by $\lambda_{0}=-2 b / a$. Now (2.11) can be written as an equation

$$
G(\lambda, J, a, b)=0
$$

where $J$ is a vector in the jet of $\sigma$ and is of the form $\left(\sigma_{X}, y \sigma_{X X}, y \sigma_{X y}\right)$ and we have at $(X, y)=(0,0)$ :

$$
\frac{\partial G}{\partial \lambda}=\mathcal{B}(0) \neq 0
$$

Hence using the implicit function theorem, there exists a smooth (resp. analytic) function $F$ if $a, b, c$ are smooth (resp. analytic) such that

$$
3 \sigma_{y}+y \sigma_{y^{2}}=F\left(y, \sigma, \sigma_{X}, y \sigma_{y}, y \sigma_{X X}, y \sigma_{X y}\right)
$$

for $y$ small enough.

More precisely $F$ can be computed as follows. The discriminant of (2.11) is $\delta=\mathcal{B}^{2}-4 \mathcal{A C}$. Near $y=0, \delta>0$ and the equation has two real roots:

$$
\lambda_{\varepsilon}=\frac{-\mathcal{B}+\varepsilon \mathcal{B} \sqrt{1-4 \mathcal{A C} / \mathcal{B}^{2}}}{2 \mathcal{A}}, \quad \varepsilon= \pm 1
$$


When $y \rightarrow 0, \mathcal{A} \rightarrow 0$ and $\lambda_{\varepsilon}$ behaves like $\frac{-\mathcal{B}+\varepsilon \mathcal{B}\left(1-2 \mathcal{A C} / \mathcal{B}^{2}\right)}{2 \mathcal{A}}$. Hence, when $y \rightarrow 0$, the root corresponding to $\varepsilon=-1$ tends to the infinity and the root corresponding to $\varepsilon=+1$ tends to $-\frac{\mathcal{C}}{\mathcal{B}}=-\frac{2 b}{a}$.

Therefore

$$
3 \sigma_{y}+y \sigma_{y^{2}}=\frac{\mathcal{B}\left(-1+\sqrt{1-4 \mathcal{A C} / \mathcal{B}^{2}}\right)}{2 \mathcal{A}}
$$

where the right side can be expand in a power series of $\left(y, \sigma, \sigma_{X}, y \sigma_{y}\right.$, $\left.y \sigma_{X X}, y \sigma_{X y}\right)$ converging near $\left(0,0, \sigma_{X}(0), 0,0,0\right)$ when $\sigma_{X}(0)>0$.

Proposition 2.14. Consider the Cauchy problem for the singular partial differential equation:

$$
\begin{aligned}
& 3 \sigma_{y}+y \sigma_{y^{2}}=F\left(y, \sigma, \sigma_{X}, y \sigma_{y}, y \sigma_{X X}, y \sigma_{X y}\right) \\
& \sigma(X, y=0)=\sigma_{0}(X) .
\end{aligned}
$$

Let $P=\left(0, \sigma_{0}(0), \sigma_{0}^{\prime}(0), 0,0,0\right)$. If $F$ is smooth at $P$, then there exists an unique power series $\sigma(X, y)=\sum_{n>0} \frac{y^{n}}{n !} \sigma_{n}(X)$ solution of the problem. If $\sigma_{0}$ is analytic at $O$ and $F$ is analytic at $P$, then the power series is converging at 0 . Hence the Cauchy problem admits an unique analytic solution at 0 .

Proof. Our singular particular equation is an equation of high-order BriotBouquet type and we can apply results from section 5 and 6 of [18]. First we compute a formal solution. Indeed consider the equation

$$
3 \sigma_{y}+y \sigma_{y^{2}}=F\left(y, \sigma, \sigma_{X}, y \sigma_{y}, y \sigma_{X X}, y \sigma_{X y}\right)
$$

where $\sigma(X, y)=\sum_{n \geq 0} \frac{\sigma_{n}(X)}{n !} y^{n}$, and $\sigma_{0}(X)$ is given. Evaluating at $y=0$, we get

$$
3 \sigma_{1}=F(\widetilde{P}), \text { where } \widetilde{P}=\left(0, \sigma_{0}, \sigma_{0}^{\prime}, 0,0,0\right) .
$$

Differentiating with respect to $y$ and evaluating at $y=0$ we obtain

$$
4 \sigma_{2}=F_{1}(\widetilde{P})+\left(F_{2}(\widetilde{P})+F_{4}(\widetilde{P})\right) \sigma_{1}+\left(F_{3}(\widetilde{P})+F_{6}(\widetilde{P})\right) \sigma_{1}^{\prime}+F_{5}(\widetilde{P}) \sigma_{0}^{\prime \prime},
$$

where $F_{i}$ is the partial derivative of $F$ with respect to the ith-argument.

Hence, we can compute $\sigma_{2}$. More generally, by successive differentiation, with respect to $y$ and evaluating at $y=0$, we get a recurrence relation

$$
(n+2) \sigma_{n}=\Phi_{n}\left(\sigma_{0}, \sigma_{1}, \ldots, \sigma_{n-1}\right), n \geq 1 .
$$

Moreover the power series $\sigma(X, y)=\sum_{n \geq 0} \frac{\sigma_{n}(X) y^{n}}{n !}$ is such that each $\sigma_{n}$ are analytic in a common disk centered at 0 and $\sigma$ belongs to a Gevrey class $s \geq s_{1}$, where $s_{1}$ is the smallest integer such that the power series

$$
\sum_{n \geq 0} \frac{\sigma_{n}(X) y^{n}}{(n !)^{s_{1}}}
$$

is converging at $(X, y)=(0,0)$.

The integer $s_{1}$ can be easily computed. Indeed we observe that $\sigma_{1}$ is function of $\sigma_{0}, \sigma_{0}^{\prime}, \sigma_{2}$ is function of $\sigma_{0}, \sigma_{0}^{\prime}, \sigma_{0}^{\prime \prime}$ and more generally $\sigma_{n}$ is function of $\sigma_{0}^{(k)}$ for $k \leq n$. This ensures that $s_{1}=1$ as in the CauchyKowaleska theorem and $\sigma$ is analytic at 0 . For instance consider the equation

$$
3 \sigma_{y}+y \sigma_{y^{2}}=y \sigma_{X X}
$$

Esaim: CoCV, December 1997, Vol. 2, PP. 377-448 
we get the recurrence relation

$$
(n+2) \sigma_{n}=(n-1) \sigma_{n-2}^{\prime \prime},
$$

for $n$ even, $n \geq 2$ and $\sigma_{n}(X)=0$ for $n$ odd, $n \geq 1$. Hence for $n$ even we have the estimate

$$
\left|\sigma_{n}(X)\right| \leq\left|\sigma_{0}^{(n)}(X)\right|, \quad n \geq 0
$$

To prove easily the convergence, we use the comparison with the transport equation:

$$
\sigma_{y}=\sigma_{X}
$$

in which we look for a solution $\widetilde{\sigma}(X, y)=\sum_{n>0} \frac{y^{n}}{n !} \widetilde{\sigma_{n}}(X)$, where $\widetilde{\sigma_{0}}(X)=$ $\sigma_{0}(X)$.

The recurrence relation is

$$
\widetilde{\sigma_{n}}(X)=\widetilde{\sigma}_{n-1}^{\prime}(X), \quad n \geq 1
$$

hence

$$
\widetilde{\sigma_{n}}(X)=\sigma_{0}^{(n)}(X)
$$

Using Cauchy estimates for $\sigma_{0}(X)$ or the Cauchy-Kowaleska theorem, we get that $\widetilde{\sigma}(X, y)$ is analytic at 0 . A majorizing function of $\widetilde{\sigma}$ at 0 is also majorizing $\sigma$ and $\sigma$ is analytic at 0 . This example shows how to evaluate the contribution of the different terms of $F$ and also to give a proper weight to each argument, for instance only the terms $\sigma_{X}, y \sigma_{X X}$ and $y \sigma_{X y}$ are providing contributions of $\sigma_{0}^{(n)}(X)$ to $\sigma_{n}(X)$.

LEMMA 2.15. There exists a unique initial function $\sigma_{0}(X)$ satisfying $\sigma_{0}(X)$ $=0, \sigma_{0}^{\prime}(0)>0$ such that $g=A d X^{2}+C d Y^{2}$ can be normalized in such a way that $A$ restricted to $Y=0$ is one. It is solution of the differential equation $\sigma_{0}^{\prime}(X)=\left[a_{0}\left(\sigma_{0}(X)\right)\right]^{-\frac{1}{2}}$ where $a_{0}(x)$ is the restriction of a to $y=0$.

Proof. Recall that relation (2.8) is

$$
A=a\left(\sigma_{X}+\frac{y}{2} \sigma_{X y}\right)^{2}-\frac{y}{4} \sigma_{X X}\left[a\left(3 \sigma_{y}+y \sigma_{y^{2}}\right)+2 b\right] .
$$

Let us set

$$
A(X, Y)=\sum_{n \geq 0} A_{n}(X) \frac{Y^{n}}{n !}
$$

and since $y=0$ is equivalent to $Y=0$, one has $A_{0}(X)=A_{\mid y=0}$.

Using

$$
\sigma(X, y)=\sum_{n \geq 0} \sigma_{n}(X) \frac{y^{n}}{n !}
$$

and

$$
a(x, y)=\sum_{n \geq 0} a_{n}(x) \frac{y^{n}}{n !},
$$

we get from $(2.8)$

$$
A_{0}(X)=a_{0}(x) \sigma_{0}^{2}(X) .
$$

Since for $y=0$, one has $x=\sigma_{0}(X)$, this relation becomes

$$
A_{0}(X)=a_{0}\left(\sigma_{0}(X)\right) \sigma_{0}^{\prime 2}(X) .
$$


Because $g$ is positive near 0 , we have $a_{0}>0$. Hence in order to get the normalisation $A_{0}=1$ with $\sigma_{0}^{\prime}(0)>0$, we must solve the differential equation

$$
\sigma_{0}^{\prime}(X)=a_{0}\left(\sigma_{0}(X)\right)^{-\frac{1}{2}}
$$

It admits near 0 an unique analytic solution such that $\sigma_{0}(0)=0$.

REMARK 2.16. It is important to observe that the diffeomorphism $\Phi \in \mathcal{M}$ reducing $g$ to a sum of squares $A d X^{2}+C d Y^{2}$ where $A(0)=1$ is unique if we choose the branch $Y=y \sqrt{\sigma_{X}}$.

LEMMA 2.17. There exists an unique initial function $\sigma_{0}(X)$ with $\sigma_{0}(0)=0$, $\sigma_{0}^{\prime}(0)>0$ such that the restriction of $C$ to $Y=0$ can be taken equal to one. It is solution of the differential equation

$$
\sigma_{0}^{\prime}(X)=\frac{a_{0}\left(\sigma_{0}(X)\right) c_{0}\left(\sigma_{0}(X)\right)-b_{0}^{2}\left(\sigma_{0}(X)\right)}{a_{0}\left(\sigma_{0}(X)\right)}
$$

where $a_{0}, b_{0}, c_{0}$ designs the respective restrictions of $a, b, c$ to $y=0$.

Proof. From (2.9), we get

$$
C_{\mid y=0}=\frac{a\left(3 \sigma_{y}\right)^{2}+4 b\left(3 \sigma_{y}\right)+4 c}{4 \sigma_{0}^{\prime}}
$$

where for $y=0$, we have from $(2.10)$

$$
3 \sigma_{y}=-2 b / a \text {. }
$$

Hence

$$
C_{\mid y=0}=\frac{a c-b^{2}}{a \sigma_{0}^{\prime}} .
$$

Now if we set $C_{0}(X)=C_{\mid Y=0}=C_{\mid y=0}$, we can impose $C_{0}(X)=1$ if $\sigma_{0}^{\prime}$ is solution of

$$
\sigma_{0}^{\prime}(X)=\frac{a_{0}\left(\sigma_{0}(X)\right) c_{0}\left(\sigma_{0}(X)\right)-b_{0}^{2}\left(\sigma_{0}(X)\right)}{a_{0}\left(\sigma_{0}(X)\right)} .
$$

Since $g>0$ we have $a_{0}>0$ and $a_{0} c_{0}-b_{0}^{2}>0$, at 0 . Hence $\sigma_{0}^{\prime}(0)>0$ and the lemma is proved.

LEMMA 2.18. Let $\Phi:(x, y, z) \longmapsto(X, Y, Z)$ be an analytic element in $\mathcal{D}_{i}$. Assume that $\Phi * \omega=h \omega$, where $h$ is a germ at 0 of a non-vanishing function, $\omega=d z-\frac{1}{2} y^{2} d x$ and $\Phi * \omega$ is the image of $\omega$. Then $h$ is a constant.

Proof. We set

$$
X=\varphi(x, y), \quad Y=\psi(x, y), \quad Z=\theta(x, y, z) .
$$

Writing

$$
\theta_{x} d x+\theta_{y} d y+\theta_{z} d z-\frac{\psi^{2}}{2}\left(\varphi_{x} d x+\varphi_{y} d y\right)=h\left(d z-\frac{y^{2}}{2} d x\right)
$$

we get the following equations

$$
\begin{aligned}
\theta_{x}-\frac{\psi^{2}}{2} \varphi_{x} & =-\frac{h y^{2}}{2} \\
\theta_{y}-\frac{\psi^{2}}{2} \varphi_{y} & =0 \\
\theta_{z} & =h .
\end{aligned}
$$


From (2.14) we can write $\theta_{y}=K(x, y)$, hence we obtain

$$
\theta(x, y, z)=L(x, y)+M(x, z),
$$

and from $(2.15), h=M_{z}$.

Expanding $M$ as $\sum_{n \geq 0} z^{n} M_{n}(x)$ and considering the terms in $z$ in (2.13), we get

$$
\sum_{n \geq 1} z^{n} M_{n}^{\prime}(x)=0
$$

Hence $M_{n}^{\prime}(x)=0$ for $n \geq 1$ and

$$
M(x, z)=M_{0}(x)+\sum_{n \geq 1} z^{n} M_{n},
$$

where $M_{n}$ is a constant for $n \geq 1$. From (2.15), $h=\sum_{n \geq 1} n z^{n-1} M_{n}$ and plugging $h$ in (2.13), we obtain $M_{n}=0$ for $n \geq 2$. Therefore $h$ is a constant.

Theorem 2.19. Consider $(D, g)$, where $D=\operatorname{ker} \omega, \omega=d z-\frac{1}{2} y^{2} d x$ and $g$ is a metric of the form $a(x, y) d x^{2}+2 b(x, y) d x d y+c(x, y) d y^{2}$, where $a, b, c$ are analytic germs a 0 . Then there exists an analytic germ of diffeomorphism $\Phi$ at $0:(x, y, z) \longmapsto(X, Y, Z)$, preserving 0 such that

(i) $X_{x}(0) \neq 0, X_{z}=Y_{z}=0$ and $\Phi$ preserves the distribution ker $\omega$.

(ii) The image of $g$ by $\Phi$ is a sum of squares $A(X, Y) d X^{2}+C(X, Y) d Y^{2}$, where $A(X, Y)=1+Y F(X, Y), C(X, Y)=1+G(X, Y), G(0)=0$.

The mappings $A$ and $C$ are unique if we identify those deduced by a germ of diffeomorphisms from the symmetry group $S$ generated by the two transformations

$$
S_{1}:(X, Y, Z) \longmapsto(-X, Y,-Z), \quad S_{2}:(X, Y, Z) \longmapsto(X,-Y, Z) .
$$

Proof. From (i), $\Phi$ is of the form $X=\varphi(x, y), Y=\psi(x, z)$. From lemma 2.18 , if $\Phi$ preserves ker $\omega$, then $\Phi * \omega=h \omega$, where $h$ is a constant. Therefore all these diffeomorphisms are parametrized by lemma 2.11. Indeed using a transformation of the form

$$
X=\lambda x, \quad Y=y, \quad Z=\lambda z,
$$

where $\lambda$ is a scalar, one may assume $h=1$. Therefore using lemma 2.15 and proposition 2.13, we can assume that the image of $g$ is a sum of squares $A(X, Y)=1+Y F(X, Y), C(X, Y)=C_{0}+G(X, Y)$, where $C_{0}$ is a constant $>0$ and $G(0)=0$.

Endly, we can normalize $C_{0}$ to 1 using a transformation of the form $X^{\prime}=$ $X, Y^{\prime}=\lambda Y, Z^{\prime}=\frac{\lambda^{2}}{2} Z$.

Our work, see in particular the remark 2.16, shows that the coefficients $A$ and $C$ are unique if we identify the metrics deduced using an element of $S$.

\subsection{Geodesics}

Let $\omega=d z-\frac{y^{2}}{2} d x$ and $g=a(x, y) d x^{2}+c(x, y) d y^{2}$. The system is

$$
\dot{q}=u F_{1}+v F_{2}
$$


where

$$
F_{1}=\frac{\partial}{\partial y} \quad, \quad F_{2}=\frac{\partial}{\partial x}+\frac{y^{2}}{2} \frac{\partial}{\partial z}
$$

and

$$
\begin{gathered}
\left(F_{1}, F_{1}\right)=c \quad\left(F_{2}, F_{2}\right)=a \quad, \quad\left(F_{1}, F_{2}\right)=0, \\
(\dot{q}, \dot{q})=u^{2} c+v^{2} a .
\end{gathered}
$$

The hamiltonian for normal bi-extremals is

$$
H_{\lambda}=v\left(p_{x}+p_{z} \frac{y^{2}}{2}\right)+u p_{y}-\frac{1}{2}\left(u^{2} c+v^{2} a\right) .
$$

Hence $\frac{\partial H}{\partial u}=0$ is equivalent to $p_{y}-u c=0$ and $\frac{\partial H}{\partial v}=0$ to $p_{x}+p_{z} \frac{y^{2}}{2}-v a=0$. Therefore the Hamilton function corresponding to normal bi-extremals is then

$$
H_{n}=\frac{1}{2}\left(u^{2} c+v^{2} a\right)=\frac{1}{2}\left[\frac{p_{y}^{2}}{c}+\frac{\left(p_{x}+p_{z} \frac{y^{2}}{2}\right)^{2}}{a}\right] .
$$

Hence normal bi-extremals are solutions of

$$
\begin{aligned}
\dot{x} & =\frac{1}{a}\left(p_{x}+p_{z} \frac{y^{2}}{2}\right) \\
\dot{y} & =\frac{p_{y}}{c} \\
\dot{z} & =\dot{x} \frac{y^{2}}{2} \\
\dot{p}_{x} & =\frac{p_{y}^{2} c_{x}}{2 c^{2}}+\frac{\left(p_{x}+p_{z} \frac{y^{2}}{2}\right)^{2}}{2 a^{2}} a_{x} \\
\dot{p}_{y} & =\frac{p_{y}^{2} c_{y}}{2 c^{2}}+\frac{\left(p_{x}+p_{z} \frac{y^{2}}{2}\right)^{2}}{2 a^{2}} a_{y}-\frac{\left(p_{x}+p_{z} \frac{y^{2}}{2}\right)}{a} y p_{z} \\
\dot{p}_{z} & =0
\end{aligned}
$$

LeMMA 2.20. Assume $g$ in the normal form $a(x, y) d x^{2}+c(x, y) d y^{2}$, where $a(x, y)=1+y F(x, y), c(x, y)=1+G(x, y), G(0)=0$. Then an abnormal geodesic $\gamma: y=0, z=z_{0}$ is not strictly abnormal if and only if the restriction of $F$ to $y \equiv 0$ is identically 0 . In this case the whole trajectory $\gamma$ is an union of conjugate points.

Proof. We may assume the abnormal geodesic parametrized by $x=t, y=0$ and $z=z_{0}$. Assume it is a solution of (2.16). Since $y(t) \equiv 0$, one has $\dot{y}=\frac{p_{y}}{c} \equiv 0$. Hence $p_{y}(t) \equiv 0$. Therefore $\dot{p}_{y} \equiv 0$ and moreover we have $\dot{p}_{y}=\frac{p_{x}^{2} a_{y}}{2 a^{2}}$ for $y=0$ and $p_{y}=0$. Now the control is $\dot{x}=v \equiv 1$ and $u=0$. Therefore $a_{y} \equiv 0$ along $x=t$ and the lemma is proved.

Moreover observe that if $a_{y}=0$ for $y=0$, the choice of $p_{z}$ is arbitrary on each level set $H_{n}=C$. Hence $\gamma$ is formed with conjugate points.

Definition 2.21. In the isoperimetric case, the Riemannian metric $\widetilde{g}=$ $a(x, y) d x^{2}+2 b(x, y) d x d y+c(x, y) d y^{2}$ is called the induced metric. Its geodesics in the normal form are obtained by setting $p_{z}=0$ in equations (2.16) and by projecting the curves on the $(x, y)$ plane. 


\subsection{NORMAL FORM: GENERAL CASE.}

We generalize the computations from section 2.3.

Lemma 2.22. Let $\Phi:(x, y, z) \longmapsto(X, Y, Z)$ be a germ at 0 of a $C^{\infty}$ diffeomorphism such that

(i) $\Phi(0)=0$ and $(y, X, Z)$ is a coordinate system at 0 .

(ii) $\Phi$ preserves the distribution ker $\omega, \omega=d z-\frac{1}{2} y^{2} d x$, that is there exists a smooth germ $h$ such that $h(0) \neq 0$ and $d z-\frac{1}{2} y^{2} d x=h\left(d Z-\frac{1}{2} Y^{2} d X\right)$.

Then there exists two germs at 0 of smooth functions $\alpha: Z \vdash \mathbb{R}, \sigma:$ $(y, X, Z) \longmapsto \mathbb{R}$ such that $\sigma_{X}(0) \alpha_{Z}(0)<0$ and $\Phi$ is defined by the following equations

$$
x=-\left(\sigma+\frac{y}{2} \sigma_{y}\right), \quad Y^{2}=\frac{-y^{2} \sigma_{X}}{\alpha_{Z}+\frac{y^{2}}{2} \sigma_{Z}}, \quad z=\alpha(Z)-\frac{y^{3}}{4} \sigma_{y} .
$$

Proof. By definition

$$
d\left(z-\frac{y^{2}}{2} x\right)+x y d y=h d Z-h \frac{Y^{2}}{2} d X .
$$

Let us introduce the generating function $S$ of $\Phi$ defined by

$$
S(y, X, Z)=z-\frac{y^{2}}{2} x,
$$

where $(y, X, Z)$ are coordinates. Hence we have

$$
S_{y} d y+S_{X} d X+S_{Z} d Z=h d Z-h \frac{Y^{2}}{2} d X-x y d y .
$$

To compute $S$ we must solve the following equations

$$
\begin{aligned}
x y & =-S_{y} \\
h \frac{Y^{2}}{2} & =-S_{X} \\
h & =S_{Z} .
\end{aligned}
$$

If we write

$$
S=\alpha(X, Z)+y \beta(X, Z)+\frac{y^{2}}{2} \sigma(y, X, Z)
$$

and plugging $y=0$ into (2.17), we get $\beta=0$. Hence

$$
S=\alpha(X, Z)+\frac{y^{2}}{2} \sigma(y, X, Z) .
$$

Solving (2.17), we obtain

$$
x=-\frac{1}{y} S_{y}=-\left(\sigma+\frac{y}{2} \sigma_{y}\right) .
$$

Now, in order to simplify our computations, we shall use that $\Phi$ has the following geometry property.

(P) It has to apply $y=0$ onto $Y=0$. Indeed those sets represent the Martinet surface in the respective coordinates. This surface is filled with the abnormal geodesics. More precisely $\Phi$ must transform each (unparametrized) abnormal geodesic into an abnormal geodesic. Hence it has to apply a line $y=0, z=z_{0}$ onto a line $Y=0, Z=Z_{0}$.

Esaim: CoCv, December 1997, Vol. 2, PP. $377-448$ 
Using (2.18), with $Y=0$, we get $S_{X}=0$ when $Y=0$, i.e,

$$
\alpha_{X}+\frac{y^{2}}{2} \sigma_{X}=0 \text { when } y=0 \text {. }
$$

Therefore $\alpha_{X}=0$ and the generating function can be written

$$
S=\alpha(Z)+\frac{y^{2}}{2} \sigma(y, X, Z)
$$

Using (2.18), (2.19) we get

$$
Y^{2}=-\frac{y^{2} \sigma_{X}}{\alpha_{Z}+\frac{y^{2}}{2} \sigma_{Z}} .
$$

In particular, we must have

$$
\sigma_{X}(0) \alpha_{Z}(0)<0
$$

and

$$
Y= \pm y \sqrt{-\frac{\sigma_{X}}{\alpha_{Z}+\frac{y^{2}}{2} \sigma_{Z}}}
$$

Endly

$$
z=S+\frac{y^{2}}{2} x=\alpha(Z)-\frac{y^{3}}{4} \sigma_{y}
$$

and the lemma is proved.

Theorem 2.23. Assume $g \in \mathcal{G}, g=a d x^{2}+2 b d x d y+c d y^{2}$ and $a, b, c$ are germs at 0 of analytic functions. Then there exists a germ at 0 of an analytic diffeomorphism $\Phi:(x, y, z) \longmapsto(X, Y, Z)$ such that

(i) $\Phi(0)=0$ and $d Z-\frac{1}{2} Y^{2} d X=h\left(d z-\frac{1}{2} y^{2} d x\right), h(0) \neq 0$.

(ii) The image of $g$ by $\Phi$ is given by a sum of squares $A(X, Y, Z) d X^{2}+$ $C(X, Y, Z) d Y^{2}$ where $A=1+Y F(X, Y, Z), C=1+G(X, Y, Z), G_{\left.\right|_{X=Y=0}}=$ 0 .

Proof. We shall transform $g=a d x^{2}+2 b d x d y+c d y^{2}$ into a sum of squares $A d X^{2}+C d Y^{2}$ using a diffeomorphism $\Phi$ where

$$
\begin{aligned}
x & =-\left(\sigma+\frac{y}{2} \sigma_{y}\right) \\
Y & =y\left(-\frac{\sigma_{X}}{\alpha_{Z}+\frac{y^{2}}{2} \sigma_{Z}}\right)^{\frac{1}{2}} .
\end{aligned}
$$

Now, observe that on $D$, we have

$$
d Z=\frac{Y^{2}}{2} d X=\frac{y^{2}}{2}\left(-\frac{\sigma_{X}}{\alpha_{Z}+\frac{y^{2}}{2} \sigma_{Z}}\right) d X .
$$

Hence, computing we get

$$
d x=\varepsilon d X+\beta d y \text { on } D,
$$

where

$$
\begin{aligned}
\varepsilon & =-\left(\sigma_{X}+\frac{y}{2} \sigma_{X y}\right)+\frac{y^{2}}{2}\left(\sigma_{Z}+\frac{y}{2} \sigma_{Z y}\right)\left(\frac{\sigma_{X}}{\alpha_{Z}+\frac{y^{2}}{2} \sigma_{Z}}\right) \\
\beta & =-\left(\frac{3 \sigma_{y}+y \sigma_{y^{2}}}{2}\right),
\end{aligned}
$$


and similarly

$$
d Y=\gamma d X+\delta d y \text { on } D
$$

where

$$
\begin{aligned}
\gamma= & \frac{y}{2}\left(-\frac{\sigma_{X}}{\alpha_{Z}+\frac{y^{2}}{2} \sigma_{Z}}\right)^{-\frac{1}{2}}\left[\frac{\frac{y^{2}}{2} \sigma_{X} \sigma_{X Z}-\sigma_{X^{2}}\left(\alpha_{Z}+\frac{y^{2}}{2} \sigma_{Z}\right)}{\left(\alpha_{Z}+\frac{y^{2}}{2} \sigma_{Z}\right)^{2}}\right] \\
& +\frac{y^{3}}{4}\left(-\frac{\sigma_{X}}{\alpha_{Z}+\frac{y^{2}}{2} \sigma_{Z}}\right)^{\frac{1}{2}}\left[\frac{\alpha_{Z^{2}} \sigma_{X}+\frac{y^{2}}{2} \sigma_{X} \sigma_{Z^{2}}-\sigma_{X Z}\left(\alpha_{Z}+\frac{y^{2}}{2} \sigma_{Z}\right)}{\left(\alpha_{Z}+\frac{y^{2}}{2} \sigma_{Z}\right)^{2}}\right] \\
\delta= & \left(-\frac{\sigma_{X}}{\alpha_{Z}+\frac{y^{2}}{2} \sigma_{Z}}\right)^{\frac{1}{2}} \\
& +\frac{y}{2}\left(-\frac{\sigma_{X}}{\alpha_{Z}+\frac{y^{2}}{2} \sigma_{Z}}\right)^{-\frac{1}{2}}\left[\frac{\frac{y^{2}}{2} \sigma_{X} \sigma_{y Z}+y \sigma_{X} \sigma_{Z}-\sigma_{X y}\left(\alpha_{Z}+\frac{y^{2}}{2} \sigma_{Z}\right)}{\left(\alpha_{Z}+\frac{y^{2}}{2} \sigma_{Z}\right)^{2}}\right] .
\end{aligned}
$$

Hence if we write

$g=A d X^{2}+C(\gamma d X+\delta d y)^{2}=a(\varepsilon d X+\beta d y)^{2}+2 b(\varepsilon d X+\beta d y) d y+c d y^{2}$, we get the relations

$$
\begin{aligned}
A+C \gamma^{2} & =a \varepsilon^{2} \\
C \gamma \delta & =\varepsilon(a \beta+b) \\
C \delta^{2} & =\left(a \beta^{2}+2 b \beta+c\right) .
\end{aligned}
$$

For any $y=0, \varepsilon=-\sigma_{X}, \delta=\sqrt{-\sigma_{X} / \alpha_{Z}}$, hence $\varepsilon$ and $\delta$ are $\neq 0$ at 0 . As in the proof of proposition 2.13 , we get the compatibility relation

$$
\gamma\left(a \beta^{2}+2 b \beta+c\right)=\varepsilon \delta(a \beta+b) .
$$

This equation is solved as in 2.13 , with respect to $-2 \beta=3 \sigma_{y}+y \sigma_{y^{2}}$ and we get an equation of the form

$3 \sigma_{y}+y \sigma_{y^{2}}=F\left(y, \alpha_{Z}, \alpha_{Z^{2}}, \sigma, \sigma_{X}, y \sigma_{Z}, y \sigma_{y}, y \sigma_{X^{2}}, y \sigma_{Z^{2}}, y \sigma_{X y}, y \sigma_{X Z}, y \sigma_{Z y}\right)$, where $\sigma_{X}(0) \alpha_{Z}(0)<0$ and $F$ is analytic at $P=\left(0, \alpha_{Z}(0), 0, \sigma_{X}(0), 0,0\right.$, $0,0,0,0,0)$.

This equation is solved as a Cauchy problem, depending upon an initial function: $\sigma_{0}(X, Z)=\sigma_{\mid y=0}$

$$
\begin{gathered}
3 \sigma_{y}+y \sigma_{y^{2}}=F \\
\sigma_{\mid y=0}=\sigma_{0}(X, Z) .
\end{gathered}
$$

As in section 2.3, this equation admits an unique converging power series at 0 . Hence $g$ can be written as a sum of squares. To get the additional normalisations $A_{\mid Y=0}=1$ and $C_{\mid X=Y=0}=1$, we must solve

$$
\begin{gathered}
\sigma_{X}=a_{0}^{-\frac{1}{2}}(W) \\
\alpha_{Z}=-\left(a_{0}\left(W_{0}\right) c_{0}\left(W_{0}\right)-b_{0}^{2}\left(W_{0}\right)\right)^{-1} a_{0}^{\frac{1}{2}}\left(W_{0}\right),
\end{gathered}
$$

where $W=(-\sigma(X, Z), \alpha(Z)), W_{0}=(0, \alpha(Z))$ and $a_{0}, b_{0}, c_{0}$ are the respective restrictions of $a, b, c$ to $y=0$. The appropriated boundary conditions are

$$
\sigma(0, Z)=0, \quad \alpha(0)=0
$$


and the equations can be solved in cascade.

The theorem is then proved.

2.5.1. Gradated normal Forms. We use the following gradations for the variables. The weight of $x, y$ is 1 , the weight of $z$ is 3 and the corresponding weights for vector fields are -1 for $\frac{\partial}{\partial x}, \frac{\partial}{\partial y}$ and -3 for $\frac{\partial}{\partial z}$. In the normal form, the distribution ker $\omega$ is generated by

$$
F_{1}=\frac{\partial}{\partial x}+\frac{y^{2}}{2} \frac{\partial}{\partial z}, F_{2}=\frac{\partial}{\partial y}
$$

and if the metric is $g=a d x^{2}+c d y^{2}$, we define two orthonormal vector fields by setting $\widetilde{F}_{1}=F_{1} / \sqrt{a}, \widetilde{F}_{2}=F_{2} / \sqrt{c}$. We obtain a normal form of order $p \geq-1$ by expanding $1 / \sqrt{a}, 1 / \sqrt{c}$ in power series at 0 and keeping the terms of order $\leq p$. The associated truncated vector fields are denoted $G_{p}$, $H_{p}$. We get

* order -1 :

$$
\begin{aligned}
& G_{-1}=\frac{\partial}{\partial x}+\frac{y^{2}}{2} \frac{\partial}{\partial z} \\
& H_{-1}=\frac{\partial}{\partial y} .
\end{aligned}
$$

It corresponds to the flat case: $g=d x^{2}+d y^{2}$.

* order 0:

$$
\begin{aligned}
& G_{0}=(1+\alpha y)\left(\frac{\partial}{\partial x}+\frac{y^{2}}{2} \frac{\partial}{\partial z}\right) \\
& H_{0}=(1+\beta x+\gamma y) \frac{\partial}{\partial y},
\end{aligned}
$$

where $\alpha, \beta, \gamma$ are arbitrary constants.

It can be used as a model to study the generic situation. Observe also that the $z$ variable appears in the power series only when $p \geq 2$. Hence for the gradated normal form of order $p$, we are in the isoperimetric case when $p \leq 2$.

\section{TWO GEOMETRIC PROPERTIES OF JACOBI EQUATION}

\subsection{JACOBI EQUATION}

Definition 3.1. Let $M$ be a smooth $n$-dimensional manifold. If $x$ is a coordinate system on $M$, we shall denote by $(x, p)$ the associated coordinate system on the cotangent space $T^{*} M$. The tangent space of $T^{*} M$ splits into the horizontal space $h$ which are vectors of the form $a \frac{\partial}{\partial x}$ and the vertical space $v$ which are vectors of the form $b \frac{\partial}{\partial p}$. Similarly the cotangent space splits into horizontal and vertical forms. For instance the Liouville form $\alpha=p d x$ is horizontal. We shall denote $\pi_{h}$ and $\pi_{u}$ the respective projections from $T\left(T^{*} M\right)$ onto $h$ and $v$.

Definition 3.2. Let $H$ be a smooth function on $T^{*} M$ and let $t \rightarrow \gamma(t), t \in$ $[0, T]$ be a curve solution of the Hamiltonian vector field $\vec{H}$. The variational equation on $T\left(T^{*} M\right)$ from $\vec{H}$ along $\gamma$ will be called the Jacobi equation. A Jacobi field $J:[0, T] \longmapsto T\left(T^{*} M\right)$ is a solution of the Jacobi equation. We shall denote by $E$ the $n$-dimensional vector space generated by Jacobi fields such that $J(0)$ is vertical. Let $\delta: \lambda \longmapsto \delta(\lambda)$ be a $C^{1}$-curve on $T^{*} M$ starting 
at $\lambda=0$ from $\gamma(0)$. A Jacobi field is given by $J(t)=d(\exp t \vec{H})(\dot{\delta}(0))$, where $\dot{\delta}(0)=J(0)$ and $\exp t \vec{H}$ denotes the one-parameter group generated by $\vec{H}$.

Assumption 3.3. From now on we shall assume that the mapping $p \longmapsto$ $H(x, p)$ is a quadratic form. In particular if $C \neq 0$, the level set $H=C$ is a smooth submanifold. Moreover assume $\gamma=(x, p), \dot{x}(t) \neq 0$.

LEMMA 3.4. Let $x\left(t, x_{0}, p_{0}\right), p\left(t, x_{0}, p_{0}\right)$ be the solution of $\vec{H}$ starting from $\left(x_{0}, p_{0}\right)$ at $t=0$. Then for $\lambda \in \mathbb{R}^{*}$ we have

(i) $x\left(t, x_{0}, \lambda p_{0}\right)=x\left(\lambda t, x_{0}, p_{0}\right)$;

(ii) $p\left(t, x_{0}, \lambda p_{0}\right)=\lambda p\left(\lambda t, x_{0}, p_{0}\right)$.

LEMMA 3.5. Consider the curve on $T^{*} M$ starting from $\left(x_{0}, p_{0}\right), p_{0} \neq 0$ and defined by $\delta(\lambda)=\left(x_{0}, p_{0}+\lambda p_{0}\right)$. Then $\dot{\delta}(0)=p_{0} \frac{\partial}{\partial p}$ is vertical. Let $J_{1}$ be the Jacobi field such that $J_{1}(0)=\dot{\delta}(0)$. Then $\pi_{h}\left(J_{1}(t)\right)=t \dot{x}(t)$ where $\gamma=(x, p)$ is the reference trajectory.

Proof. Use lemma 3.4 and the definition of a Jacobi field.

Definition 3.6. Assume that $\gamma=(x, p)$ is a normal bi-extremal defined on $[0, T]$. The time $0<t_{c} \leq T$ is said conjugate to 0 , along $\gamma$ if there exists a non trivial Jacobi field $J$ such that $J(0)$ and $J\left(t_{c}\right)$ are vertical and the point $x\left(t_{c}\right)$ is said conjugate to $x(0)=x_{0}$ along $\gamma$. The set of such fields span a vector space whose dimension is called the index of the conjugate point. One shall denote by $t_{1 c}$ the first conjugate time. If we fix $x_{0}$, the set of first conjugate points $x\left(t_{1 c}\right)$, is the conjugate locus $C\left(x_{0}\right)$. It is a standard fact that this definition of the conjugate locus is equivalent to the one given in the introduction.

LEMMA 3.7. Let $v$ be the vertical space at $\left(x_{0}, p_{0}\right), p_{0} \neq 0$ identified to $\mathbb{R}^{n}$ and let $v^{\prime}$ be any subspace such that $v=\mathbb{R} p_{0} \oplus v^{\prime}$. Let $e_{2}, \ldots, e_{n}$ be a basis of $v^{\prime}$ and let us denote $J_{2}, \ldots, J_{n}$ the Jacobi fields such that $J_{i}(0)=e_{i}$ for $i=2, \ldots, n$. At $\gamma(t)=(x(t), p(t)), \dot{x}(t) \neq 0$, let $h^{\prime}$ be any subspace such that $h=\mathbb{R} \dot{x}(t) \oplus h^{\prime}$ and let $\pi_{h^{\prime}}$ be the projection from $T\left(T^{*} M\right)$ onto $h^{\prime}$. The conjugate time $t_{1 c}$ is the first $0<t \leq T$ such that $\operatorname{det}\left(\pi_{h^{\prime}}\left(J_{2}(t), \ldots, J_{n}(t)\right)\right)=$ 0 .

Proof. Let $J$ be a Jacobi field such that

$$
J(0)=\sum_{i=1}^{n} \lambda_{i} J_{i}(0), \quad\left(\lambda_{1}, \ldots, \lambda_{n}\right) \neq 0 .
$$

Then

$$
\begin{aligned}
\pi_{h}(J(t)) & =\sum_{i=1}^{n} \lambda_{i} \pi_{h}\left(J_{i}(t)\right) \\
& =\left(t \lambda_{1}+\sum_{i=2}^{n} a_{i}(t) \lambda_{i}\right) \dot{x}(t)+\sum_{i=2}^{n} \lambda_{i} \pi_{h^{\prime}}\left(J_{i}(t)\right) .
\end{aligned}
$$


Therefore at $t=t_{1 c}$, we must have a non trivial solution to the equations

$$
\begin{aligned}
t \lambda_{1}+\sum_{i=2}^{n} a_{i}(t) \lambda_{i} & =0 \\
\sum_{i=2}^{n} \lambda_{i} \pi_{h^{\prime}}\left(J_{i}(t)\right) & =0 .
\end{aligned}
$$

Hence a necessary and sufficient condition is

$$
\operatorname{det}\left(\pi_{h^{\prime}}\left(J_{2}(t), \ldots, J_{n}(t)\right)\right)=0
$$

at $t=t_{1 c}$.

REMARK 3.8. This is some freedom in the choice of the complementary space $v^{\prime}$ and one choice is canonical in the sense that it will simplify the effective computation of the conjugate locus. This simplification is well known in Riemannian geometry [15] and was used in [17] in sub-Riemannian geometry. It is a consequence of the following lemma.

LEMMA 3.9. [17] The one-parameter group $\exp t \vec{H}$ preserves the restriction of the Liouville form $\alpha$ on the level set $H=\frac{1}{2}$.

Corollary 3.10. Let $v_{c}$ be the $(n-1)$ dimensional space $v \cap T\left(H=\frac{1}{2}\right)$ at $\left(x_{0}, p_{0}\right)$ chosen in $H=\frac{1}{2}$. Let $\epsilon_{2}, \ldots, \epsilon_{n}$ be a basis of $v_{c}$ and $J_{2}, \ldots, J_{n}$ be the Jacobi fields defined by $J_{i}(0)=\epsilon_{i}$ for $i=2, \ldots, n$. Then $\alpha\left(d \exp t \vec{H}\left(J_{i}(0)\right)=\right.$ 0 for $i=2, \ldots, n$.

Proof. First observe that the curve $\delta(\lambda)=\left(x_{0}, p_{0}+\lambda p_{0}\right)$ is transverse to $H=$ $\frac{1}{2}$, at $\lambda=0$, hence $\dot{\delta}(0)=J_{1}(0) \notin T\left(H=\frac{1}{2}\right)$. Now by construction, since $J_{i}(0)$ is vertical and the Liouville form $\alpha$ is horizontal, we have $\alpha\left(J_{i}(0)\right)=0$ for $i=1, \ldots, n$. Since $J_{i}(t)$ is tangent to $H=\frac{1}{2}$ by construction for $i=2, \ldots, n$, using lemma 3.9, we get $\alpha\left(J_{i}(t)\right)=\alpha\left(J_{i}(0)\right)=0$, for $i=2, \ldots, n$.

Corollary 3.11. Let us choose $(n-1)$ horizontal forms $\alpha_{2}, \ldots, \alpha_{n}$ such that $\alpha \wedge \alpha_{2} \wedge \cdots \wedge \alpha_{n} \neq 0$, along the bi-extremal $\gamma$. Then $t_{c}$ is conjugate to 0 if and only if $\operatorname{det}\left(\alpha_{i}\left(J_{j}(t)\right)=0, i, j=2, \ldots, n\right.$ for $t=t_{c}$.

3.1.1. Application to the Martinet flat case. We consider the equations satisfied by normal bi-extremals in Martinet flat case, using the notations $\omega=d z-\frac{1}{2} y^{2} d x, g=d x^{2}+d y^{2}$.

$$
\begin{aligned}
\dot{x} & =F & \dot{p}_{x} & =0 \\
\dot{y} & =p_{y} & \dot{p}_{y} & =-y p_{z} F \\
\dot{z} & =\frac{y^{2}}{2} F & \dot{p}_{z} & =0,
\end{aligned}
$$

where $p_{x}=\eta, p_{z}=\xi, \eta, \xi$ being constant and $F$ is the function $p_{x}+p_{z} \frac{y^{2}}{2}=$ $\eta+\xi \frac{y^{2}}{2}$. The Hamiltonian is

$$
H_{n}=\frac{1}{2}\left(p_{y}^{2}+F^{2}(y, \eta, \xi)\right)
$$

and is constant along a bi-extremal. The set $H_{n}=\frac{1}{2}$ corresponds to biextremals parametrized by arc-length. 
The variational equation is

$$
\begin{aligned}
\dot{\delta x} & =F_{y} \delta y+F_{\eta} \delta \eta+F_{\xi} \delta \xi \\
\dot{\delta y} & =\delta p_{y} \\
\dot{\delta} z & =y F \delta y+\frac{y^{2}}{2}\left(F_{y} \delta y+F_{\eta} \delta \eta+F_{\xi} \delta \xi\right) \\
\dot{\delta p_{y}} & =-\delta y\left(y F_{y} \xi+\xi F\right)-y \xi F_{\eta} \delta \eta-y \delta \xi\left(\xi F_{\xi}+F\right) \\
\dot{\delta p_{x}} & =\dot{\delta p_{z}}=0 .
\end{aligned}
$$

It corresponds to a time-depending linear Hamiltonian differential equation whose Hamiltonian is a quadratic mapping with respect to $\left(\delta x, \delta y, \delta z, \delta p_{x}\right.$, $\left.\delta p_{y}, \delta p_{z}\right)$.

The computations of conjugate points are highly simplified if we use the corollary 3.11 with the two forms

$$
\alpha_{2}=d x \quad, \quad \alpha_{3}=d y .
$$

Indeed, since

$$
\alpha=p_{x} d x+p_{y} d y+p_{z} d z
$$

the forms $\alpha, \alpha_{2}, \alpha_{3}$ are independant if and only if $p_{z} \neq 0$. Now observe the following. By setting $p_{z}=0$ in (3.2), the projections of the bi-extremals in the $(x, y)$ plane are precisely the geodesics for the Riemannian metric $\widetilde{g}$ induced by the metric $g$ in the plane $(x, y)$. They project onto lines $x=$ $p_{x} t+x_{0}, y=p_{y} t+y_{0}$ and correspond to minimizers for the sub-Riemannian metric because the length of a curve is the euclidian length of its projection on the plane $(x, y)$. Hence according to the general theory, see [5], [19], a strictly normal geodesic cannot be a minimizer beyond its first conjugate point. Therefore such bi-extremals are without conjugate point and we may assume in our study $p_{z} \neq 0$.

In order to compute the conjugate locus from the Martinet point 0 , we must find a basis $e_{2}, e_{3}$ of the intersection $T\left(H=\frac{1}{2}\right)$ with the vertical space: $\delta x=\delta y=\delta z=0$. Computing we get

$$
p_{x} \delta p_{x}+p_{y} \delta p_{y}=0
$$

where $p_{x}^{2}+p_{y}^{2}=1$. We choose $\epsilon_{2}=\left(\delta p_{x}, \delta p_{y}, 0\right)$ non zero solution of $(3.4)$ and $e_{3}=(0,0,1)$. Let $J_{2}, J_{3}$ be the Jacobi fields such that $J_{2}(0)=\epsilon_{2}$ and $J_{3}(0)=e_{3}$. If we set for $i=2,3$,

$$
\pi_{h}\left(J_{i}(t)\right)=\left(\delta_{i} x(t), \delta_{i} y(t), \delta_{i} z(t)\right),
$$

then the conjugate times $t_{c}$ are solutions of

$$
\delta_{2} x(t) \delta_{3} y(t)-\delta_{3} x(t) \delta_{2} y(t)=0 .
$$

It is equivalent to the following computation. Consider the cylinder $C$ : $p_{x}^{2}+p_{y}^{2}(0)=1, p_{z}(0)=\lambda$, which can be parametrized by $(\varphi, \lambda)$ where $\varphi$ is the angle defined by $p_{x}(0)=\sin \varphi, p_{y}(0)=\cos \varphi$. Now, integrate (3.2) from $x=y=z=0$. This defines the exponential mapping

$$
\exp _{0}:(\varphi, \lambda, t) \longmapsto(x(t, \varphi, \lambda), y(t, \varphi, \lambda), z(t, \varphi, \lambda)) .
$$

Equation (3.5) is equivalent to the following equation

$$
\frac{\partial x}{\partial \varphi} \frac{\partial y}{\partial \lambda}-\frac{\partial x}{\partial \lambda} \frac{\partial y}{\partial \varphi}=0 .
$$


Observe that the evaluation of $C(0)$ doesn't require the computation of $z(t, \varphi, \lambda)$. This will save a lot a computations later. This result can be extended locally to the isoperimetric situation.

Proposition 3.12. Consider an isoperimetric metric $g$ and let $H_{n}$ be the Hamilton function corresponding to normal bi-extremals. Let $(\varphi, \lambda)$ be any parametrization of the cylinder $C$ defined by $H_{n}\left(q_{0}, p_{0}\right)=\frac{1}{2}$, where $q_{0}=0$ and let $x(t, \varphi, \lambda), y(t, \varphi, \lambda)$ be the projections of $\exp _{0}$ onto the plane $(x, y)$. Then there exists a neighborhood $U$ of 0 such that the conjugate points associated to strictly normal geodesics and contained in $U$ are obtained by solving the equation

$$
\frac{\partial x}{\partial \varphi} \frac{\partial y}{\partial \lambda}-\frac{\partial x}{\partial \lambda} \frac{\partial y}{\partial \varphi}=0
$$

Proof. It is sufficient to observe that there exists a neighborhood of 0 such that the geodesics where $p_{z}$ vanishes are without conjugate points if there are strictly normal. In the isoperimetric case $H_{n}$ is not depending upon $z$ and $p_{z}$ is constant along a bi-extremals. Geodesics corresponding to $p_{z}=0$ project on the $(x, y)$ plane onto geodesics corresponding to the induced Riemannian metric $\widetilde{g}$. According to classical theory [15], there exists a neighborhood $\widetilde{U}$ of 0 in the $(x, y)$ plane such that geodesics starting from 0 are minimizers on $\widetilde{U}$ for the metric $\widetilde{g}$. Hence they correspond to minimizing curves for the sub-Riemannian metric $g$ and are without conjugate points since according to a well known result [15], strictly normal geodesics cease to be minimizing beyond the first conjugate point. If we take $U=\widetilde{U} \times \mathbb{R}$, the proposition is proved.

\subsection{HaMiLTONIAN VARIATIONAL EQUATION}

Definition 3.13. Let $x=\left(x^{1}, \ldots, x^{n}\right), p=\left(p_{1}, \ldots, p_{n}\right)$ and $K$ be a smooth function on $T^{*} M \times \mathbb{R}$. The coordinate $x^{1}$ is called cyclic if $K$ is not depending upon $x^{1}$. The Hamiltonian vector field $\vec{K}$ is said completely integrable if the set of solutions can be computed by quadratures.

The proofs of the following two lemmas are straightforward.

LEMMA 3.14. If $x^{2}, \ldots, x^{n}$ are cyclic coordinates, then $p_{2}, \ldots, p_{n}$ are first integrals. Moreover if $K$ is not depending on $t$, the Hamiltonian vector field $\vec{K}$ is completely integrable.

LemMa 3.15. Assume that $x^{1}$ is a cyclic coordinate for $H$, then $\delta x^{1}$ is a cyclic coordinate for the Hamiltonian variational equation $\widetilde{H}$ and $\delta p_{1}$ is a first integral.

Proposition 3.16. Assume that the Hamilton function is of the form $\frac{1}{2} a\left(x^{1}\right) p_{1}^{2}+b\left(x^{1}, p_{2}, \ldots, p_{n}\right)$ where $a$ is a non-vanishing function. Then the variational equation whose Hamiltonian is $\widetilde{H}$ is completely integrable.

Proof. The proof is not straightforward because $\widetilde{H}$ is time depending and we have $\frac{d \widetilde{H}}{d t}=\frac{\partial \widetilde{H}}{\partial t}$, along a trajectory $t \longmapsto(\delta x(t), \delta p(t))$. Hence $\widetilde{H}$ is not in general a first integral. 
To prove the result we proceed as follows. First from lemma $3.14, p_{2}, \ldots, p_{n}$ are first integrals and if we set $\eta=\left(p_{2}, \ldots, p_{n}\right), \eta$ will be a constant vector along a trajectory. In order to simplify the notations, we let $x=x^{1}, p=p_{1}$ and the Hamiltonian can be written as

$$
H(x, p, \eta)=\frac{1}{2} a(x) p^{2}+b(x, \eta) .
$$

Hence the solutions of $\vec{H}$ are satisfying

$$
\dot{x}=a p, \quad \dot{p}=-\left(\frac{1}{2} a^{\prime} p^{2}+b^{\prime}\right),
$$

where $a^{\prime}, b^{\prime}$ are the derivatives with respect to $x$. The variational equation is defined by

$$
\begin{aligned}
\dot{\delta x} & =a^{\prime} p \delta x+a \delta p \\
\dot{\delta p} & =-\left(\frac{1}{2} a^{\prime \prime} p^{2} \delta x+b^{\prime \prime} \delta x+a^{\prime} p \delta p\right)+G
\end{aligned}
$$

where $G$ is a function of $\delta \eta$. These two equations can be written as an unique second order differential equation. Indeed we differentiate the first equation with respect to $t$ and we express in this equation $\delta p$ and $\dot{\delta p}$ with respect to $\delta x$ et $\dot{\delta} x$. Computing we obtain the equation

$$
\ddot{\delta} x+A \dot{\delta} x+B \dot{\delta} x=I
$$

where $A=-a^{\prime} p, B=\frac{1}{2} a^{\prime 2} p^{2}+a^{\prime} b^{\prime}+a b^{\prime \prime}-\frac{1}{2} a a^{\prime \prime} p^{2}$ and $I$ is a function of $\delta \eta$.

Moreover we have

$$
\begin{aligned}
& \ddot{x}=\frac{1}{2} a a^{\prime} p^{2}-a b^{\prime} \\
& \ddot{x}=\frac{1}{2} a^{2} a^{\prime \prime} p^{3}-2 a a^{\prime} b^{\prime} p-a^{2} b^{\prime \prime} p
\end{aligned}
$$

and we check that $\dot{x}$ is solution of the equation (3.7) in which the right side is zero. This implies that (3.7) can be integrated by quadratures and the proposition is proved.

3.2.1. Application to the Martinet flat case. We shall detail all the computations concerning the equation (3.3). The coordinates $\delta x$ and $\delta z$ are cyclic coordinates and $\delta p_{x}, \delta p_{z}$ are the corresponding first integrals. Computing we get that $\delta y$ is solution of the second-order differential equation

$$
\ddot{\delta} y+a \delta y=b
$$

where

$$
a=\xi\left(F+y F_{y}\right)
$$

and

$$
b=-y \xi F_{\eta} \delta \eta-y\left(\xi F_{\xi}+F\right) \delta \xi .
$$

Since $\dot{y}$ is solution of (3.8) with 0 right side, the solution of this equation can be written as $\delta y=\dot{y} \psi$, where $\psi$ is a meromorphic function which can be computed using two quadratures. Indeed by plugging $\delta y$ in (3.8) we get

$$
\psi(\dddot{y}+a \dot{y})+\ddot{\psi} \dot{y}+2 \ddot{y} \dot{\psi}=b,
$$

and using $\dddot{y}+a \dot{y}=0$, we obtain

$$
\ddot{\psi} \dot{y}+2 \ddot{y} \dot{\psi}=b .
$$


Hence

$$
d\left(\dot{\psi} \dot{y}^{2}\right)=b \dot{y} d t
$$

and we get

$$
\psi(t)=\psi(0)+\int_{0}^{t} \frac{d s}{\dot{y}^{2}(s)}\left(C+\int_{0}^{s} b \dot{y}\left(s^{\prime}\right) d s^{\prime}\right),
$$

where $C$ is a constant.

More generally we have.

Proposition 3.17. Consider $(D, g)$ where $D=\operatorname{ker} \omega, \omega=d z-\frac{1}{2} y^{2} d x$ and $g$ is an isoperimetric metric in the normal form $a(x, y) d x^{2}+c(x, y) d y^{2}$. Assume that $a$ and $c$ are not depending on $x$. Then the Hamiltonian vector field $\vec{H}_{n}$ and the associated variational equation are completely integrable.

Proof. Indeed, $H_{n}$ is of the form $\frac{1}{2}\left[\frac{p_{y}^{2}}{c}+\frac{\left(p_{x}+p_{z} \frac{y^{2}}{2}\right)^{2}}{a}\right]$, where $a$ and $c$ are not depending on $x$. Hence we can apply the proposition 3.16 .

\section{The Martinet Flat Case}

\subsection{Preliminaries}

Let $D$ be the distribution ker $\omega$, where $\omega=d z-\frac{1}{2} y^{2} d x$ and $g$ be the flat metric $d x^{2}+d y^{2}, q=(x, y, z)$ being the coordinates in $\mathbb{R}^{3}$. We set $F_{1}=\frac{\partial}{\partial x}+\frac{y^{2}}{2} \frac{\partial}{\partial z}, F_{2}=\frac{\partial}{\partial y}, F_{1}, F_{2}$ being orthonormal vector fields and let $F_{3}=\frac{\partial}{\partial z}$. We set for $i=1,2,3, P_{i}=\left\langle p, F_{i}(q)\right\rangle, p=\left(p_{x}, p_{y}, p_{z}\right)$ being the adjoint vector. The associated control system is

$$
\frac{d q}{d t}(t)=\sum_{i=1}^{2} u_{i}(t) F_{i}(q(t))
$$

and the length and the energy of an admissible curve $\gamma:[0, T] \longmapsto \mathbb{R}^{3}$ are respectively

$$
L(\gamma)=\int_{0}^{T}\left(\sum_{i=1}^{2} u_{i}^{2}(t)\right)^{\frac{1}{2}} d t, \quad E(\gamma)=\int_{0}^{T} \sum_{i=1}^{2} u_{i}^{2}(t) d t
$$

The Hamilton function is

$$
H_{\lambda}=\sum_{i=1}^{2}\left(u_{i} P_{i}-\lambda u_{i}^{2}\right)
$$

where $\lambda=0$ or $\frac{1}{2}$. Normal bi-extremals are solutions corresponding to the Hamilton function $H_{n}=\frac{1}{2}\left(P_{1}^{2}+P_{2}^{2}\right)$. The projections of the abnormal biextremals in the $q$-space are contained in the Martinet plane $y=0$ and are the geodesic lines $y=0, z=z_{0}$.

LEMMA 4.1. The Hamilton equations corresponding to

$$
H_{n}=\frac{1}{2}\left(p_{x}+\frac{y^{2}}{2} p_{z}\right)^{2}+\frac{1}{2} p_{y}^{2}
$$


are

$$
\begin{aligned}
\dot{x} & =p_{x}+\frac{y^{2}}{2} p_{z} & \dot{p}_{x} & =0 \\
\dot{y} & =p_{y} & \dot{p}_{y} & =-\left(p_{x}+\frac{y^{2}}{2} p_{z}\right) p_{z} y \\
\dot{z} & =\left(p_{x}+\frac{y^{2}}{2} p_{z}\right) \frac{y^{2}}{2} & \dot{p}_{z} & =0 .
\end{aligned}
$$

The coordinates $x, z$ are cyclic and $p_{x}, p_{z}$ are first integrals. The equations are integrable by quadratures. A geodesic line $x=t+x_{0}, y=0, z=z_{0}$ is projection of a solution of (4.2) corresponding to $p_{y}=0, p_{x}=1$ and $p_{z}$ arbitrary constant. In particular an abnormal geodesic is not strictly abnormal. Moreover its projection in the $(x, y)$ plane is a geodesic for the induced Riemannian metric $\widetilde{g}=d x^{2}+d y^{2}$.

LEMMA 4.2. In the coordinates $(q, P)$ the normal bi-extremals are solutions of the following equations

$$
\begin{array}{lll}
\dot{x}=P_{1} & \dot{P}_{1}=y P_{2} P_{3} \\
\dot{y}=P_{2} & \dot{P}_{2}=-y P_{1} P_{3} \\
\dot{z}=\frac{y^{2}}{2} P_{1} & \dot{P}_{3}=0,
\end{array}
$$

where $P_{1}=p_{x}+\frac{y^{2}}{2} p_{z}, P_{2}=p_{y}$ and $P_{3}=p_{z}$.

Proof. Since $H_{n}=\frac{1}{2}\left(P_{1}^{2}+P_{2}^{2}\right)$, we obtain

$$
\begin{aligned}
& \dot{P}_{1}=\left\{P_{1}, P_{2}\right\} P_{2} \\
& \dot{P}_{2}=-\left\{P_{1}, P_{2}\right\} P_{1} \\
& \dot{P}_{3}=\left\{P_{3}, P_{1}\right\} P_{1}+\left\{P_{3}, P_{2}\right\} P_{2} .
\end{aligned}
$$

The Poisson brackets are

$$
\left\{P_{1}, P_{2}\right\}=y p_{z}, \quad\left\{P_{1}, P_{3}\right\}=\left\{P_{2}, P_{3}\right\}=0 .
$$

Hence

$$
\dot{P}_{1}=y P_{2} P_{3}, \quad \dot{P}_{2}=-y P_{1} P_{3}, \quad \dot{P}_{3}=0 .
$$

\subsection{NOTATION}

Let us assume that normal bi-extremals are parametrized by arc-length $H_{n}=\frac{1}{2}\left(P_{1}^{2}+P_{2}^{2}\right)=\frac{1}{2}$. We set $P_{1}(0)=\sin \varphi, P_{2}(0)=\cos \varphi$ and $\lambda=$ $P_{3}(t)=P_{3}(0)$. Let $S$ be the group generated by the two diffeomorphisms $S_{1}:(x, y, z) \longmapsto(x,-y, z)$ and $S_{2}:(x, y, z) \longmapsto(-x, y,-z)$.

\subsection{SYMMETRIES}

We observe that $(D, g)$ is left invariant by the action of the group $S$. This imply the following symmetries. If we change $\varphi$ into $\pi-\varphi$, the solution of (4.3): $\left(x(t), y(t), z(t), P_{1}(t), P_{2}(t), P_{3}(t)\right)$ is changed into $\left(x,-y, z, P_{1}\right.$, $\left.-P_{2}, P_{3}\right)$ and the corresponding geodesic is deduced using the symmetry $S_{1}$. Similarly, if we change $\varphi$ into $-\varphi$ and $\lambda$ into $-\lambda$, it is changed into $\left(-x, y,-z,-P_{1}, P_{2},-P_{3}\right)$ and the geodesic is deduced using the symmetry Esaim: CoCv, December 1997, Vol. 2, PP. $377-448$ 
$S_{2}$. Hence when computing the normal geodesics we can assume $\left.\varphi \in\right]-\pi / 2$, $+\pi / 2]$ and $\lambda \geq 0$.

\subsection{Quasi-Homogeneity}

The equations (4.3) are invariant for the following quasi-homogeneous transformations

$$
\begin{aligned}
X & =\varepsilon^{-1} x, \quad Y=\varepsilon^{-1} y, \quad Z=\varepsilon^{-3} z, \\
Q_{1} & =\varepsilon^{-1} P_{1}, \quad Q_{2}=\varepsilon^{-1} P_{2}, \quad Q_{3}=\varepsilon P_{3},
\end{aligned}
$$

and the length is changed as follows

$$
L(X, Y, Z)=\varepsilon^{-1} L(x, y, z) .
$$

Hence if we use the following gradation: the variables $x, y, P_{1}, P_{2}$ have weight 1 , the weight of $z$ is 3 and the weight of $P_{3}$ is -1 . The associated weights for vector fields are -1 for $\frac{\partial}{\partial x}, \frac{\partial}{\partial y}, \frac{\partial}{\partial P_{1}}, \frac{\partial}{\partial P_{2}},-3$ for $\frac{\partial}{\partial z}$ and 1 for $\frac{\partial}{\partial P_{3}}$. Using this gradation, the vector field corresponding to (4.3) has weight 0 and is linear.

Equivalently we can use the gradation: the variables $x, y$ have weight 1 , the variable $z$ a weight 3 , the weight of $P_{1}, P_{2}$ is 0 and weight of $P_{3}$ is -2 , the vector field (4.3) having then the weight -1 and is constant.

\subsection{Parametrization of geodesics}

We assume that the geodesics are parametrized by arc-length and are starting from 0 .

First, observe that equations (4.3) admit the following particular solutions. When $\varphi=\frac{\pi}{2}, x(t)=t, y(t)=z(t)=0$ and it corresponds to the abnormal geodesic. If $\lambda=0, x(t)=t \sin \varphi, y(t)=t \cos \varphi, z(t)=$ $\frac{1}{6} t^{3} \sin \varphi \cos ^{2} \varphi$ and the projection on the plane $(x, y)$ are the lines, which are solutions of the induced Riemannian geometry.

The general solution is computed using elliptic integrals of the first and second kind. We proceed as follows. On the level set $H_{n}=\frac{1}{2}$, we have the equation

$$
\dot{y}^{2}+P_{1}^{2}(y)=1
$$

where $P_{1}=p_{x}+\frac{1}{2} y^{2} p_{z}$ and $p_{x}=\sin \varphi, p_{z}=\lambda$ are constant. This equation describes the evolution of particle of mass 1 and energy 1 , in a potential field where the potential $V$ is $P_{1}^{2}$. The solutions can be computed by considering only the graph of $V$. Equation (4.4) is equivalent to

$$
\dot{y}^{2}=\left(1-P_{1}\right)\left(1+P_{1}\right)=\left(1-p_{x}-\frac{y^{2}}{2} p_{z}\right)\left(1+p_{x}+\frac{y^{2}}{2} p_{z}\right) .
$$

To integrate (4.5), we proceed as follows. Let $0<k, k^{\prime}<1, k^{2}+k^{\prime 2}=1$ defined by

$$
k^{2}=\sin ^{2}\left(\frac{\pi}{4}-\frac{\varphi}{2}\right)=\frac{1-\sin \varphi}{2}, k^{\prime 2}=\cos ^{2}\left(\frac{\pi}{4}-\frac{\varphi}{2}\right)=\frac{1+\sin \varphi}{2},
$$


where $\varphi \in]-\pi / 2,+\pi / 2[$. Hence $y$ satisfies

$$
\dot{y}^{2}=\left(2 k^{2}-\frac{y^{2}}{2} \lambda\right)\left(2 k^{\prime 2}+\frac{y^{2}}{2} \lambda\right) \text {. }
$$

Assume $\lambda>0$ and set $\eta=\frac{y \sqrt{\lambda}}{2 k}$. We get the equation

$$
\frac{\dot{\eta}^{2}}{\lambda}=\left(1-\eta^{2}\right)\left(k^{\prime 2}+k^{2} \eta^{2}\right)
$$

which has to be integrated with the initial condition $\eta(0)=y(0)=0$ and $\dot{\eta}(0)>0$, since $\dot{y}(0)=P_{2}(0)>0$. The solution is periodic and can be written using the Jacobi elliptic functions, see [25], as

$$
\eta(t)=-\operatorname{cn}(K(k)+t \sqrt{\lambda}, k),
$$

where the period is $4 K, K$ being the complete elliptic integral of the first kind:

$$
K(k)=\int_{0}^{1} \frac{d \eta}{\sqrt{\left(1-\eta^{2}\right)\left(k^{\prime 2}+k^{2} \eta^{2}\right)}}=\int_{0}^{\frac{\pi}{2}}\left(1-k^{2} \sin ^{2} \theta\right)^{-\frac{1}{2}} d \theta
$$

Hence $y(t)=-\frac{2 k}{\sqrt{\lambda}} \operatorname{cn}(K(k)+t \sqrt{\lambda}, k)$ and represents a periodic motion whose period is $4 K$ and with the amplitude $2 k / \sqrt{\lambda}$. It is interesting to quote that they are two limit behaviors. When $\varphi \rightarrow \frac{\pi^{-}}{2}, k \rightarrow 0^{+}$and $K(k) \rightarrow \frac{\pi}{2}$. More precisely $\operatorname{cn}(u, k) \rightarrow \cos u$ when $k \rightarrow 0$. The situation is quite different when $\varphi \rightarrow-\frac{\pi^{+}}{2}$, then $k \rightarrow 1^{-}$and $K\left(k^{\prime}\right)=-\log \left(k^{\prime}\right)+0(1)$. Hence $K(k) \rightarrow+\infty$, when $k \rightarrow 1$ and admits a logarithmic singularity.

A mechanical analogy is the following. By setting $P_{1}(t)=\cos \theta(t), P_{2}(t)=$ $\sin \theta(t)$ and using (4.3), we get that $\theta(t)$ is solution of

$$
\dot{\theta}=-\lambda y, \quad \dot{y}=\sin \theta,
$$

which is the equation of a pendulum.

The singular points $\theta=n \pi$ correspond to $\varphi=\frac{\pi}{2}+n \pi$. They are projections of geodesics lines corresponding to abnormal bi-extremals. To geodesics starting from 0 correspond the oscillating motions of the pendulum. In this representation, oscillating solutions tending to the separatrix cycle joining the saddle points and whose period tends to the infinity, encoded the behaviors of geodesics when $k^{\prime} \rightarrow 0$. Those near 0 , which behave like a linearized pendulum correspond to behaviors when $k \rightarrow 0$.

Now, we compute the two other components of a geodesic. We have

$$
\dot{x}=p_{x}+\frac{y^{2}}{2} p_{z}=\sin \varphi+\frac{\lambda y^{2}}{2}=\sin \varphi+2 k^{2} \operatorname{cn}^{2}(K+t \sqrt{\lambda}, k) .
$$

Hence

$$
x(t)=t \sin \varphi+2 k^{2} \int_{0}^{t} \mathrm{cn}^{2}(K+s \sqrt{\lambda}, k) d s .
$$

We set $u=s \sqrt{\lambda}+K$. Recall the formula [25], p. 62:

$$
\int \mathrm{dn}^{2} u d u=k^{\prime 2} u+k^{2} \int \mathrm{cn}^{2} u d u
$$


and let us introduce the Jacobi epsilon function $E(u, k)$ defined by:

$$
E(u, k)=\int_{0}^{u} \mathrm{dn}^{2} v d v .
$$

Therefore, we obtain

$$
\begin{aligned}
k^{2} \int_{K}^{K+t \sqrt{\lambda}} \operatorname{cn}^{2} u d u & =\int_{K}^{K+t \sqrt{\lambda}} \mathrm{dn}^{2} u d u-k^{\prime 2} t \sqrt{\lambda} \\
& =E(K+t \sqrt{\lambda})-E(K)-k^{\prime 2} t \sqrt{\lambda}
\end{aligned}
$$

Hence

$$
x(t)=-t+\frac{2}{\sqrt{\lambda}}(E(K+t \sqrt{\lambda})-E(K)) .
$$

Now we compute the component $z$. We have

$$
\dot{z}=\frac{y^{2}}{2} P_{1}=\left(\sin \varphi+\frac{y^{2}}{2} \lambda\right) \frac{y^{2}}{2}=\frac{y^{4}}{4} \lambda+\frac{y^{2}}{2} \sin \varphi \text {. }
$$

Therefore

$$
z(t)=\frac{4 k^{4}}{\lambda} \int_{0}^{t} \mathrm{cn}^{4}(K(k)+s \sqrt{\lambda}, k) d s+\frac{2 k^{2} \sin \varphi}{\lambda} \int_{0}^{t} \mathrm{cn}^{2}(K(k)+s \sqrt{\lambda}, k) d s .
$$

The first integral is computed with the following formula, see [25] p. 87:

$$
\int \operatorname{cn}^{4} u d u=\frac{1}{3 k^{4}}\left[\left(2-3 k^{2}\right) k^{\prime 2} u+2\left(2 k^{2}-1\right) E(u)+k^{2} \operatorname{sn} u \operatorname{cn} u \operatorname{dn} u\right] .
$$

Hence with $u=K+s \sqrt{\lambda}$, we get

$$
\begin{gathered}
\int_{0}^{t} \operatorname{cn}^{4}(K+s \sqrt{\lambda}) d s=\frac{1}{\sqrt{\lambda}} \int_{K}^{K+t \sqrt{\lambda}} \operatorname{cn}^{4} u d u \\
=\frac{1}{3 k^{4} \sqrt{\lambda}}\left[\left(2-3 k^{2}\right) k^{\prime 2} t \sqrt{\lambda}+2\left(2 k^{2}-1\right)(E(K+t \sqrt{\lambda})-E(K))\right. \\
\left.+k^{2} \operatorname{sn}(K+t \sqrt{\lambda}) \operatorname{cn}(K+t \sqrt{\lambda}) \operatorname{dn}(K+t \sqrt{\lambda})\right]
\end{gathered}
$$

After simplification we obtain

$$
\begin{aligned}
z(t) & =\frac{2}{3 \lambda^{3 / 2}}\left[\left(2 k^{2}-1\right)(E(K+t \sqrt{\lambda})-E(K))+k^{\prime 2} t \sqrt{\lambda}\right. \\
& \left.+2 k^{2} \operatorname{sn}(K+t \sqrt{\lambda}) \operatorname{cn}(K+t \sqrt{\lambda}) \operatorname{dn}(K+t \sqrt{\lambda})\right] .
\end{aligned}
$$

We summarize all the computations into the following proposition.

Proposition 4.3. Arc-length parametrized geodesics starting from 0 are given by

$$
\begin{gathered}
x(t)=-t+\frac{2}{\sqrt{\lambda}}(E(u)-E(K)), y(t)=-\frac{2 k}{\sqrt{\lambda}} \operatorname{cn} u, \\
z(t)=\frac{2}{3 \lambda^{3 / 2}}\left[\left(2 k^{2}-1\right)(E(u)-E(K))+k^{\prime 2} t \sqrt{\lambda}+2 k^{2} \operatorname{sn} u \operatorname{cn} u \mathrm{dn} u\right],
\end{gathered}
$$

where $u=K+t \sqrt{\lambda}, \lambda>0, \varphi \in]-\pi / 2,+\pi / 2[, \operatorname{sn}(u), \operatorname{cn}(u), \operatorname{dn}(u)$ and $E(u)$ being the Jacobi elliptic functions, $K$ and $E(K)$ being the complete elliptic integrals of the first and second kind, or the particular solutions

$$
x(t)=t \sin \varphi, y(t)=t \cos \varphi, z(t)=\frac{t^{3}}{6} \sin \varphi \cos ^{2} \varphi,
$$


where $\varphi \in]-\pi / 2,+\pi / 2]$ and the curves deduced from the previous ones using the symmetries $S_{1}:(x, y, z) \longmapsto(x,-y, z), S_{2}:(x, y, z) \longmapsto(-x, y,-z)$.

REMARK 4.4. The general solution is parametrized using elliptic integrals of the first and second kind. It is an intrinsic measure of the transcendence of the problem. Observe also that $x(t)+t, y(t)$ and $z(t)$ are analytic with respect to the variable $v=t \sqrt{\lambda}$ which can be taken as a new time parameter. REMARK 4.5. We can write $x$ and $z$ as follows

$$
\begin{aligned}
& x(t)=\frac{1}{\sqrt{\lambda}}[L(t \sqrt{\lambda})+P(t \sqrt{\lambda})], \\
& z(t)=\frac{2}{3 \lambda^{\frac{3}{2}}}\left[L^{\prime}(t \sqrt{\lambda})+P^{\prime}(t \sqrt{\lambda})\right]
\end{aligned}
$$

where $L, L^{\prime}$ are linear functions and $P, P^{\prime}$ are $2 \mathrm{~K}$ periodic functions. They are given by

$$
\begin{gathered}
L(v)=\left(\frac{E}{K}-1\right) v, P(v)=2\left(Z(v)-k^{2} \operatorname{sn} v \frac{\operatorname{cn} v}{\operatorname{dn} v}\right), \\
L^{\prime}(v)=\left(\left(2 k^{2}-1\right) \frac{E}{K}+k^{\prime 2}\right) v \\
P^{\prime}(v)=\left(2 k^{2}-1\right)\left(Z(v)-k^{2} \operatorname{sn} v \frac{\operatorname{cn} v}{\operatorname{dn} v}\right)-2 k^{2} k^{\prime 2} \operatorname{sn} v \frac{\operatorname{cn} v}{\operatorname{dn}^{3} v}
\end{gathered}
$$

where $Z$ is the Jacobi Zeta function defined by

$$
Z(v)=E(v)-\frac{E v}{K}
$$

REMARK 4.6. The projections of the normal geodesics on the $(x, y)$ plane are the inflexional elastica described in [30], p. 402.

\subsection{Parametrization of the conjugate locus at 0}

Let $\gamma(t)=(x(t), y(t), z(t))$ be a geodesic computed in the previous section. It is defined on the whole line and we assume $t \in[0,+\infty$ [. When computing the conjugate locus, we can suppose that the projection of $\gamma$ on the plane $(x, y)$ is not a line, because in the strictly normal case such a geodesic is a minimizer and hence without conjugate point. In the abnormal case the whole geodesic is formed with conjugate points. Moreover using the symmetry group, we can assume $\lambda>0$ and $\varphi \in]-\frac{\pi}{2},+\frac{\pi}{2}[$, other conjugate points being deduced using a symmetry $S_{1}:(x, y, z) \longmapsto(x,-y, z)$ or $S_{2}:(x, y, z) \longmapsto(-x, y,-z)$. To compute the conjugate locus $C(0)$, we use the reduction procedure described in section 3.1.1. The first conjugate time $t_{1 c}$, along $\gamma$ is the first $t>0$ such that

$$
c(t, \varphi, \lambda)=0
$$

where

$$
c=\frac{\partial x}{\partial \lambda} \frac{\partial y}{\partial \varphi}-\frac{\partial y}{\partial \lambda} \frac{\partial x}{\partial \varphi}
$$

and

$$
x(t)=-t+\frac{2}{\sqrt{\lambda}}(E(u)-E(K)), y(t)=-\frac{2 k}{\sqrt{\lambda}} \operatorname{cn} u,
$$


with $u=K(k)+t \sqrt{\lambda}, k=\sin \left(\frac{\pi}{4}-\frac{\varphi}{2}\right)$ and $k^{\prime}=\cos \left(\frac{\pi}{4}-\frac{\varphi}{2}\right), \lambda>0$, $\varphi \in]-\frac{\pi}{2},+\frac{\pi}{2}[$. Let us evaluate $c$. Using

$$
\frac{\partial u}{\partial \lambda}=\frac{t}{2 \sqrt{\lambda}}, \frac{\partial E}{\partial u}(u)=\operatorname{dn}^{2} u
$$

we obtain

$$
\frac{\partial x}{\partial \lambda}=\frac{1}{\lambda^{3 / 2}}\left[E(K)-E(u)+t \sqrt{\lambda} \mathrm{dn}^{2} u\right] .
$$

Similarly using

we get

$$
\frac{\partial \operatorname{cn} u}{\partial u}=-\operatorname{sn} u \operatorname{dn} u
$$

$$
\frac{\partial y}{\partial \lambda}=\frac{k}{\lambda^{3 / 2}}[\operatorname{cn} u+t \sqrt{\lambda} \operatorname{sn} u \operatorname{dn} u] .
$$

The partial derivatives with respect to $\varphi$ are more complex. To compute $\partial x / \partial \varphi$ we need the relations

$$
\frac{d k}{d \varphi}=-\frac{k^{\prime}}{2} \quad \frac{d k^{\prime 2}}{d \varphi}=k k^{\prime}, \cos \varphi=2 k k^{\prime},
$$

the following formulas found in [25] pp. 83 and 84 :

$$
\begin{aligned}
\frac{d K}{d k} & =\frac{1}{k k^{\prime 2}}\left(E(K)-k^{\prime 2} K\right) \\
\frac{d E(K)}{d k} & =\frac{1}{k}(E(K)-K) \\
\frac{\partial E(u)}{\partial k} & =\frac{k}{k^{\prime 2}} \operatorname{sn} u \operatorname{cn} u \operatorname{dn} u-k u \operatorname{sn}^{2} u-\frac{k}{k^{\prime 2}} E(u) \operatorname{cn}^{2} u,
\end{aligned}
$$

hence

$$
\frac{\partial u}{\partial \varphi}=\frac{d K}{d k} \frac{d k}{d \varphi}=-\frac{1}{2 k k^{\prime}}\left(E(K)-k^{\prime 2} K\right),
$$

and the identities below

$$
\begin{aligned}
\operatorname{dn}^{2} u+k^{2} \operatorname{sn}^{2} u & =1 \\
\operatorname{dn}^{2} u-k^{2} \operatorname{cn}^{2} u & =k^{\prime 2} \\
\operatorname{cn}^{2} u+\operatorname{sn}^{2} u & =1 .
\end{aligned}
$$

Endly we get

$$
\frac{\partial x}{\partial \varphi}=\frac{k}{k^{\prime} \sqrt{\lambda}}\left[\operatorname{cn}^{2} u\left(E(u)-E(K)-k^{\prime 2} t \sqrt{\lambda}\right)+k^{\prime 2} t \sqrt{\lambda}-\operatorname{sn} u \operatorname{cn} u \operatorname{dn} u\right] .
$$

Similarly using the following formula from [25] p. 82 :

$$
\frac{\partial \operatorname{cn} u}{\partial k}=-\frac{1}{k} u \operatorname{sn} u \operatorname{dn} u-\frac{k}{k^{\prime 2}} \operatorname{sn}^{2} u \operatorname{cn} u+\frac{1}{k k^{\prime 2}} E(u) \operatorname{sn} u \operatorname{dn} u,
$$

we obtain

$$
\frac{\partial y}{\partial \varphi}=-\frac{1}{k^{\prime} \sqrt{\lambda}}\left[\operatorname{sn} u \operatorname{dn} u\left(E(K)+k^{\prime 2} t \sqrt{\lambda}-E(u)\right)+\operatorname{cn} u\left(k^{2} \operatorname{sn}^{2} u-k^{\prime 2}\right)\right] .
$$


Now, we use the following formulas [25] pp . 28 and 67 :

$$
\begin{aligned}
\operatorname{sn}(u+K) & =\operatorname{cd} u=\frac{\operatorname{cn} u}{\operatorname{dn} u} \\
\operatorname{cn}(u+K) & =-k^{\prime} \operatorname{sd} u=-k^{\prime} \frac{\operatorname{sn} u}{\operatorname{dn} u} \\
\operatorname{dn}(u+K) & =k^{\prime} \operatorname{nd} u=\frac{k^{\prime}}{\operatorname{dn} u} \\
E(u+K) & =E(u)+E(K)-\frac{k^{2} \operatorname{sn} u \operatorname{cn} u}{\operatorname{dn} u},
\end{aligned}
$$

and setting $v=t \sqrt{\lambda}$, we obtain

$$
\begin{aligned}
\frac{\partial x}{\partial \lambda} & =\frac{1}{\lambda^{3 / 2}}\left[\frac{k^{2} \operatorname{sn} v \operatorname{cn} v}{\operatorname{dn} v}-E(v)+\frac{k^{\prime 2} v}{\operatorname{dn}^{2} v}\right] \\
\frac{\partial y}{\partial \lambda} & =\frac{k k^{\prime}}{\lambda^{3 / 2} \operatorname{dn} v}\left[\frac{v \operatorname{cn} v}{\operatorname{dn} v}-\operatorname{sn} v\right] \\
\frac{\partial x}{\partial \varphi} & =\frac{k k^{\prime}}{\sqrt{\lambda}}\left[\frac{\operatorname{sn}^{2} v}{\operatorname{dn}^{2} v}\left(E(v)-\frac{k^{2} \operatorname{sn} v \operatorname{cn} v}{\operatorname{dn} v}-k^{\prime 2} v\right)+v+\frac{\operatorname{cn} v \operatorname{sn} v}{\operatorname{dn}^{3} v}\right] \\
\frac{\partial y}{\partial \varphi} & =-\frac{1}{\sqrt{\lambda} \operatorname{dn} v}\left[\frac{\operatorname{cn} v}{\operatorname{dn} v}\left(k^{\prime 2} v-E(v)\right)+k^{\prime 2} \operatorname{sn} v\right] .
\end{aligned}
$$

Hence, after tedious simplifications we can write

$$
c(t, \lambda, \varphi)=\frac{\partial x}{\partial \lambda} \frac{\partial y}{\partial \varphi}-\frac{\partial x}{\partial \varphi} \frac{\partial y}{\partial \lambda}=-\frac{1}{\lambda^{2} \operatorname{dn} v}\left[v^{2} c_{1}(v)+v c_{2}(v)+c_{3}(v)\right],
$$

where

$$
\begin{aligned}
& c_{1}(v)=\frac{k^{\prime 2} \operatorname{cn} v}{\operatorname{dn} v} \\
& c_{2}(v)=k^{\prime 2} \operatorname{sn} v-2 k^{\prime 2} E(v) \frac{\operatorname{cn} v}{\operatorname{dn} v} \\
& c_{3}(v)=E^{2}(v) \frac{\operatorname{cn} v}{\operatorname{dn} v}-E(v) \operatorname{sn} v .
\end{aligned}
$$

THEOREM 4.7. Let $\gamma$ be a geodesic starting at $t=0$ from 0 and defined on $[0,+\infty$. If the projection of $\gamma$ in the plane $(x, y)$ is not a line, then there exists a first conjugate point along $\gamma$ corresponding to a conjugate time $t_{1 c}$ which satisfies the inequality $2 K<t_{1 c} \sqrt{\lambda}<3 K$. Numerical simulations show that the ratio $\frac{t_{1 c} \sqrt{\lambda}}{3 K}$ is roughly a constant equals to $0.9 \%$.

Proof. Let

where

$$
c=-\frac{F(v)}{\lambda^{2} \mathrm{dn}^{2} v},
$$

$$
F(v)=\operatorname{cn} v G_{1}(v)-\operatorname{sn} v \operatorname{dn} v\left(E(v)-k^{\prime 2} v\right)
$$

and

$$
G_{1}(v)=E^{2}(v)-2 k^{\prime 2} v E(v)+k^{\prime 2} v^{2} .
$$

First assume $0<v<K$, we shall prove that $F$ has no zero. We compute $F^{\prime}$ and after simplifications we get

$F^{\prime}(v)=-\operatorname{sn} v \operatorname{dn} v G_{1}(v)-k^{2} \operatorname{sn} v \operatorname{cn}^{2} v \operatorname{dn} v+k^{\prime 2} \operatorname{cn} v(v-E(v))+k^{2} \operatorname{cn} v E(v)$. Esaim: Cocv, December 1997, Vol. 2, PP. 377-448 
Using the following formula from [25], p. 62 :

$$
E(v)=k^{\prime 2} v+\frac{\operatorname{sn} v \operatorname{dn} v}{\operatorname{cn} v}-k^{\prime 2} \int_{0}^{v} \frac{d u}{\operatorname{cn}^{2} u}
$$

which is valid for $0<v<K, F^{\prime}$ can be written

$$
F^{\prime}(v)=F_{1}(v)+F_{2}(v)
$$

where

$$
\begin{aligned}
& F_{1}(v)=-\operatorname{sn} v \operatorname{dn} v\left(G_{1}(v)-k^{2} \operatorname{sn}^{2} v\right), \\
& F_{2}(v)=k^{\prime 2} \operatorname{cn} v F_{3}(v) \\
& F_{3}(v)=v-E(v)+k^{2} v-k^{2} \int_{0}^{v} \frac{d u}{\operatorname{cn}^{2} u} .
\end{aligned}
$$

We claim that $F_{2}(v)<0$ for $0<v<K$.

We have $F_{3}(0)=0$ and moreover

$$
F_{3}^{\prime}(v)=k^{2} \operatorname{sn}^{2} v-\frac{k^{2} \operatorname{sn}^{2} v}{\operatorname{cn}^{2} v}
$$

which is $<0$ since $\operatorname{cn} v<1$. Now using $\operatorname{cn} v>0$ we get $F_{2}(v)<0$.

We claim that $F_{1}(v)<0$ for $0<v<K$. Since $\operatorname{sn} v \operatorname{dn} v>0$, if we set $G_{2}(v)=k^{2} \operatorname{sn}^{2} v$, we must prove that $G_{1}(v)-G_{2}(v)>0$.

First we observe that

$$
G_{1}(0)=G_{2}(0)=0 .
$$

Hence if $G_{1}^{\prime}(v)>G_{2}^{\prime}(v)$, we have $G_{1}(v)>G_{2}(v)$. Computing we get

$$
\begin{aligned}
& G_{1}^{\prime}(v)=2 k^{2} E(v) \operatorname{cn}^{2} v+2 k^{2} k^{\prime 2} v \operatorname{sn}^{2} v, \\
& G_{2}^{\prime}(v)=2 k^{2} \operatorname{cn} v \operatorname{sn} v \operatorname{dn} v .
\end{aligned}
$$

We must prove $H_{1}(v)>H_{2}(v)$, where

$$
\begin{aligned}
& H_{1}(v)=E(v) \operatorname{cn}^{2} v+k^{\prime 2} v \operatorname{sn}^{2} v \\
& H_{2}(v)=\operatorname{sn} v \operatorname{cn} v \operatorname{dn} v .
\end{aligned}
$$

For $0<v<K, \operatorname{dn} v>\operatorname{cn} v$ see [25] p. 49. Integrating we get $E(v)>\operatorname{sn} v$. Moreover $\operatorname{sn} v>0$, hence it is sufficient to prove $I_{1}(v)>I_{2}(v)$, where

$$
I_{1}(v)=\operatorname{cn}^{2} v+k^{\prime 2} v \operatorname{sn} v, I_{2}(v)=\operatorname{cn} v \operatorname{dn} v .
$$

We observe that $I_{1}(0)=I_{2}(0)=1$. Computing and simplifying we have

$$
I_{1}^{\prime}(v)-I_{2}^{\prime}(v)=2 \operatorname{sn} v \operatorname{dn}^{2} v-2 \operatorname{sn} v \operatorname{cn} v \operatorname{dn} v+k^{\prime 2} v \operatorname{cn} v \operatorname{dn} v .
$$

Using again $\mathrm{dn} v>\operatorname{cn} v$, we obtain $I_{1}^{\prime}(v)>I_{2}^{\prime}(v)$. Therefore $I_{1}(v)>I_{2}(v)$ and the result is proved.

Moreover $F(K)=-\operatorname{sn} K \mathrm{dn} K\left(E(K)-k^{\prime 2} K\right)$ and $E(K)-k^{\prime 2} K>0$ for $0<k<1$, see $[25]$ p. 74 . Then we have $F(K) \neq 0$. Hence we have proved that for $0<v \leq K, F(v)$ is non zero.

Now, let us prove that $F$ is non zero for $K \leq v \leq 2 K$. From the previous computations $G_{1}(v)>0$ for each $v>0$. Indeed $G_{1}^{\prime}(v)>0$ and $G_{1}(0)=0$. Recall that

$$
F(v)=\operatorname{cn} v G_{1}(v)-\operatorname{sn} v \operatorname{dn} v\left(E(v)-k^{\prime 2} v\right)
$$


and for $K \leq v \leq 2 K, \operatorname{sn} v, \operatorname{dn} v, E(v)-k^{\prime 2} v$ are positive, $\operatorname{cn} v$ is negative and the zeros of $\operatorname{sn} v$ and $\operatorname{cn} v$ are distinct. Hence we have

$$
F(v)<0 \text { for } K \leq v \leq 2 K .
$$

We prove that $F$ vanishes for $2 K<v<3 K$. We observe that for $v=K$ and $3 K, F$ reduces to

$$
\begin{aligned}
F(K) & =-\operatorname{sn} K \operatorname{dn} K\left(E(K)-k^{\prime 2} K\right) \\
F(3 K) & =-\operatorname{sn}(3 K) \operatorname{dn}(3 K)\left(E(3 K)-3 k^{\prime 2} K\right) .
\end{aligned}
$$

Using the following formulas from [25], pp. 28 and 64 :

$$
\operatorname{sn} 3 K=-\operatorname{sn} K, \operatorname{dn} 3 K=\operatorname{dn} K, E(3 K)=3 E(K),
$$

we get

$$
F(3 K)=-3 F(K) \neq 0 .
$$

Since $F$ has opposite signs for $K$ and $3 K$, it vanishes for $K<v<3 K$.

We proved that $F$ has no zero for $0<v \leq 2 K$ and $F$ vanishes for $2 K<v<3 K$. The theorem is proved. On figure 24 in the appendix we compute experimentally the quotient $\frac{t_{1 c} \sqrt{\lambda}}{3 K}$ which is roughly a constant $\simeq$ 0,97 .

\subsection{Cut-Locus - Preliminaries}

We shall describe now the cut-locus. The main property to be proved is that geodesics do not intersect before cutting the Martinet plane $y=0$. The system is represented as a pendulum

$$
\begin{aligned}
& \begin{array}{ll}
\dot{x}=\cos \theta & \dot{\theta}=-\lambda y
\end{array} \\
& \begin{array}{ll}
\dot{y}=\sin \theta & \dot{\lambda}=0
\end{array} \\
& \dot{z}=\frac{y^{2}}{2} \cos \theta \text {. }
\end{aligned}
$$

In our study, we can consider geodesic segments which are contained in the half-space $y<0$. Assume $\lambda>0$. The angle $\theta$ increases monotonously along geodesics while $y<0$ and we parametrize geodesics by $\theta$ instead of length.

Let $\theta \longmapsto(x(\theta), y(\theta), z(\theta))$ be a geodesic such that $x\left(\theta_{0}\right)=y\left(\theta_{0}\right)=$ $z\left(\theta_{0}\right)=0$, where $\left.\theta_{0} \in\right]-\pi, 0[$, when $\dot{y}(0)<0$. From (4.23) we get

$$
\begin{aligned}
& x(\theta)=\frac{1}{\sqrt{2} \lambda^{\frac{1}{2}}} \int_{\theta_{0}}^{\theta} \frac{\cos t}{\varphi\left(\theta_{0}, t\right)} d t \\
& y(\theta)=-\sqrt{2} \lambda^{-\frac{1}{2}} \varphi\left(\theta_{0}, \theta\right) \\
& z(\theta)=\frac{1}{\sqrt{2} \lambda^{\frac{3}{2}}} \int_{\theta_{0}}^{\theta} \varphi\left(\theta_{0}, t\right) \cos t d t
\end{aligned}
$$

where $\varphi\left(\theta_{0}, t\right)=\left(\cos t-\cos \theta_{0}\right)^{1 / 2}$ and $y(\theta)<0$ for $\theta_{0}<\theta<-\theta_{0}$.

Let $\mathcal{D}$ be the domain $\left\{\xi=\left(\theta_{0}, \theta\right) ;-\pi<\theta_{0}<0, \theta_{0}<\theta<-\theta_{0}\right\}$. Let $q:(\xi, \lambda) \in \mathcal{D} \times] 0,+\infty\left[\rightarrow \mathbb{R}^{3}\right.$ be the mapping defined by the formulas (4.24). We are going to prove that $q$ is a one-to-one mapping.

We proceed as follows. First we eliminate the parameter $\lambda$ and the coordinate $y$ using the quasi-homogeneity property, see section 4.4. Indeed, suppose that $q\left(\theta^{1}, \theta_{0}^{1}, \lambda^{1}\right)=q\left(\theta^{2}, \theta_{0}^{2}, \lambda^{2}\right)$, then $q\left(\theta^{1}, \theta_{0}^{1}, \varepsilon \lambda^{1}\right)=q\left(\theta^{2}, \theta_{0}^{2}, \varepsilon \lambda^{2}\right)$, $\forall \varepsilon>0$. Multiplying $\lambda$ by $\varepsilon$, we multiply the second coordinate by $\varepsilon^{-\frac{1}{2}}$. It Esaim: CoCv, December 1997, Vol. 2, PP. $377-448$ 
permits us to look for intersections points only in the affine space $y=-1$. If $y(\theta)=-1$, then $\lambda^{\frac{1}{2}}=\sqrt{2} \varphi\left(\theta_{0}, \theta\right)$.

We define the mapping $Q$ as follows: $Q(\xi)=(X(\xi), Z(\xi))$ where

$$
\begin{aligned}
& X(\xi)=\frac{1}{2 \varphi(\xi)} \int_{\theta_{0}}^{\theta} \frac{\cos t}{\varphi\left(\theta_{0}, t\right)} d t \\
& Z(\xi)=\frac{1}{4 \varphi^{3}(\xi)} \int_{\theta_{0}}^{\theta} \varphi\left(\theta_{0}, t\right) \cos t d t .
\end{aligned}
$$

If we introduce

$$
\begin{aligned}
A(\xi) & =\int_{\theta_{0}}^{\theta} \varphi\left(\theta_{0}, t\right) d t \\
B(\xi) & =\int_{\theta_{0}}^{\theta} \frac{d t}{\varphi\left(\theta_{0}, t\right)}, \\
C(\xi) & =\int_{\theta_{0}}^{\theta} \varphi^{3}\left(\theta_{0}, t\right) d t
\end{aligned}
$$

we can write

$$
\begin{aligned}
X(\xi) & =\frac{1}{2 \varphi(\xi)}\left[A(\xi)+\cos \theta_{0} B(\xi)\right] \\
Z(\xi) & =\frac{1}{4 \varphi^{3}(\xi)}\left[C(\xi)+\cos \theta_{0} A(\xi)\right] .
\end{aligned}
$$

We derive from the estimate of theorem 4.7 concerning conjugate points, $t_{1 c} \sqrt{\lambda}>2 K$, that $\frac{\partial Q}{\partial \theta} \wedge \frac{\partial Q}{\partial \theta_{0}} \neq 0$ if $y(t)<0$. Hence $Q$ is locally one-to-one. The problem is to prove a global version of this property.

Proposition 4.8. $Q$ is a diffeomorphism of the domain $\mathcal{D}=\left\{\xi=\left(\theta_{0}, \theta\right) ;-\pi<\theta_{0}<0, \theta_{0}<\theta<-\theta_{0}\right\}$ onto the domain $\mathcal{R}=$ $\{(x, z): x<6 z\}$.

Proof. Let $L$ be the line: $x-6 z=0$. A main step in the proof is to show that the boundary of the Image of $Q$ is the line $L$.

Let $m \in \partial \operatorname{Im} Q, \partial \operatorname{Im} Q \subset \overline{\operatorname{Im} Q}$, hence there exists a sequence $\xi_{n} \in \mathcal{D}$ such that $m$ is the limit of the sequence $Q\left(\xi_{n}\right)$. Since $\mathcal{D}$ is relatively compact, by choosing a subsequence, we can assume that $\xi_{n}$ converges in $\mathbb{R}^{2}$ to a point $\xi_{\infty}$. If $\xi_{\infty}$ is interior to $\mathcal{D}$, there exists an open set $U$ in $\mathcal{D}$ such that $\xi_{\infty} \in U$. Since $Q$ is an open mapping, $Q(U)$ is an open set containing $Q\left(\xi_{\infty}\right)$ and by continuity $m=Q\left(\xi_{\infty}\right)=\lim _{n \rightarrow+\infty} Q\left(\xi_{n}\right)$. This contradicts the fact that $m \in \partial \operatorname{Im} Q$. Hence necessarily $\xi_{\infty} \in \partial \mathcal{D}$.

We write $\partial \mathcal{D}=\triangle_{+} \cup \triangle_{-} \cup \triangle_{0}$ where:

$$
\begin{gathered}
\triangle_{+}=\left\{\theta=-\theta_{0} ;-\pi \leq \theta_{0} \leq 0\right\}, \triangle_{-}=\left\{\theta=\theta_{0} ;-\pi \leq \theta_{0} \leq 0\right\}, \\
\triangle_{0}=\left\{\theta_{0}=-\pi ;-\pi \leq \theta \leq \pi\right\} .
\end{gathered}
$$

We need some auxiliary lemmas to analyse the different cases when $\xi \rightarrow$ $\xi_{\infty}$.

LemMa 4.9. If $\xi \in \mathcal{D}$ and $\xi \rightarrow \xi_{\infty}=(\bar{\theta}, \bar{\theta}),-\pi<\bar{\theta}<0$, then $X(\xi) \rightarrow$ $-\cot \bar{\theta}$ and $Z(\xi) \rightarrow-\frac{1}{6} \cot \bar{\theta}$. Hence $Q$ can be extended by continuity on $\mathcal{D} \cup \Delta_{-} \backslash\{(0,0),(-\pi,-\pi)\}$ and $\overline{\operatorname{Im} Q}$ contains the whole line $L$. 
Proof. Take $t \in\left[\theta_{0}, \theta\right]$ and $\left|\theta_{0}-\bar{\theta}\right|,|\theta-\bar{\theta}|$ small enough. We have:

$$
\begin{gathered}
\varphi\left(\theta_{0}, t\right) \sim \sqrt{-\sin \theta_{0}} \sqrt{t-\theta_{0}} \\
\int_{\theta_{0}}^{\theta} \varphi\left(\theta_{0}, t\right)^{k} d t \sim \frac{2}{k+2}\left(-\sin \theta_{0}\right)^{\frac{k}{2}}\left(\theta-\theta_{0}\right)^{\frac{k+2}{2}}, k \in \mathbb{Z}, k \geq-1 .
\end{gathered}
$$

Hence when $\xi \rightarrow(\bar{\theta}, \bar{\theta}) \in \Delta_{-} \backslash\{(0,0),(-\pi,-\pi)\}$,

$$
\frac{A}{\varphi} \rightarrow 0, \frac{A}{\varphi^{3}} \rightarrow-\frac{2}{3} \frac{1}{\sin \bar{\theta}}, \frac{B}{\varphi} \rightarrow-\frac{2}{\sin \bar{\theta}}, \frac{C}{\varphi^{3}} \rightarrow 0
$$

and $X(\xi) \rightarrow-\cot \bar{\theta}, Z(\xi) \rightarrow-\frac{1}{6} \cot \bar{\theta}$.

Therefore $\overline{\operatorname{Im} Q}$ contains the line $L: x-6 z=0$ and $Q$ can be extended by continuity on $\mathcal{D} \cup \Delta_{-} \backslash\{(0,0),(-\pi,-\pi)\}$.

Lemma 4.10. If $\xi \in \mathcal{D}$ and $\xi \rightarrow(0,0)$ or $\xi \rightarrow(-\pi,-\pi)$, then $X(\xi) \rightarrow \infty$.

Proof. We can write

$$
\varphi\left(\theta_{0}, \theta\right)=\sqrt{\frac{\theta_{0}^{2}-\theta^{2}}{2}} F\left(\theta_{0}, \theta\right),
$$

where $F\left(\theta_{0}, \theta\right) \rightarrow 1$ uniformly on $\mathcal{D}$ when $\xi \rightarrow 0$.

Hence

$$
B(\xi) \sim \int_{\theta_{0}}^{\theta} \frac{\sqrt{2}}{\sqrt{\theta_{0}^{2}-t^{2}}} d t, \text { when } \xi \rightarrow 0 .
$$

To compute the integral we set:

$$
\frac{t}{\left|\theta_{0}\right|}=\cos \psi, \quad \cos \beta=\frac{\theta}{\left|\theta_{0}\right|}, 0<\beta<\pi
$$

and we obtain

$$
B(\xi) \sim \sqrt{2}(\pi-\beta) \text {, when } \xi \rightarrow 0 .
$$

Similarly

$$
A(\xi) \sim \int_{\theta_{0}}^{\theta} \sqrt{\frac{\theta_{0}^{2}-t^{2}}{2}} d t=\frac{\theta_{0}^{2}}{\sqrt{2}} \int_{\beta}^{\pi} \sin ^{2} \psi d \psi
$$

Hence

Moreover

$$
A(\xi) \sim \frac{\theta_{0}^{2}}{\sqrt{2}}\left[\frac{\pi-\beta}{2}+\frac{\sin 2 \beta}{4}\right] \text { when } \xi \rightarrow 0 .
$$

Therefore when $\xi \rightarrow 0$,

$$
\varphi(\xi) \sim \frac{\left|\theta_{0}\right|}{\sqrt{2}} \sin \beta
$$

$$
\frac{A}{\varphi} \sim\left|\theta_{0}\right|\left[\frac{\pi-\beta}{2 \sin \beta}+\frac{\cos \beta}{2}\right], \frac{B}{\varphi} \sim \frac{2}{\left|\theta_{0}\right|} \frac{\pi-\beta}{\sin \beta} .
$$

Since $\frac{\pi-\beta}{\sin \beta} \geq 1$ for $0<\beta<\pi, \frac{B}{\varphi} \rightarrow+\infty$ when $\xi \rightarrow 0$ and $X(\xi) \rightarrow+\infty$.

The case $\xi \rightarrow(-\pi,-\pi)$ is similar.

Lemma 4.11. Let $\xi \in \mathcal{D} \rightarrow \xi_{\infty} \in \Delta_{+} \cup \Delta_{0} \backslash\{(0,0),(-\pi,-\pi)\}$, $\xi_{\infty}=\left(\theta_{0}(\infty), \theta(\infty)\right)$. Then:

i) If $\theta_{0}(\infty)=-\pi, X(\xi) \rightarrow-\infty$.

ii) If $\theta_{0}(\infty) \neq-\pi$ and $A\left(\xi_{\infty}\right)+\cos \theta_{0}(\infty) B\left(\xi_{\infty}\right) \neq 0$, then $X(\xi) \rightarrow \infty$.

iii) If $\theta_{0}(\infty) \neq-\pi$ and $A\left(\xi_{\infty}\right)+\cos \theta_{0}(\infty) B\left(\xi_{\infty}\right)=0$, then $Z(\xi) \rightarrow+\infty$. 
Proof. On $\mathcal{D}, \theta_{0}<\theta<-\theta_{0}$, hence if $\xi_{\infty} \in \Delta_{+} \cup \Delta_{0} \backslash\{(0,0),(-\pi,-\pi)\}$, $\theta_{0}(\infty)<\theta(\infty)$ and if $\xi \rightarrow \xi_{\infty}, \theta_{0}(\infty) \neq-\pi$, we have:

$$
\begin{aligned}
A(\xi) & =\int_{\theta_{0}}^{\theta} \varphi\left(\theta_{0}, t\right) d t \rightarrow \int_{\theta_{0}(\infty)}^{\theta(\infty)} \varphi\left(\theta_{0}(\infty), t\right) d t>0, \\
B(\xi) & =\int_{\theta_{0}}^{\theta} \frac{d t}{\varphi\left(\theta_{0}, t\right)} \rightarrow \int_{\theta_{0}(\infty)}^{\theta(\infty)} \frac{d t}{\varphi\left(\theta_{0}(\infty), t\right)}>0 \\
C(\xi) & =\int_{\theta_{0}}^{\theta} \varphi^{3}\left(\theta_{0}, t\right) d t \rightarrow \int_{\theta_{0}(\infty)}^{\theta(\infty)} \varphi^{3}\left(\theta_{0}(\infty), t\right) d t>0
\end{aligned}
$$

and $\varphi(\xi) \rightarrow 0$. Therefore if $\theta_{0}(\infty) \neq-\pi$ and $A\left(\xi_{\infty}\right)+\cos \theta_{0}(\infty) B\left(\xi_{\infty}\right) \neq 0$ we get:

$$
X(\xi) \sim \frac{A\left(\xi_{\infty}\right)+\cos \theta_{0}(\infty) B\left(\xi_{\infty}\right)}{2 \varphi\left(\xi_{\infty}\right)}
$$

and $X(\xi) \rightarrow \infty$ when $\xi \rightarrow \xi_{\infty}$. This proves ii).

Assume $\theta_{0}(\infty) \neq-\pi$ and $A\left(\xi_{\infty}\right)+\cos \theta_{0}(\infty) B\left(\xi_{\infty}\right)=0$, we shall prove iii). First observe that when $\xi=\left(\theta_{0}, \theta\right), \theta>\theta_{0}$, we have

$$
0<A(\xi)<\sqrt{B(\xi) C(\xi)} .
$$

Indeed if we set $\varphi\left(\theta_{0}, t\right)=g(t) h(t)$ where $g(t)=\varphi\left(\theta_{0}, t\right)^{\frac{3}{2}}$ and $h(t)=$ $\varphi\left(\theta_{0}, t\right)^{-\frac{1}{2}}$, we have $g \neq h$ and from Cauchy-Schwartz inequality

$$
0<A=\int_{\theta_{0}}^{\theta} g(t) h(t) d t<\left(\int_{\theta_{0}}^{\theta} g^{2}(t) d t\right)^{\frac{1}{2}}\left(\int_{\theta_{0}}^{\theta} h^{2}(t) d t\right)^{\frac{1}{2}}
$$

and $C=\int_{\theta_{0}}^{\theta} g^{2}(t) d t, B=\int_{\theta_{0}}^{\theta} h^{2}(t) d t$.

Hence

$$
\begin{aligned}
C\left(\xi_{\infty}\right)+\cos \theta_{0}(\infty) A\left(\xi_{\infty}\right) & =A\left(\xi_{\infty}\right)\left[\frac{C\left(\xi_{\infty}\right)}{A\left(\xi_{\infty}\right)}+\cos \theta_{0}(\infty)\right] \\
& >A\left(\xi_{\infty}\right)\left[\sqrt{\frac{C\left(\xi_{\infty}\right)}{B\left(\xi_{\infty}\right)}}+\cos \theta_{0}(\infty)\right] .
\end{aligned}
$$

Moreover

$$
\cos \theta_{0}(\infty)=-\frac{A\left(\xi_{\infty}\right)}{B\left(\xi_{\infty}\right)}>-\frac{\sqrt{B\left(\xi_{\infty}\right) C\left(\xi_{\infty}\right)}}{B\left(\xi_{\infty}\right)}=-\sqrt{\frac{C\left(\xi_{\infty}\right)}{B\left(\xi_{\infty}\right)}}
$$

Therefore

$$
C\left(\xi_{\infty}\right)+\cos \theta_{0}(\infty) A\left(\xi_{\infty}\right)>0
$$

Since $Z(\xi) \sim \frac{1}{4 \varphi^{3}\left(\xi_{\infty}\right)}\left(C\left(\xi_{\infty}\right)+\cos \theta_{0}(\infty) A\left(\xi_{\infty}\right)\right)$, we deduce that $Z(\xi) \rightarrow$ $+\infty$, when $\xi \rightarrow \xi_{\infty}$.

An easy computation shows that when $\xi \rightarrow \xi_{\infty}, \theta_{0}(\infty)=-\pi$, then $X(\xi) \rightarrow-\infty$. This proves $\mathrm{i})$.

Corollary 4.12. If $\xi \rightarrow \xi_{\infty} \in \partial \mathcal{D}, Q(\xi) \rightarrow \infty$ and $X(\xi)$ is bounded, then $Z(\xi) \rightarrow+\infty$. 
The preceding considerations show that $\operatorname{Im} Q$ is an open connected set whose boundary is contained in the line $L$ and $\overline{\operatorname{Im} Q}$ contains the line $L$ : $\{x-6 z=0\}$.

Assume that $\partial \operatorname{Im} Q \neq L$, then from lemma 4.9 , there exists $\left(x_{0}, z_{0}\right) \in L$ such that $\left(x_{0}, z_{0}\right)$ belongs to $\operatorname{Im} Q$. Hence $\operatorname{Im} Q$ contains a segment $\left(x_{0},\left[z_{0}-\right.\right.$ $\left.\varepsilon, z_{0}+\varepsilon\right]$ ) and since $\partial \operatorname{Im} Q$ is contained in $L$, it contains the whole line $C: x=x_{0}$. Therefore we can construct a sequence $\xi_{n} \in \mathcal{D}$ converging to $\xi_{\infty} \in \partial \mathcal{D}$ such that $Q\left(\xi_{n}\right) \in C$ and $Z\left(\xi_{n}\right) \rightarrow-\infty$. By construction $X\left(\xi_{n}\right)=x_{0}$ and hence $X\left(\xi_{n}\right)$ is bounded. This contradicts the assertion of the corollary 4.12. Therefore $\partial \operatorname{Im} Q=L$.

Since $\partial \operatorname{Im} Q=L, \operatorname{Im} Q$ is one of the half-space $x-6 z<0$ or $x-6 z>0$. Using again the corollary, we see that $\operatorname{Im} Q$ is the half-space $x-6 z<0$.

The mapping $Q$ is clearly proper. Indeed take a compact $K$ in $\mathcal{R}$, and $\xi_{n}$ a sequence in $Q^{-1}(K)$ converging to $\xi_{\infty}$ in $\mathbb{R}^{2}$. The previous lemmas show that $\xi_{\infty}$ is not in $\partial \mathcal{D}$. Hence the closure of $Q^{-1}(K)$ in $\mathbb{R}^{2}$ doesn't intersect the boundary of $\mathcal{D}$. Therefore $Q^{-1}(K)$ is a compact subset of $\mathcal{D}$.

The mapping $Q$ is a proper local homeomorphism from $\mathcal{D}$ onto $\operatorname{Im} Q=$ $\{x-6 z<0\}$, hence it is a covering. Since $\operatorname{Im} Q$ is $\operatorname{simply}$ connected it is a diffeomorphism and the proposition is proved.

LEMMA 4.13. Let $\gamma(),. \hat{\gamma}($.$) be two distinct geodesics parametrized by the$ length, $\gamma(0)=\hat{\gamma}(0), \gamma(t)=\hat{\gamma}(\hat{t})$, for some $t \geq \hat{t}>0$. Then, $\left.\gamma\right|_{[0, \tau[}$ is not a length minimizer, for any $\tau>\frac{t+\hat{t}}{2}$.

Proof. The statement is obvious if $t>\hat{t}$. Suppose $t=\hat{t}$ and $\left.\gamma\right|_{[0, \tau[\text { is a length }}$ minimizer for some $\tau>t$.

Then, the broken curve

$$
s \mapsto \begin{cases}\hat{\gamma}(s), & 0 \leq s \leq t \\ \gamma(s), & t \leq s \leq \tau\end{cases}
$$

is also a length minimizer. The curve (4.27) is not however a geodesic and it cannot be the length minimizer.

THEOREM 4.14. The geodesics whose projection in the plane $(x, y)$ is a line are minimizers. A geodesic whose projection is not a line admits a cut point at time $t_{p}=2 \mathrm{~K} / \sqrt{\lambda}$ corresponding to its first intersection with Martinet surface. Hence the intersection of the conjugate locus and the cut locus is empty.

The proof is a consequence of the following geometric considerations.

\subsection{A PREview of the sub-Riemannian SpHere}

A nice flat representation of the sphere (a chart) is indeed provided by mapping $Q$. First, consider the particular geodesics given by $x(t)=$ $P_{1} t, y(t)=P_{2} t$ and $z(t)=\frac{1}{6} P_{1} P_{2}^{2} t^{3}$, where $P_{1}$ and $P_{2} \neq 0$ are constant. They project onto lines in the plane $(x, y)$. Hence they are minimizers. Assume $P_{2}<0$ and consider their intersections with the plane $y=-1$. We get $z=x / 6$ and they form the boundary of the domain $\mathcal{R}$.

Now, consider an arc-length parametrized geodesic whose projection in the plane $(x, y)$ is not a line and associated to $(\varphi, \lambda)$ in the parametrization of proposition 4.3. We can assume $\lambda>0$ and $\varphi \in]-\frac{\pi}{2}, \frac{\pi}{2}[$. It is ESAIM: CoCV, December 1997, Vol. 2, PP. 377-448 
well known that it is a minimizer for small time. Its $y$ coordinate is given by $y(t)=-\frac{2 k}{\sqrt{\lambda}} \operatorname{cn}(\mathrm{K}+t \sqrt{\lambda}, k)$. The first intersection with Martinet surface corresponds to $t \sqrt{\lambda}=2 \mathrm{~K}$. Now using the symmetry $S_{1}:(x, y, z) \mapsto$ $(x,-y, z)$, it intersects also at the same point and at the same time the geodesic corresponding to $\lambda$ and $\pi-\varphi$. Moreover the respective slopes are $\left(P_{1}, P_{2}, 0\right)$ and $\left(P_{1},-P_{2}, 0\right)$. From lemma 4.13 , the geodesic cannot be minimizing beyond this intersection point. Now from our previous study using mapping $Q$, we have a very precise representation of geodesics contained in one half-space $y \neq 0$.

We can assume for instance $y<0$. Cutting by $y=-1$, and using mapping $Q$, we see that the geodesic projections corresponding to $\lambda>0$ filled the domain $\mathcal{R}: 6 z>x$, without intersecting. It is represented on figure 1 . The boundary $6 z=x$ contains the geodesics corresponding to $\lambda=0, \varphi \neq k \frac{\pi}{2}$ which project onto line in the plane $(x, y)$, and domain $\overline{\mathcal{R}}: 6 z<x$ is filled with projections corresponding to $\lambda<0$.

In particular, before intersecting Martinet surface, taking a point $q_{1}$ on the geodesic, there is no other geodesic path joining 0 to $q_{1}$. Hence it is minimizing.

Moreover our study gives us a precise representation of minimizing geodesics which is in fact equivalent to a chart of the sphere.

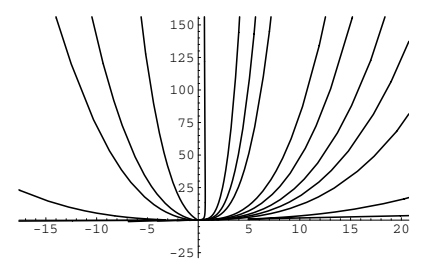

FIGURE 1

\subsection{Sub-Riemannian sphere $\mathrm{S}(0, r)$}

4.9.1. Intersection of $\mathrm{S}(0, r)$ with the cUt Locus. This intersection can be precisely computed using the previous section. If we take geodesics parametrized by arc-length, the time is the length. Hence, let $r>0$, setting $t_{p}=r=2 \mathrm{~K} / \sqrt{\lambda}$ which corresponds to the first intersection with Martinet surface and using the parametrization coming from proposition 4.3 , we get

$$
\begin{aligned}
& x(k)=-r+\frac{2}{\sqrt{\lambda}}(\mathrm{E}(3 \mathrm{~K})-\mathrm{E}(\mathrm{K})) \\
& z(k)=\frac{2}{3 \lambda^{\frac{3}{2}}}\left[\left(2 k^{2}-1\right)(\mathrm{E}(3 \mathrm{~K})-\mathrm{E}(\mathrm{K}))+2 \mathrm{~K} k^{\prime 2}\right] .
\end{aligned}
$$

Using $\mathrm{E}(3 \mathrm{~K})=3 \mathrm{E}(\mathrm{K})$, and the notation $\mathrm{E}$ for $\mathrm{E}(\mathrm{K})$, we get that the intersection of the cut locus with the sphere $S(0, r)$ is a curve denoted $k \mapsto c(k)$, contained in Martinet plane $y=0$, which admits the following parametric 
representation

$$
\begin{aligned}
& x(k)=-r+2 r \frac{\mathrm{E}}{\mathrm{K}} \\
& z(k)=\frac{r^{3}}{6 \mathrm{~K}^{3}}\left[\left(2 k^{2}-1\right) \mathrm{E}+k^{\prime 2} \mathrm{~K}\right]
\end{aligned}
$$

where $k \in] 0,1[$, and the curve deduced from $c$ using the symmetry $(x, z) \mapsto$ $(-x,-z)$.

In order to understand the properties of this curve, the following properties are fundamental.

First, by definition

$$
\mathrm{K}(k)=\int_{0}^{1} \frac{d \eta}{\sqrt{\left(1-\eta^{2}\right)\left(k^{\prime 2}+k^{2} \eta^{2}\right)}}=\int_{0}^{\frac{\pi}{2}}\left(1-k^{2} \sin ^{2} \theta\right)^{-\frac{1}{2}} d \theta
$$

and

$$
\mathrm{E}(k)=\int_{0}^{\mathrm{K}} \mathrm{dn}^{2}(u) d u=\int_{0}^{\frac{\pi}{2}}\left(1-k^{2} \sin ^{2} \theta\right)^{\frac{1}{2}} d \theta .
$$

Both mappings can be expanded in ascending powers of $k^{2}$, see [25] p. 73,

$$
\begin{aligned}
\mathrm{K} & =\frac{1}{2} \pi\left[1+\left(\frac{1}{2}\right)^{2} k^{2}+\left(\frac{13}{24}\right)^{2} k^{4}+\ldots\right] \\
\mathrm{E} & =\frac{1}{2} \pi\left[1-\left(\frac{1}{2}\right)^{2} k^{2}-\frac{1}{3}\left(\frac{13}{24}\right)^{2} k^{4}-\ldots\right]
\end{aligned}
$$

for $0<k<1$.

Hence

$$
\frac{\mathrm{E}}{\mathrm{K}}=1-\frac{k^{2}}{2}+o\left(k^{2}\right)
$$

and

$$
\begin{aligned}
x(k)-r & =-r k^{2}+o\left(k^{2}\right) \\
z(k) & =\frac{2 r^{3}}{3 \pi^{2}} k^{2}+o\left(k^{2}\right) .
\end{aligned}
$$

In particular, when $k \rightarrow 0^{+}$, the cut locus intersected with the sphere is the restriction to $z>0$ of the graph of an analytic function which can be represented by

$$
z=-\frac{2 r^{2}}{3 \pi^{2}}(x-r)+o(x-r)
$$

When $k \rightarrow 1^{-}$, the situation is quite different. This is due to the following fact. We can extend the mapping $k \mapsto \mathrm{K}(k)$ to an analytic function on $\mathbb{C} \backslash[1,+\infty[$, which presents a logarithmic singularity when $k \rightarrow 1$. More precisely, computing we get the following asymptotic expansions, when $k^{\prime} \rightarrow$ 0

$$
\begin{aligned}
& \mathrm{K}=\log \left(\frac{1}{k^{\prime}}\right)+\log 4+o\left(k^{\prime}\right) \\
& \mathrm{E}=1+\frac{k^{\prime 2}}{2} \log \left(\frac{1}{k^{\prime}}\right)+\frac{k^{\prime} 2}{2}\left(\log 4-\frac{1}{2}\right)+o\left(k^{\prime 3}\right) .
\end{aligned}
$$


To get the complete asymptotic expansion, we can use the following properties see [25], section 3.8. First we have

$$
\begin{aligned}
\frac{d \mathrm{E}}{d k} & =\frac{1}{k}(\mathrm{E}-\mathrm{K}) \\
\frac{d \mathrm{~K}}{d k} & =\frac{1}{k k^{\prime 2}}\left(\mathrm{E}-k^{\prime 2} \mathrm{~K}\right)
\end{aligned}
$$

and $\mathrm{K}$ is solution of the following second-order linear Fuchsian differential equation

$$
k\left(1-k^{2}\right) \frac{d^{2} w}{d k^{2}}+\left(1-3 k^{2}\right) \frac{d w}{d k}-k w=0
$$

and $\mathrm{E}$ is solution of the same kind of equation

$$
k\left(1-k^{2}\right) \frac{d^{2} w}{d k^{2}}+\left(1-k^{2}\right) \frac{d w}{d k}+k w=0 .
$$

This can be used to compute the asymptotic expansions when $k \rightarrow 1$ and we get, see [13] p. 134:

$$
\begin{gathered}
K(k)=\log \left(\frac{4}{k^{\prime}}\right)+\left(\frac{1}{2}\right)^{2} k^{\prime 2}\left[\log \left(\frac{4}{k^{\prime}}\right)-1\right]+\left(\frac{1.3}{2.4}\right)^{2} k^{\prime} 4\left[\log \left(\frac{4}{k^{\prime}}\right)-1-\frac{2}{3.4}\right] \\
+\left(\frac{1.3 .5}{2.4 .6}\right)^{2} k^{\prime}\left[\log \left(\frac{4}{k^{\prime}}\right)-1-\frac{2}{3.4}-\frac{2}{5.6}\right]+\ldots, \\
E(k)=1+\left(\frac{1}{2}\right)^{2} k^{\prime}\left[\log \left(\frac{4}{k^{\prime}}\right)-\frac{1}{1.2}\right]+\left(\frac{1}{2}\right)^{2} \frac{3}{4} k^{\prime}{ }^{4}\left[\log \left(\frac{4}{k^{\prime}}\right)-\frac{2}{1.2}-\frac{1}{3.4}\right] \\
+\left(\frac{1.3}{2.4}\right)^{2} \frac{5}{6} k^{\prime} 6\left[\log \left(\frac{4}{k^{\prime}}\right)-\frac{2}{1.2}-\frac{2}{3.4}-\frac{1}{5.6}\right]+\ldots .
\end{gathered}
$$

From (4.32), we get the following estimates when $k^{\prime} \rightarrow 0$,

$$
\frac{x+r}{2 r}=\frac{\mathrm{E}}{\mathrm{K}}=\frac{1}{\log \left(\frac{1}{k^{\prime}}\right)}-\frac{\log 4}{\log ^{2}\left(\frac{1}{k^{\prime}}\right)}+o\left(\frac{1}{\log ^{2}\left(\frac{1}{k^{\prime}}\right)}\right) .
$$

Now, we observe that $z$ can be written

$$
6 z=\frac{1-2 k^{\prime 2}}{\mathrm{E}^{2}} r^{3}\left(\frac{\mathrm{E}}{\mathrm{K}}\right)^{3}+\frac{r^{3} k^{\prime 2}}{\mathrm{~K}^{2}},
$$

hence we get

$$
6 z=r^{3} \frac{1-2 k^{\prime 2}}{\mathrm{E}^{2}}\left(\frac{x+r}{2 r}\right)^{3}+\frac{r^{3} k^{\prime 2}}{\mathrm{~K}^{2}}
$$

and this gives us the basic estimate

$$
6 z-r^{3}\left(\frac{x+r}{2 r}\right)^{3}=-\frac{3 r^{3}}{2} \frac{k^{\prime 2}}{\log ^{3}\left(\frac{1}{k^{\prime}}\right)}+o\left(\frac{k^{\prime 2}}{\log ^{3}\left(\frac{1}{k^{\prime}}\right)}\right) .
$$

And from (4.36) we get when $k^{\prime} \rightarrow 0$

$$
k^{\prime}=\mathrm{e}^{-\frac{2 r}{x+r}} 4(1+o(1))
$$

and we obtain when $k^{\prime} \rightarrow 0$

$$
6 z-r^{3} X^{3}=-24 r^{3} X^{3} \mathrm{e}^{-\frac{2}{X}}+o\left(X^{3} \mathrm{e}^{-\frac{2}{X}}\right)
$$

where $X=\frac{x+r}{2 r}$. Hence we have: 
Proposition 4.15. When $k^{\prime} \rightarrow 0$, the graph of $c$ is given by

$$
z=\frac{r^{3}}{6}\left(\frac{x+r}{2 r}\right)^{3}+F\left(\frac{x+r}{2 r}\right)
$$

where $F$ is a flat function of the form $F(X)=-4 r^{3} X^{3} \mathrm{e}^{-\frac{2}{X}}+o\left(X^{3} \mathrm{e}^{-\frac{2}{X}}\right)$. THEOREM 4.16. The intersection of the sphere $S(0, r), r>0$ with Martinet surface is not sub-analytic.

Proof. The intersection of the sphere $S(0, r)$ with the plane $y=0$ is formed by the union of the curve $c$ which is located in $z>0$, the symmetric curve $-c$ and the two points $x= \pm r, z=0$, which correspond to the intersections of the geodesic line $x=t, y=z=0$ with the sphere. Recall that this line is the projection of an abnormal bi-extremal. These two points are in the closure of $c$. Hence the intersection will form a closed curve around 0 .

The closure of $c$ is not semi-analytic at $(-r, 0)$, because at this point the graph of $c$ is the sum of an algebraic function and a flat function of order $X^{3} \mathrm{e}^{-\frac{2}{X}}$. Hence this curve is not sub-analytic, semi-analytic and subanalytic being the same in the plane. Since the intersection of $S(0, r)$ with the analytic set $y=0$ is not sub-analytic the sphere is not sub-analytic. The same is true for the distance function.

4.9.2. Parametrization of the sphere. To get the parametrization of the sphere of radius $r>0$, it is sufficient to set $t=r, u=K+r \sqrt{\lambda}$ and to impose the constraint $u \leq 3 K$ in the formulas of proposition 4.3 . It is represented on the following figures. The first one represents the intersection of the cut locus with the sphere of radius $r=0.1$ for $z>0$, the complete curve is the union of this one and of the curve obtained by the symmetry $(x, z) \mapsto(-x,-z)$. The second figure is the projection of the sphere on the plane $O x z$.

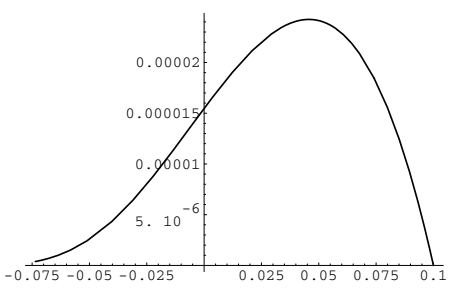

Figure 2

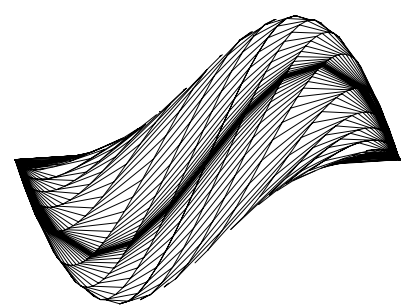

Figure 3 


\subsection{Intersection of the Wave front $W(0, r)$ with the Martinet PLANE}

Similarly we can compute the intersection of the wave front with the Martinet plane $y=0$. It is sufficient to plug $t=r=2 N \mathrm{~K} / \sqrt{\lambda}$ in the parametrization from proposition 4.3 which corresponds to the $N$-th intersection of the geodesic with the Martinet surface. For each $N$, we obtain a curve $c_{N}$ which admits the following parametric representation

$$
\begin{aligned}
x_{N}(k) & =-r+2 r \frac{E}{K} \\
z_{N}(k) & =\frac{r^{3}}{6 N^{2} K^{3}}\left[\left(2 k^{2}-1\right) E+k^{\prime 2} K\right]
\end{aligned}
$$

where $k \in] 0,1\left[\right.$, and the curve deduced from $c_{N}$ using the symmetry $(x, z) \rightarrow$ $(-x,-z)$. For $N=1$, we get the curve $c$ computed in 4.9 , which represents the intersection of the sphere with the Martinet plane. We represent the curves $c_{N}$ for $N=1, \ldots, 5$, on figure 4 . As previously we can evaluate the graphs of $c_{N}$ when $k \rightarrow 0$ or $k \rightarrow 1$. We summarize these results in the following proposition.

Proposition 4.17. The intersection of the wave front $W(0, r)$ with the Martinet plane $y=0$, and the half space $z>0$ is the union of curves $c_{N}, N \in \mathbb{N}^{*}$, whose closure admits two ramified point at $x= \pm r, z=0$. The graph of $c_{N}$ at $x=-r, z=0$ is given by

$$
z=\frac{r^{3}}{6 N^{2}}\left(\frac{x+r}{2 r}\right)^{3}+F\left(\frac{x+r}{2 r}\right),
$$

where $F(X)=\alpha r^{3} X^{3} \mathrm{e}^{-\frac{2}{X}}+o\left(X^{3} \mathrm{e}^{-\frac{2}{X}}\right), \alpha \neq 0$, and at $x=r, z=0$ by

$$
z=-\frac{2 r^{2}}{3 N^{2} \pi^{2}}(x-r)+o(x-r) \text {. }
$$

The exterior curve obtained for $N=1$ represents the intersection of the sphere with the Martinet plane.

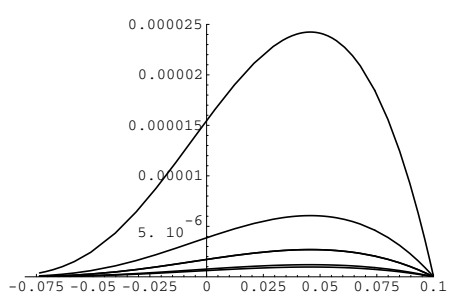

Figure 4

\subsection{EXP-LOG CATEGORY}

Since the intersection of the sphere $S(0, r)$ with the Martinet plane $y=0$ is not sub-analytic, an important question is to find the precise transcendence of this object. A first observation is the fact that in the domain $z>0$ it is the parametric curve $k \mapsto(x(k), z(k))$ where $k \in] 0,1$ [ and $x, z$ are defined by $(4.29),(4.30)$. It can be extended by continuity in the domain $z \geq 0$ by 
taking $k \in[0,1]$ and this curve is semi-analytic if $k \neq 1$. Near $k=1$ if we set $X_{1}=k^{\prime}$ and $X_{2}=\left(\log \frac{4}{k^{\prime}}\right)^{-1}$ where $k^{\prime} \in[0,1]$ and $k^{\prime 2}+k^{2}=1$, this curve is the image by an analytic mapping defined on a neighborhood of $O_{\mathbb{R}^{2}}$ into $\mathbb{R}^{2}$ of the pfaffian set $X_{1}=4 \mathrm{e}^{-\frac{1}{X_{2}}}$. We shall give a more precise description using the exp-log category, [16],[27].

THEOREM 4.18. The intersection of the sphere $S(0, r), r>0$ with the Martinet plane $y=0$ in the domain $z \geq 0$ is near $X=0$ with $X=\frac{x+r}{2 r}$ a graph of the form:

$$
z=F\left(X, \frac{\mathrm{e}^{-\frac{1}{X}}}{X^{2}}\right)
$$

where $X \geq 0$ and $F$ is an analytic mapping from a neighborhood of $O_{\mathbb{R}^{2}}$ into $\mathbb{R}$.

Proof. We can write near $k^{\prime}=0$ :

$$
X=\frac{x+r}{2 r}=\frac{E}{K}
$$

where

$$
\begin{aligned}
E\left(k^{\prime}\right) & =u_{1}\left(k^{\prime}\right) \log \frac{4}{k^{\prime}}+u_{2}\left(k^{\prime}\right) \\
K\left(k^{\prime}\right) & =u_{3}\left(k^{\prime}\right) \log \frac{4}{k^{\prime}}+u_{4}\left(k^{\prime}\right),
\end{aligned}
$$

the $u_{i}^{\prime} \mathrm{s}$ being analytic functions and:

$$
\begin{aligned}
& u_{1}\left(k^{\prime}\right)=\frac{k^{\prime 2}}{4}+o\left(k^{\prime 3}\right), u_{2}\left(k^{\prime}\right)=1-\frac{k^{\prime 2}}{8}+o\left(k^{\prime 3}\right), \\
& u_{3}\left(k^{\prime}\right)=1+\frac{k^{\prime 2}}{4}+o\left(k^{\prime 3}\right), u_{4}\left(k^{\prime}\right)=-\frac{k^{\prime 2}}{4}+o\left(k^{\prime 3}\right) .
\end{aligned}
$$

If we set: $X_{1}=k^{\prime}, X_{2}=\left(\log \frac{4}{k^{\prime}}\right)^{-1}$, we get that $X$ is the image of $X_{2}$ by the transformation:

$$
\begin{aligned}
X & =\frac{u_{1}\left(X_{1}\right)+X_{2} u_{2}\left(X_{1}\right)}{u_{3}\left(X_{1}\right)+X_{2} u_{4}\left(X_{1}\right)} \\
& =\left[\left(1-\frac{X_{1}^{2}}{8}+\ldots\right) X_{2}+\left(\frac{X_{1}^{2}}{4}+\ldots\right)\right]\left[1-\frac{X_{1}^{2}}{4}+\frac{X_{1}^{2}}{4} X_{2}+\ldots\right]
\end{aligned}
$$

and $X$ can be written as

$$
X=A_{1}\left(X_{1}, X_{2}\right)
$$

where $A_{1}$ is analytic near 0 . Moreover computing using (4.42) we get when $k^{\prime} \rightarrow 0:$

$$
X_{2} \sim X, X_{1} \sim 4 \mathrm{e}^{-\frac{1}{X}}
$$

and we can write:

$$
X_{2}=X\left(1+Y_{2}(X)\right), X_{1}=4 \mathrm{e}^{-\frac{1}{X}}\left(1+Y_{1}(X)\right)
$$

where $Y_{1}, Y_{2} \rightarrow 0$ when $X \rightarrow 0$. We can easily evaluate $Y_{1}$ and $Y_{2}$. Indeed inverting (4.42) we obtain:

$$
X_{2}=\frac{-u_{3} X+u_{1}}{u_{4} X-u_{2}}=X\left(1+\frac{O\left(X_{1}^{2}\right)}{X}+O\left(X_{1}^{2}\right)+\ldots\right) .
$$

Esaim: Cocv, December 1997, Vol. 2, PP. $377-448$ 
And since $X_{1} \sim 4 \mathrm{e}^{-\frac{1}{X}}$ we get that $Y_{2}$ is of order $X^{-1} \mathrm{e}^{-\frac{2}{X}}$. In particular $\frac{Y_{2}}{X} \rightarrow 0$ when $X \rightarrow 0$.

Moreover $Y_{1}$ and $Y_{2}$ are related as follows:

$$
\begin{aligned}
X_{1} & =4 \mathrm{e}^{-\frac{1}{X}}\left(1+Y_{1}(X)\right)=4 \mathrm{e}^{-\frac{1}{X\left(1+Y_{2}(X)\right)}} \\
& =4 \mathrm{e}^{-\frac{1}{X}} \mathrm{e}^{\left(-\frac{1}{X\left(1+Y_{2}(X)\right)}+\frac{1}{X}\right)}=4 \mathrm{e}^{-\frac{1}{X}} \mathrm{e}^{\left(\frac{Y_{2}}{X}-\frac{Y_{2}^{2}}{X}+\ldots\right)} .
\end{aligned}
$$

Therefore we have:

$$
Y_{1}(X)=A_{2}\left(\frac{Y_{2}}{X}, X\right)
$$

where $A_{2}$ is analytic near 0 and moreover $Y_{1} \sim \frac{Y_{2}}{X}$ when $X \rightarrow 0$. Inverting the previous equation we get that

$$
Y_{2}(X)=X A_{3}\left(X, Y_{1}\right)
$$

where $A_{3}$ is analytic at 0 and $Y_{2} \sim X Y_{1}$ when $X \rightarrow 0$.

From (4.42) we get:

$$
X=X_{2}+A_{4}\left(X_{1}, X_{2}\right)
$$

where $A_{4}\left(0, X_{2}\right)=0$. Hence equation (4.42) can be written

$$
X=X\left(1+Y_{2}+\frac{A_{4}\left(X_{1}, X_{2}\right)}{X}\right) \text {. }
$$

Using (4.45) and simplifying by $X^{2}$, we get that (4.42) is equivalent to

$$
0=A_{3}\left(X, Y_{1}\right)+\frac{A_{4}\left(X_{1}, X_{2}\right)}{X^{2}} .
$$

This equation can be written as:

$$
A_{5}\left(Y_{1}, X, \frac{\mathrm{e}^{-\frac{1}{X}}}{X^{2}}\right)=0
$$

where $A_{5}$ is analytic at $0, A_{5}(0)=0$ and $\frac{\partial A_{5}}{\partial Y_{1}}(0)=1$. Hence using the implicit function theorem in the analytic category we deduce that

$$
Y_{1}=A_{6}\left(X, \frac{\mathrm{e}^{-\frac{1}{X}}}{X^{2}}\right)
$$

where $A_{6}$ is analytic at 0 .

Therefore:

where $A_{7}$ is analytic at 0 .

$$
Y_{2}(X)=A_{7}\left(X, \frac{\mathrm{e}^{-\frac{1}{X}}}{X^{2}}\right)
$$

Now from $(4.30), z(k)$ is given by:

$$
z(k)=\frac{r^{3}}{6 K^{3}}\left[\left(2 k^{2}-1\right) E+k^{\prime 2} K\right]
$$

and is near $k^{\prime}=0$ an analytic function of $X_{1}=k^{\prime}$ and $X_{2}=\left(\log \frac{4}{k^{\prime}}\right)^{-1}$.

Hence in conclusion $z$ can be written near $X=0$ as:

$$
z=F\left(X, \frac{\mathrm{e}^{-\frac{1}{X}}}{X^{2}}\right)
$$

where $F$ is analytic at 0 . The theorem is then proved. 
REMARK 4.19. In the previous expansion, the variables $X$ and $X^{-2} \mathrm{e}^{-\frac{1}{X}}$ are called the scale. We can obtain a better scale if we use the fact that the $u_{i}^{\prime} \mathrm{s}$ are analytic with respect to $k^{\prime 2}$. An important question is to find the scale in the Martinet case when the set of geodesics is integrable by quadratures. It will be the object of a forthcoming article.

\section{One parameter deformation of the Martinet flat case}

In the previous section we have described in the flat case the singularities of the sub-Riemannian sphere and of the wave front due to the existence of abnormal bi-extremals. This example is a precious guide to understand in general the role of abnormal geodesics in sub-Riemannian geometry.

The aim of this section is to investigate the stability of the phenomena observed in the flat case and the accuracy of the flat case to describe the sub-Riemannian sphere in the Martinet case. A priori this stability is not obvious. Indeed in the flat case the geodesic lines contained in the Martinet surface are not strictly abnormal. Moreover the intersection of the conjugate and the cut locus from 0 is empty, but their closures intersect onto the geodesic line. To understand the general situation, we must use a gradated normal form of order 0 , as the one given in theorem 2.23 , which depends on 3 parameters. This model is too complicated for a preliminary study because it is not yet clear what are the adapted expansions needed to evaluate the exponential mapping. Hence we shall analyze in a first step a one-parameter deformation $\varepsilon \rightarrow d(\varepsilon)$ of the flat case. This model will be integrable by quadratures and we shall be able to evaluate the exponential mapping in a $C^{0}-$ neighborhood of the abnormal geodesic. It will give a clear geometric interpretation of the role of one parameter in the normal form, also it will allow to understand the general integrable case which shall be studied in [7].

\subsection{Construction of the model}

The one-parameter deformation will have the following properties.

5.1.1. Property. For each $\varepsilon \neq 0$, a geodesic is the projection of an unique bi-extremal considered as a curve in the projective space $P\left(T^{*} \mathbb{R}^{3}\right)$.

5.1.2. PRoperTy. The Hamiltonian differential equation whose solutions are the normal bi-extremals must have two cyclic coordinates and hence has to be integrable.

Having fixed such properties, the problem is to construct a one dimensional mechanical system of the form

$$
\dot{y}^{2}+P_{1}^{2}(y)=1,
$$

where $P_{1}^{2}$ is the potential depending on the parameter deformation $\varepsilon$ and two arbitrary constants $p_{x}$ and $p_{z}$. In order to satisfy the property 5.1.1, we must break the following symmetry occuring in the flat case: $P_{1}(-y)=P_{1}(y)$. Now to simplify the computations, we want to parametrize the set of normal geodesics using the lowest transcendence. This last constraint is very precise in terms of elliptic integrals and we have to make the following choice. The function $1-P_{1}^{2}(y)$ has to be a polynomial of degree 4 , which can be written Esaim: CoCV, DeCEMBER 1997, Vol. 2, PP. 377-448 
in canonical form as $\left(1-\eta^{2}\right)\left(\delta k^{\prime 2}+k^{2} \eta^{2}\right), \delta=0,1,-1$ where $\eta$ is deduced from $y$ using a translation combined with a similarity, but not a more general homographic transformation.

5.1.3. Model. We choose the one-form $\omega=(1+\varepsilon y) d z-\frac{1}{2} y^{2} d x$. The distribution $D=\operatorname{ker} \omega$ is spanned by

$$
F_{1}=(1+\varepsilon y) \frac{\partial}{\partial x}+\frac{y^{2}}{2} \frac{\partial}{\partial z}, \quad F_{2}=\frac{\partial}{\partial y} .
$$

The brackets are given by

$$
\left[F_{1}, F_{2}\right]=\varepsilon \frac{\partial}{\partial x}+y \frac{\partial}{\partial z}, \quad\left[\left[F_{1}, F_{2}\right], F_{2}\right]=\frac{\partial}{\partial z}, \quad\left[\left[F_{1}, F_{2}\right], F_{1}\right]=0
$$

and the brackets of length $\geq 4$ are 0 . Hence the Lie algebra generated by $\left\{F_{1}, F_{2}\right\}$ is nilpotent. The singular set $S: \operatorname{det}\left(F_{1}, F_{2},\left[F_{1}, F_{2}\right]\right)=0$, where $\omega$ is not a contact form is given by $(1+\varepsilon y / 2)=0$. Therefore if $\varepsilon \neq 0, S$ is the union of the two planes: $y=0$ and $y=-2 / \varepsilon$. We shall localize our study in a neighborhood of 0 , hence we assume first

$$
|y \varepsilon|<2 \text {. }
$$

In this domain the Martinet surface is the plane $y=0$.

We choose the metric $g$ by taking $F_{1}$ and $F_{2}$ as orthonormal vector fields. If $(1+\varepsilon y) \neq 0$, we can write on $D, d z=\frac{y^{2}}{2(1+\varepsilon y)} d x$. Hence we shall restrict our study to the domain $U$ given by

$$
|y \varepsilon|<1 \text {, }
$$

where the metric can be represented by

$$
g=\frac{1}{(1+\varepsilon y)^{2}} d x^{2}+d y^{2},
$$

and $\widetilde{g}$ will denote the induced Riemannian metric on the plane $(x, y)$.

\subsection{Equations of the set of geodesics in the domain $U$}

5.2.1. Abnormal geodesics. The system is written

$$
\begin{aligned}
\dot{x} & =u(1+\varepsilon y) \\
\dot{y} & =v \\
\dot{z} & =u \frac{y^{2}}{2} .
\end{aligned}
$$

The control corresponding to abnormal geodesics is $v \equiv 0$ and the abnormal geodesics are contained in the Martinet plane $y=0$ and they satisfy the equations

$$
\dot{x}=u, \quad y=0, \quad z=z_{0} .
$$

We shall denote $\gamma: t \longrightarrow( \pm t, 0,0)$ the abnormal geodesics starting from 0 and parametrized by arc-length. 
5.2.2. Normal geodesics. We set $F_{3}=\frac{\partial}{\partial z}$ and $P_{i}=\left\langle p, F_{i}(q)\right\rangle, i=1,2,3$. The Hamiltonian corresponding to normal bi-extremals is

$$
H_{n}=\frac{1}{2}\left(P_{1}^{2}+P_{2}^{2}\right)
$$

where

$$
P_{1}=p_{x}(1+\varepsilon y)+p_{z} \frac{y^{2}}{2}, \quad P_{2}=p_{y} .
$$

There exists two cyclic coordinates $x, z$ and $p_{x}, p_{z}$ are first integrals. The normal bi-extremals are solutions of

$$
\begin{aligned}
\dot{x} & =(1+\varepsilon y)\left[p_{x}(1+\varepsilon y)+p_{z} \frac{y^{2}}{2}\right] \\
\dot{y} & =p_{y} \\
\dot{z} & =\frac{y^{2}}{2}\left[p_{x}(1+\varepsilon y)+p_{z} \frac{y^{2}}{2}\right] \\
\dot{p}_{x} & =0 \\
\dot{p}_{y} & =-\left(\varepsilon p_{x}+p_{z} y\right)\left[p_{x}(1+\varepsilon y)+p_{z} \frac{y^{2}}{2}\right] \\
\dot{p}_{z} & =0 .
\end{aligned}
$$

In the coordinates $(q, P)$ we have

$$
\begin{aligned}
\dot{x} & =(1+\varepsilon y) P_{1} \\
\dot{y} & =P_{2} \\
\dot{z} & =\frac{y^{2}}{2} P_{1} \\
\dot{P}_{i} & =\sum_{j=1}^{2}\left\{P_{i}, P_{j}\right\} P_{j},
\end{aligned}
$$

and computing we get

$$
\left\{P_{1}, P_{2}\right\}=\left\langle p, \varepsilon \frac{\partial}{\partial x}+y \frac{\partial}{\partial z}\right\rangle
$$

Using for $|y \varepsilon|<1$,

$$
\frac{\partial}{\partial x}=\frac{1}{(1+\varepsilon y)}\left(F_{1}-\frac{y^{2}}{2} F_{3}\right),
$$

we obtain

$$
\left\{P_{1}, P_{2}\right\}=y P_{3}+\frac{\varepsilon}{1+\varepsilon y}\left(P_{1}-\frac{y^{2}}{2} P_{3}\right)
$$

and we get the equations

$$
\begin{aligned}
& \dot{P}_{1}=\left[y P_{3}+\frac{\varepsilon}{1+\varepsilon y}\left(P_{1}-\frac{y^{2}}{2} P_{3}\right)\right] P_{2} \\
& \dot{P}_{2}=-\left[y P_{3}+\frac{\varepsilon}{1+\varepsilon y}\left(P_{1}-\frac{y^{2}}{2} P_{3}\right)\right] P_{1} \\
& \dot{P}_{3}=0 .
\end{aligned}
$$

The mechanical interpretation is the following. We set $P_{1}=\cos \theta, P_{2}=$ $\sin \theta$. Hence if $\theta \neq n \pi$, we have $\dot{\theta}=-\left(p_{x} \varepsilon+p_{z} y\right)$. Therefore $\ddot{\theta}+\lambda \sin \theta=0$ where $\lambda=p_{z}$. It is the pendulum equation with $\dot{\theta}(0)=-p_{x} \varepsilon$. 


\subsection{Geodesics of the induced metric}

By setting $P_{3}=0$ in the previous equations and if we let

$$
\widetilde{F}_{1}=(1+\varepsilon y) \frac{\partial}{\partial x}, \quad \widetilde{F}_{2}=\frac{\partial}{\partial y},
$$

and

$$
\begin{gathered}
\widetilde{P}_{1}=p_{x}(1+\varepsilon y), \quad \widetilde{P}_{2}=p_{y}, \\
\widetilde{H}=\frac{1}{2}\left(\widetilde{P}_{1}^{2}+\widetilde{P}_{2}^{2}\right),
\end{gathered}
$$

the equations of the geodesics of the induced metric $\tilde{g}$ are then

$$
\begin{array}{ll}
\dot{x}=(1+\varepsilon y) \widetilde{P}_{1} & \dot{\widetilde{P}}_{1}=\frac{\varepsilon}{1+\varepsilon y} \widetilde{P}_{1} \widetilde{P}_{2} \\
\dot{y}=\widetilde{P}_{2} & \dot{\tilde{P}}_{2}=-\frac{\varepsilon}{1+\varepsilon y} \widetilde{P}_{1}^{2} .
\end{array}
$$

They can be studied by considering the equation

$$
\dot{y}^{2}+\widetilde{P}_{1}^{2}=1
$$

where $\widetilde{P}_{1}=p_{x}(1+\varepsilon y)$. It is a mechanical system whose physical space is $\left|\widetilde{P}_{1}(y)\right| \leq 1$. At $t=0$, one has $y(0)=0$, hence we get the condition $\left|p_{x}\right| \leq 1$. The change of sign of $\dot{y}$ are given by solving $\widetilde{P}_{1}= \pm 1$, i.e., $p_{x}(1+\varepsilon y)= \pm 1$. We can assume $\varepsilon \neq 0$ and we get $y=\frac{ \pm 1-p_{x}}{\varepsilon p_{x}}$ if $p_{x} \neq 0$.

The solutions can be easily studied by considering the graph of $\widetilde{P}_{1}$. If $p_{x} \neq 0$, they are precisely two intersections of $\widetilde{P}_{1}$ with \pm 1 and they are denoted $y_{-}<y_{+}$. If $p_{x} \neq \pm 1$, one has $y_{-}<0<y_{+}$and the system oscillates between $y_{-}$and $y_{+}$. If $p_{x}= \pm 1, \dot{y}(0)=0$ and $\ddot{y}(0)=-\varepsilon$, and $y_{-}$ or $y_{+}$is 0 . If $p_{x}=0$, there is no intersection of $\widetilde{P}_{1}$ with \pm 1 and there is no oscillation. More precisely the solution can be computed. Indeed we have

$$
\ddot{y}=\dot{\widetilde{P}}_{2}=-\frac{\varepsilon}{1+\varepsilon y} \widetilde{P}_{1}^{2}
$$

and we get the equation

$$
\ddot{y}+\varepsilon p_{x}^{2}(1+\varepsilon y)=0
$$

which can be easily integrated.

The optimality status of the geodesics for the metric $\widetilde{g}$ can be studied. In particular, we can evaluate the conjugate and the cut loci. Using index theory we can analyze the conjugate locus for the sub-Riemannian geodesics corresponding to $p_{z}=0$ which project onto geodesics for $\widetilde{g}$. An interesting result in this direction is given by the following proposition.

Proposition 5.1. The geodesic line parametrized by arc-length $\bar{\gamma}: x=$ $z=0, y= \pm t$ is the only geodesic parametrized by arc-length $e:[0, T] \rightarrow U$, $e=(x, y, z)$ such that $e(0)=0, e(T)=(0, *, 0)$. In particular $\bar{\gamma}$ is isolated in the set of geodesics.

Proof. Observe that for a normal bi-extremal

$$
\frac{d}{d t}\left(p_{x} x+p_{z} z\right)=P_{1}^{2} \text {. }
$$


Hence if $x(0)=z(0)=0$, we have

$$
p_{x} x(T)+p_{z} z(T)=\int_{0}^{T} P_{1}^{2}(t) d t .
$$

Therefore if $P_{1} \neq 0$, we have

$$
p_{x} x(T)+p_{z} z(T)>0 .
$$

In particular if $p_{x}=P_{1}(0) \neq 0$, we cannot have both $x(T)=z(T)=0$. If $p_{x}=0, P_{1} \neq 0$ then $p_{z} \neq 0$ and $z(T) \neq 0$. If $p_{x}=0, P_{1}=0$, we have $p_{z}=0$ if $y(t) \neq 0$. Hence the line $x=z=0$ corresponding to $p_{x}=p_{z}=0$ is isolated.

\subsection{Normal geOdesics CORRESPOnding TO $p_{z} \neq 0$}

5.4.1. Symmetry. We introduce the following parameters: $P_{1}(0)=p_{x}=$ $\sin \varphi, P_{2}(0)=\cos \varphi$, where $\varphi \in\left[0,2 \pi\left[\right.\right.$ and $p_{z}=\lambda$. Observe that equations (5.1) are left invariant for the following transformation: $X=-x, Y=y$, $Z=-z, P_{X}=-p_{x}, P_{Y}=p_{y}, P_{Z}=-p_{z}$. Hence the set of normal geodesics is left invariant by the symmetry: $S_{2}:(x, y, z) \rightarrow(-x, y,-z)$ and the same is true for the metric. Therefore in our study, we can assume $\lambda>0$. Also equations (5.1) and the metric are left invariant by the transformation $\left(y, p_{y}, \varepsilon\right) \rightarrow\left(-y,-p_{y},-\varepsilon\right)$. Hence in our study we can fix the sign of $\varepsilon$ and for convenience we shall assume $\varepsilon \leq 0$.

5.4.2. Characteristic Equation in normal Form. We analyze now the equations defining normal bi-extremals parametrized by arc-length: $H_{n}=$ $1 / 2$ and we get

$$
\dot{y}^{2}+P_{1}^{2}(y)=1,
$$

where $P_{1}=p_{x}(1+\varepsilon y)+p_{z} y^{2} / 2$ and $p_{x}, p_{z}$ are constant, $p_{x}=\sin \varphi, p_{z}=$ $\lambda>0$.

The physical space is $\left\{y \in U ;\left|P_{1}(y)\right| \leq 1\right\}$. Hence we get the condition $\left(H_{3}\right)$

$$
\left|p_{x}\right| \leq 1 \text {. }
$$

We can write

$$
1-P_{1}=1-p_{x}+\frac{\varepsilon^{2} p_{x}^{2}}{2 \lambda}-\frac{\lambda}{2}\left(y+\frac{\varepsilon p_{x}}{\lambda}\right)^{2} .
$$

Since $\left|p_{x}\right| \leq 1$, we have $1-p_{x}+\frac{\varepsilon^{2} p_{x}^{2}}{2 \lambda}>0$ when $\varepsilon \neq 0$. If $\varepsilon=0$, our previous study shows that we can assume $\left|p_{x}\right| \neq 1$. Hence if we set

$$
2 k^{2}=1-p_{x}+\frac{\varepsilon^{2} p_{x}^{2}}{2 \lambda},
$$

we can always assume $2 k^{2}>0$.

Now we can write

$$
1+P_{1}=1+p_{x}-\frac{\varepsilon^{2} p_{x}^{2}}{2 \lambda}+\frac{\lambda}{2}\left(y+\frac{\varepsilon p_{x}}{\lambda}\right)^{2} .
$$

We introduce

$$
2 k^{\prime \prime}=1+p_{x}-\frac{\varepsilon^{2} p_{x}^{2}}{2 \lambda}
$$


and we have $k^{2}+k^{\prime \prime}=1$. Let $k^{\prime 2}=\left|k^{\prime \prime}\right|$, we can write $k^{\prime \prime}=\delta k^{\prime 2}$, where $\delta$ is a constant equals to $0,1,-1$. Hence equation (5.6) can be written in the normal form

$$
\dot{y}^{2}=4 k^{2}\left(1-\eta^{2}\right)\left(k^{\prime \prime}+k^{2} \eta^{2}\right)
$$

where we have set

$$
\eta=\frac{1}{2 k}\left(y \sqrt{\lambda}+\frac{\varepsilon p_{x}}{\sqrt{\lambda}}\right), \quad k>0
$$

and endly the equation which has to be integrated is

$$
\frac{\dot{\eta}^{2}}{\lambda}=\left(1-\eta^{2}\right)\left(k^{\prime \prime}+k^{2} \eta^{2}\right)
$$

\subsection{Form of the potential $P_{1}^{2}$}

We can analyze the behaviors of the trajectories of the characteristic equation (5.6) by considering the graph of $P_{1}$ only and its intersections with the lines $P_{1}= \pm 1$. Observe that

$$
P_{1}=p_{x}(1+\varepsilon y)+\lambda \frac{y^{2}}{2}
$$

and $P_{1}(0)=p_{x}$, where $\left|p_{x}\right| \leq 1$. When $y \rightarrow \infty, P_{1} \rightarrow+\infty$ and $P_{1}$ has an unique minimum $m$ which satisfies $P_{1}^{\prime}(m)=0$, i.e., $m=-\frac{\varepsilon p_{x}}{\lambda}$ and we have

$$
P_{1}(m)=p_{x}-\frac{\varepsilon^{2} p_{x}^{2}}{2 \lambda} .
$$

We denote by $y_{1}<y_{2}$ the solutions of the equation $P_{1}(y)=1$, i.e., the roots of :

$$
\frac{\lambda y^{2}}{2}+\varepsilon p_{x} y+p_{x}-1=0
$$

and $y_{1} y_{2}=2\left(p_{x}-1\right) / \lambda$. If $p_{x}=1$ and $\varepsilon<0$ we are in the limit case $y_{1}=0$, $y_{2}=-2 \varepsilon / \lambda>0$. Moreover $P_{2}(0)=0, \dot{y}(0)=P_{2}(0)=0, \ddot{y}(0)=\dot{P}_{2}(0)=$ $-\varepsilon P_{1}^{2}(0)>0$. From this analysis we deduce the following.

Lemma 5.2. Assume $\varepsilon<0$. The equation $P_{1}(y)=1$ has two distinct roots satisfying $y_{1}<y_{2}$ and $0 \in\left[y_{1}, y_{2}\right]$. The motion $y(t)$ with $y(0)=0$ is confined to the segment $\left[y_{1}, y_{2}\right]$. If $p_{x} \neq 1$ then $\left.0 \in\right] y_{1}, y_{2}\left[\right.$, if $p_{x}=1, y_{1}=0$.

Now we must compute the solution of $P_{1}(y)=-1$ which are the roots of

$$
\frac{\lambda y^{2}}{2}+\varepsilon p_{x} y+p_{x}+1=0
$$

The discriminant is $-4 \lambda k^{\prime \prime}$ and there is a critical value when $P_{1}(m)=-1$ which corresponds to the case $k^{\prime \prime}=0$. Hence we must distinguish between three cases.

Case A: $k^{\prime \prime}>0$ and there is no real root.

Case B: $k^{\prime \prime}<0$ and we have two distincts roots $y_{3}<y_{4}$.

Case C: $k^{\prime \prime}=0$ which corresponds to the limit case: $y_{3}=y_{4}=m$.

We represent below the corresponding graphs of $P_{1}$. When $\varepsilon \neq 0$, we have the three cases and when $\varepsilon=0$ (flat case) we are always in case A. It has to be compared with the analysis in [23] where the potential $P_{1}^{2}$ is different but we are faced with the same discussion. The geometric explanation is the following. In case $C$, the motion of $y(t)$ is not periodic because the time 
we need to reach the position $m$ corresponding to a double root of $1-P_{1}^{2}(y)$ is infinite. If we interpret the motion in term of a pendulum it corresponds to a motion on a separatrix. In the flat case such a behavior corresponds to a normal geodesic not starting from 0 . Hence the role of the parameter $\varepsilon$ is to push such geodesics in the physical space.

Another consequence is the existence for $\varepsilon \neq 0$ of geodesics which correspond to a rotating pendulum.

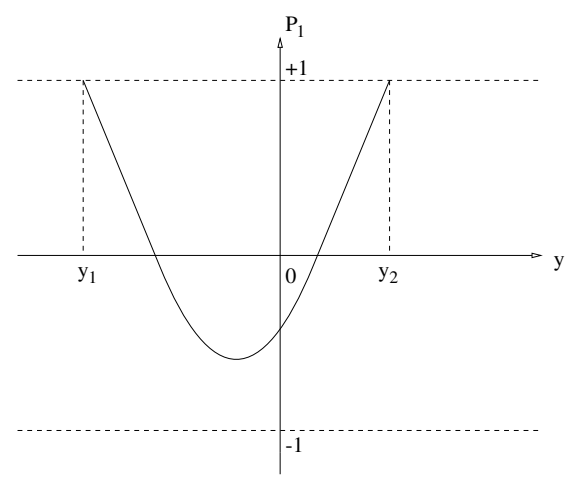

Figure 5. Case A

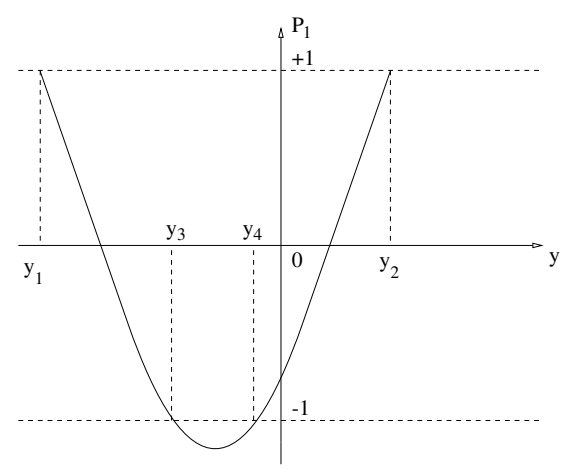

Figure 6. Case B

When $p_{x}=0$, there is no real solution to $P_{1}=-1$. Hence in case $\mathrm{B}$ and $\mathrm{C}$ we can assume $p_{x} \neq 0$. In case $\mathrm{B}$, we have $y_{3} y_{4}=2\left(p_{x}+1\right) / \lambda$. Therefore if $p_{x} \neq-1$ both roots have the same sign which is the sign of $p_{x}$. If $p_{x}=-1$, $y_{4}=0$ and $y_{3}=2 \varepsilon / \lambda<0$. In case $\mathrm{C}$, the root is $m=-\varepsilon p_{x} / \lambda$ and $m \neq 0$. If $p_{x}=-1, \dot{y}(0)=0$ and $\ddot{y}(0)=-\varepsilon>0$.

Lemma 5.3. Assume $\varepsilon<0$. If $k^{\prime \prime}<0$, the solutions of $P_{1}(y)=-1$ are two distinct points $y_{3}<y_{4}$. If $p_{x} \neq-1$, they are non zero and have both the sign of $p_{x}$. If $p_{x}=-1$, then $y_{4}=0$ and $y_{3}<0$. If $k^{\prime \prime}=0$, the root solution of $P_{1}(y)=-1$ is double and is given by $m=-\varepsilon p_{x} / \lambda$.

Esaim: CoCv, December 1997, Vol. 2, Pp. 377-448 


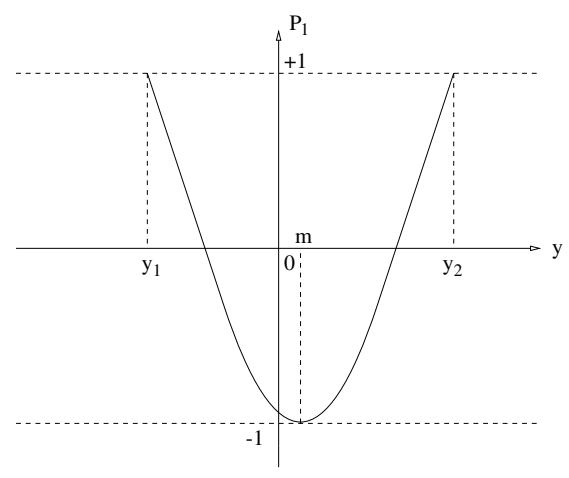

Figure 7. Case C

From our analysis we deduce the following result.

LEMMA 5.4. If $k^{\prime \prime} \neq 0$, the trajectory of $\dot{y}^{2}+P_{1}^{2}(y)=1$ oscillates periodically between $y_{-}<y_{+}$where $\left[y_{-}, y_{+}\right]$is the interval $\left[y_{1}, y_{2}\right]$ in case $A$, the interval $\left[y_{1}, y_{3}\right]$ or $\left[y_{4}, y_{2}\right]$ containing 0 in case $B$. When $k^{\prime \prime}=0$, the motion is not periodic.

We shall need the following lemma.

LEMMA 5.5. Assume $\varepsilon<0$. The critical set $k^{\prime \prime}=0$ is the trace of the graph of $\lambda=\frac{\varepsilon^{2} p_{x}^{2}}{2\left(1+p_{x}\right)}$ in the domain $\lambda>0, p_{x} \in[-1,+1]$ (see figure 8 ).

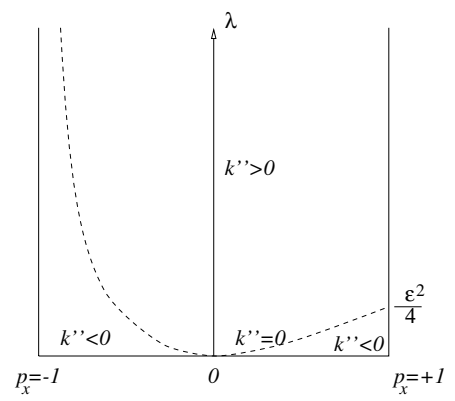

FiguRE 8

\subsection{Notation}

Our aim is to analyze the exponential mapping $\exp _{0}$ in a $C^{0}$-neighborhood of the reference abnormal geodesic $\gamma: t \rightarrow( \pm t, 0,0)$. We shall denote by $e: t \rightarrow(x(t), y(t), z(t)), t \in[0, T]$ a normal geodesic parametrized by arc-length and such that $e(0)=0$. From now on we shall assume that $\varepsilon<0$. 


\subsection{Parametrization of the Regular geodesics $(\lambda>0)$}

In cases $\mathrm{A}$ and $\mathrm{B}$, the $y$ coordinate oscillates periodically between $\left\{y_{-}, y_{+}\right\}$ and the period is given by $P=2 \tau$ where

$$
\tau=\int_{y_{-}}^{y_{+}} \frac{d y}{\sqrt{1-P_{1}^{2}(y)}} .
$$

Let $0<t_{1}<t_{2}<\cdots<t_{N} \leq T$ be the times such that $y\left(t_{i}\right)=0$. Define

$$
\sigma= \begin{cases}\operatorname{sign} \dot{y}(0) & \text { if } \dot{y}(0) \neq 0, \\ \operatorname{sign} \ddot{y}(0) & \text { if } \dot{y}(0)=0 .\end{cases}
$$

Observe that we cannot have $\dot{y}(0)=\ddot{y}(0)=0$, since $\dot{y}(0)=P_{2}(0)=\cos \varphi$ and $\ddot{y}(0)=-\varepsilon P_{1}^{2}(0)$. Hence for $\left.\varphi \in\right]-\frac{\pi}{2},+\frac{\pi}{2}[, \dot{y}(0)>0$ and for $\varphi \in] \frac{\pi}{2}, \frac{3 \pi}{2}[$, $\dot{y}(0)<0$. The limit cases correspond to $\varphi \in\left\{-\frac{\pi}{2},+\frac{\pi}{2}\right\}$, where $\dot{y}(0)=0$ and $\ddot{y}(0)>0$.

To compute the normal geodesics we must distinguish the case $\sigma=1$ and $\sigma=-1$. A first method is to parametrize the trajectories by $y$ instead of $t$. This method is a general method which can be used in the integrable case. In particular to evaluate the trace of the wave front with the Martinet surface, we set $y(T)=0$ and we get the following formulas.

For $N=2 p+1$ :

$$
\begin{aligned}
& x(T)=2 \int_{0}^{y_{\sigma}} \frac{\sigma(1+\varepsilon y) P_{1}(y) d y}{\sqrt{1-P_{1}^{2}(y)}}+(N-1) \int_{y_{-}}^{y_{+}} \frac{(1+\varepsilon y) P_{1}(y) d y}{\sqrt{1-P_{1}^{2}(y)}} \\
& z(T)=\int_{0}^{y_{\sigma}} \frac{\sigma y^{2} P_{1}(y) d y}{\sqrt{1-P_{1}^{2}(y)}}+(N-1) \int_{y_{-}}^{y_{+}} \frac{y^{2} P_{1}(y) d y}{2 \sqrt{1-P_{1}^{2}(y)}} .
\end{aligned}
$$

For $N=2 p$ :

$$
\begin{aligned}
& x(T)=N \int_{y_{-}}^{y_{+}} \frac{(1+\varepsilon y) P_{1}(y) d y}{\sqrt{1-P_{1}^{2}(y)}} \\
& z(T)=N \int_{y_{-}}^{y_{+}} \frac{y^{2} P_{1}(y) d y}{2 \sqrt{1-P_{1}^{2}(y)}} .
\end{aligned}
$$

We can compute the integrals using the $\eta$ coordinate

$$
\eta=\frac{1}{2 k}\left(y \sqrt{\lambda}+\frac{\varepsilon p_{x}}{\sqrt{\lambda}}\right)
$$

which implies

$$
\begin{aligned}
\eta(0) & =\frac{\varepsilon p_{x}}{2 k \sqrt{\lambda}} \\
y & =\frac{1}{\sqrt{\lambda}}\left(2 k \eta-\frac{\varepsilon p_{x}}{\sqrt{\lambda}}\right) \\
1-P_{1}^{2}(y) & =4 k^{2}\left(1-\eta^{2}\right)\left(k^{\prime \prime}+k^{2} \eta^{2}\right) \\
P_{1}(y) & =2 k^{2} \eta^{2}+p_{x}-\frac{\varepsilon^{2} p_{x}^{2}}{2 \lambda} .
\end{aligned}
$$

Let $\eta_{+}, \eta_{-}$be the respective images of $y_{+}, y_{-}$, if we set

$$
Z^{\sigma}=\int_{0}^{y_{\sigma}} \frac{\sigma y^{2} P_{1}(y) d y}{\sqrt{1-P_{1}^{2}(y)}},
$$


we get

$$
Z^{\sigma}=\frac{1}{\lambda^{3 / 2}} \int_{\eta(0)}^{\eta_{\sigma}} \frac{\sigma\left(2 k \eta-\frac{\varepsilon p_{x}}{\sqrt{\lambda}}\right)^{2}\left(2 k^{2} \eta^{2}+p_{x}-\frac{\varepsilon^{2} p_{x}^{2}}{2 \lambda}\right) d \eta}{\sqrt{\left(1-\eta^{2}\right)\left(k^{\prime \prime}+k^{2} \eta^{2}\right)}}
$$

The length of the trajectory between the first two zeros of $y$ is given by

$$
\theta^{\sigma}=\frac{2}{\sqrt{\lambda}} \int_{\eta(0)}^{\eta_{\sigma}} \frac{\sigma d \eta}{\sqrt{\left(1-\eta^{2}\right)\left(k^{\prime \prime}+k^{2} \eta^{2}\right)}} .
$$

Corollary 5.6. If $k^{\prime \prime} \neq 0, p_{x} \neq \pm 1$, a geodesic of length $L(e)>P$ (period) is not a minimizer.

Proof. Fix $\lambda$ and $p_{x} \neq \pm 1$, the above formulas show that the geodesic corresponding to $\sigma=+1$ and the geodesic corresponding to $\sigma=-1$ intersect for $t=P$ on $y=0$. Hence using lemma 4.13 they are not length minimizing beyond this point.

\subsection{Assumptions}

The quantity $Z^{\sigma}$ represents the drift of the $z$ variable corresponding to the first two zeros of $y$. We shall estimate this quantity. We make the following assumptions:

$$
T \leq M
$$

where $T$ is the length of $e$, i.e., we assume that the length is uniformly bounded.

$$
|\varepsilon| \leq M^{\prime}
$$

\subsection{IsOlated ABNORMAL GEODESiC}

Definition 5.7. The abnormal geodesic $\gamma: t \mapsto( \pm t, 0,0)$ is said to be isolated (resp. $C^{0}$-isolated) if there exists no normal geodesic $e:[0, T] \mapsto U$ (resp. contained in a $C^{0}$-neighborhood of $\gamma$ ) distinct of $\gamma$ such that $e(0)=0$, $e(T) \in \operatorname{Im} \gamma$.

The isolation of $\gamma$ is a basic property which has to be studied. First, we have:

Lemma 5.8. In the flat case $\gamma$ is isolated.

Proof. See the wave front represented on figure 4.

In the general case we have the following lemmas.

LEMMA 5.9. Let $e$ be a normal geodesic distinct of $\gamma$ such that $e(0)=0$ and $e(T) \in \operatorname{Im} \gamma$. Then we must have $p_{x}<0$.

Proof. We have $z(T)=\int_{0}^{T} \frac{y^{2}}{2} P_{1}(y) d t$ where $y(t) \neq 0$ and $P_{1} \neq 0$. If $z(T)=$ 0 , then $P_{1}$ must change of sign on $U$. Since $P_{1}(y)=p_{x}(1+\varepsilon y)+p_{z} y^{2} / 2$, if $p_{x} p_{z}=0$, the $\operatorname{sign}$ is constant. Hence we can assume $p_{x} p_{z} \neq 0$ and we must have $p_{x} p_{z}<0$.

Lemma 5.10. Let us assume that $p_{x}<0$ and $\sigma=-1$. Then in case $B$, we have $Z^{\sigma}<0$ 
Proof. In case $B$, if $p_{x}<0$ then both roots $y_{3}, y_{4}$ are negative and $y$ oscillates between $y_{4}$ and $y_{2}$. If $\sigma=-1$, then $y$ moves first from 0 to $y_{4}$ and $P_{1}(y)<0$ (see figure 6 ). Hence the drift $Z^{\sigma}$ is strictly negative.

LEMMA 5.11. Let e be a normal geodesic distinct of $\gamma$ such that $e(0)=0$ and $e(T) \in \operatorname{Im} \gamma$. Let $Y=\sup _{t \in[0, T]}|y(t)|$ and assume that $Y \leq 1 /\left(2 M^{\prime}\right)$. Then we must have $\left|p_{x}\right| \leq p_{z} Y^{2}$.

Proof. If $Y \leq 1 /\left(2 M^{\prime}\right)$, then $(1+\varepsilon y) \geq \frac{1}{2}$. If $z(0)=z(T)=0$, then there exists $t_{1} \in[0, T]$ such that

$$
p_{x}=-\frac{p_{z} y^{2}\left(t_{1}\right)}{2\left(1+\varepsilon y\left(t_{1}\right)\right)} .
$$

Hence, if $Y \leq \frac{1}{2 M^{\prime}}$

$$
\left|p_{x}\right| \leq \frac{p_{z} y^{2}\left(t_{1}\right)}{2\left|1+\varepsilon y\left(t_{1}\right)\right|} \leq p_{z} Y^{2}
$$

5.9.1. NOTATION. We shall denote by

$$
I_{1}=4 \int_{0}^{1} \frac{\eta\left(2 \eta^{2}-1\right)}{\sqrt{1-\eta^{2}}} d \eta=\frac{4}{3} .
$$

\subsection{Case A: estimation of the dRift $Z^{\sigma}$}

5.10.1. Notation. We shall denote by $d_{1}$ the drift $Z^{+}=Z^{-}$corresponding to the flat case $\varepsilon=0$ and $I_{2}=\lambda^{\frac{3}{2}} d_{1}$ the normalized drift. Using proposition 4.3, we have

$$
I_{2}=\frac{4}{3}\left[E\left(1-k^{\prime 2}\right)+k^{\prime 2}(K-E)\right] .
$$

Lemma 5.12. Let $I_{3}=\inf I_{2}$ for $p_{x} \leq 0$. Then $I_{3}>0$.

Proof. If $p_{x} \leq 0$, then $k \geq 1 / \sqrt{2}$. From [25] p. 74, $k \mapsto K(k)$ is an increasing function and

$$
K-E=k^{2} \int_{0}^{K} \operatorname{sn}^{2} u d u \geq \frac{1}{2} \int_{0}^{K\left(\frac{1}{\sqrt{2}}\right)} \operatorname{sn}^{2} u d u
$$

Hence

$$
I_{2} \geq \frac{2 k^{\prime 2}}{3} \int_{0}^{K\left(\frac{1}{\sqrt{2}}\right)} \operatorname{sn}^{2} u d u
$$

When $k^{\prime} \rightarrow 0, I_{2}$ can be estimated and we get

$$
I_{2}=I_{1}+O\left(k^{\prime 2} \log \left(\frac{1}{k^{\prime}}\right)\right) .
$$

Hence we have proved that $I_{3}>0$.

Proposition 5.13. Assume $k^{\prime \prime}>0, p_{x} \leq 0$. Then we have

$$
Z^{\sigma}\left(p_{x}\right)=\frac{1}{\lambda^{\frac{3}{2}}}\left[I_{2}\left(p_{x}\right)+O\left(\frac{1}{\sqrt{\lambda}}\right)\right]
$$

where $O\left(\frac{1}{\sqrt{\lambda}}\right) \rightarrow 0$ uniformly when $\lambda \rightarrow \infty$. 
Proof. We must compare at $p_{x}$ fixed the difference between $Z^{\sigma}$ and the drift corresponding to the flat case $\varepsilon=0$. We write

$$
k^{2}(\alpha)=\frac{1-p_{x}}{2}+\alpha, k^{\prime \prime}(\alpha)=\frac{1+p_{x}}{2}-\alpha,
$$

where $\alpha=\frac{\varepsilon^{2} p_{x}^{2}}{4 \lambda}$.

We set

$$
f(\alpha)=\left(k^{\prime \prime}(\alpha)+k^{2}(\alpha) \eta^{2}\right)^{-\frac{1}{2}}=\left[k^{\prime \prime}(0)+k^{2}(0) \eta^{2}+\alpha\left(\eta^{2}-1\right)\right]^{-\frac{1}{2}} .
$$

Hence for $\eta \in[-1,+1]$, we have

$$
f(\alpha) \geq f(c) \text { for } c \in[0, \alpha] .
$$

Since

$$
f^{\prime}(\alpha)=-\frac{1}{2}\left(\eta^{2}-1\right)\left(k^{\prime \prime}(\alpha)+k^{2}(\alpha) \eta^{2}\right)^{-\frac{3}{2}},
$$

we have for $c \in[0, \alpha], \eta \in[-1,+1]$,

$$
\left|f^{\prime}(c)\right| \leq \frac{K_{1}}{k^{2}(c) \eta^{2} \sqrt{k^{\prime \prime}(\alpha)+k^{2}(\alpha) \eta^{2}}}
$$

We shall estimate the drift $Z^{\sigma}, \sigma=+1$, the case $\sigma=-1$ can be analyzed similarly. We use the uniform bound given by $\left(H_{4}\right): \theta^{+} \leq M$, where

$$
\theta^{+}=\frac{2}{\sqrt{\lambda}} \int_{\eta(0)}^{1} \frac{d \eta}{\sqrt{\left(1-\eta^{2}\right)\left(k^{\prime \prime}+k^{2} \eta^{2}\right)}} \leq M
$$

We have

$$
Z^{+}=\frac{1}{\lambda^{\frac{3}{2}}} \int_{\eta(0)}^{1} F(\eta, \varepsilon, \varphi, \lambda) d \eta
$$

where

$$
F(\eta, \varepsilon, \varphi, \lambda)=\frac{\left(2 k \eta-\frac{\varepsilon p_{x}}{\sqrt{\lambda}}\right)^{2}\left(2 k^{2} \eta^{2}+p_{x}-\frac{\varepsilon^{2} p_{x}^{2}}{2 \lambda}\right)}{\sqrt{\left(1-\eta^{2}\right)\left(k^{\prime \prime}+k^{2} \eta^{2}\right)}}, \eta(0)=\frac{\varepsilon p_{x}}{2 k \sqrt{\lambda}} .
$$

We can write

$$
F(\eta, \varepsilon, \varphi, \lambda)=F_{1}(\eta, \varepsilon, \varphi, \lambda)+\frac{P\left(\eta, \frac{\varepsilon}{\sqrt{\lambda}}, \varphi\right)}{\sqrt{\left(1-\eta^{2}\right)\left(k^{\prime \prime}+k^{2} \eta^{2}\right)}}
$$

where

$$
F_{1}(\eta, \varepsilon, \varphi, \lambda)=\frac{4 k^{2} \eta^{2}\left(2 k^{2} \eta^{2}+p_{x}\right)}{\sqrt{\left(1-\eta^{2}\right)\left(k^{\prime \prime}+k^{2} \eta^{2}\right)}}
$$

and $P$ is polynomic in $\eta$ and $\frac{\varepsilon}{\sqrt{\lambda}}$ and can be written as

$$
P=\eta \frac{\varepsilon}{\sqrt{\lambda}} Q_{1}+\frac{\varepsilon^{2}}{\lambda} Q_{2} .
$$

Using $\left(H_{4}\right)$ and $(5.15)$ we get

$$
\int_{\eta(0)}^{1} F_{1}(\eta, \varepsilon, \varphi, \lambda) d \eta=\int_{0}^{1} F_{1}(\eta, 0, \varphi, \lambda) d \eta+\frac{|\varepsilon| K_{2}}{\sqrt{\lambda}}
$$

where $K_{2}$ is uniform. 
Moreover we have using $\left(H_{4}\right)$ :

$$
\frac{\varepsilon^{2}}{\lambda}\left|\int_{\eta(0)}^{1} \frac{Q_{2} d \eta}{\sqrt{\left(1-\eta^{2}\right)\left(k^{\prime \prime}+k^{2} \eta^{2}\right)}}\right| \leq \frac{|\varepsilon| K_{3}}{\sqrt{\lambda}}
$$

where $K_{3}$ is uniform.

Using $k^{\prime \prime}>0$ we can write

$$
\left|\int_{\eta(0)}^{1} \frac{\eta \frac{\varepsilon}{\sqrt{\lambda}} Q_{1} d \eta}{\sqrt{\left(1-\eta^{2}\right)\left(k^{\prime \prime}+k^{2} \eta^{2}\right)}}\right| \leq \frac{|\varepsilon|}{k \sqrt{\lambda}} \int_{\eta(0)}^{1} \frac{\left|Q_{1}\right| d \eta}{\sqrt{1-\eta^{2}}} .
$$

Hence we get

$$
\left|\int_{\eta(0)}^{1} \frac{\eta \frac{\varepsilon}{\sqrt{\lambda}} Q_{1} d \eta}{\sqrt{\left(1-\eta^{2}\right)\left(k^{\prime \prime}+k^{2} \eta^{2}\right)}}\right| \leq \frac{|\varepsilon| K_{4}}{\sqrt{\lambda}}
$$

where $K_{4}$ is uniform.

Therefore we prove

$$
Z^{+}=\frac{1}{\lambda^{\frac{3}{2}}}\left[I_{2}+\frac{|\varepsilon| K_{5}}{\sqrt{\lambda}}\right]
$$

where $K_{5}$ is uniform. The proposition is proved.

\subsection{Case C: estimation of the drift $Z^{\sigma}$}

In case $C$, we have

$$
k^{\prime \prime}=0, k=1, p_{x}=-1+\frac{\varepsilon^{2} p_{x}^{2}}{2 \lambda} .
$$

We assume $p_{x} \leq 0$. In this case we have two types of behaviors corresponding respectively to $\sigma=1$ and $\sigma=-1$. The projections of the geodesics in the $(x, y)$-plane are the elastica represented on figures 9,10 .

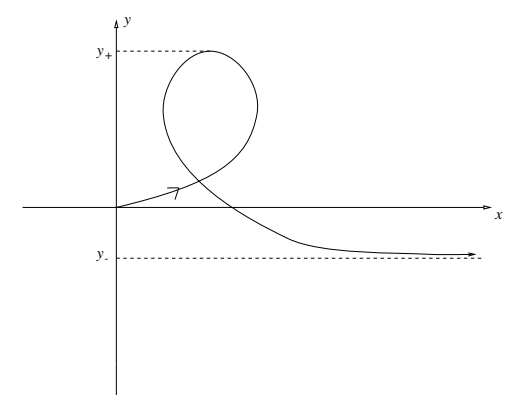

Figure 9. $\sigma=+1$

If $\sigma=+1$, then the geodesic intersects once the plane $y=0$ and if $\sigma=-1$, this intersection is empty. We can estimate the drift when $\sigma=+1$. We have

$$
Z^{+}=\frac{1}{\lambda^{\frac{3}{2}}} \int_{\eta(0)}^{1} \frac{\left(2 k \eta-\frac{\varepsilon p_{x}}{\sqrt{\lambda}}\right)^{2}\left(2 k^{2} \eta^{2}-1\right) d \eta}{k \eta \sqrt{1-\eta^{2}}}
$$

where $\eta(0)=\frac{\varepsilon p_{x}}{2 k \sqrt{\lambda}}$.

Hence we get: 


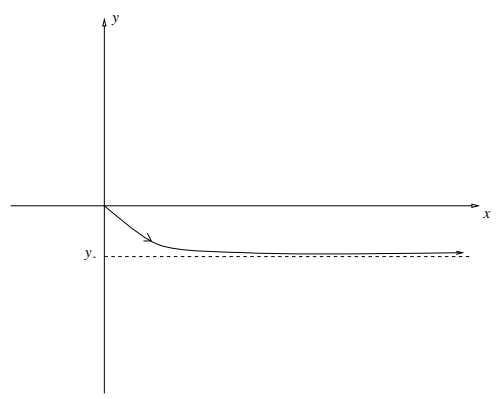

FiguRE 10. $\sigma=-1$

Proposition 5.14. Assume $k^{\prime \prime}=0, p_{x} \leq 0$, then we have

$$
Z^{+}=\frac{1}{\lambda^{\frac{2}{2}}}\left[I_{1}+O\left(\frac{1}{\sqrt{\lambda}}\right)\right]
$$

where $O\left(\frac{1}{\sqrt{\lambda}}\right) \rightarrow 0$ uniformly when $\lambda \rightarrow \infty$.

5.12. Case B: estimation of the Drift $Z^{\sigma}$

In case $B, \eta$ oscillates between $b$ and 1 or -1 and $-b$, where $b=\sqrt{-\frac{k^{\prime \prime}}{k^{2}}}$, according to the law

$$
\frac{\dot{\eta}^{2}}{\lambda}=\left(1-\eta^{2}\right)\left(k^{\prime \prime}+k^{2} \eta^{2}\right)
$$

where $k^{\prime \prime}<0$. Let us assume $p_{x} \leq 0$. If $\sigma=+1$, then $\eta$ moves first from $\eta(0)$ to 1 , if $\sigma=-1$, then $\eta$ moves first from $\eta(0)$ to $b$, where $\eta(0)=\frac{\varepsilon p_{x}}{2 k \sqrt{\lambda}}$. From figure 8 , we observe that when $\lambda \rightarrow \infty$ then $p_{x} \rightarrow-1$, in the domain $k^{\prime \prime}<0$. When $\sigma=+1$, the amplitude of the motion between the first two zeros of $y$ is $1-\eta(0)$ and tends to 1 when $\lambda \rightarrow \infty$. If $\sigma=-1$, this amplitude is $\eta(0)-b$ and tends to 0 when $\lambda \rightarrow \infty$. Therefore the estimations of $Z^{+}$ and $Z^{-}$are different. We represent on figures 11,12 the projection of the geodesics in the plane $(x, y)$.

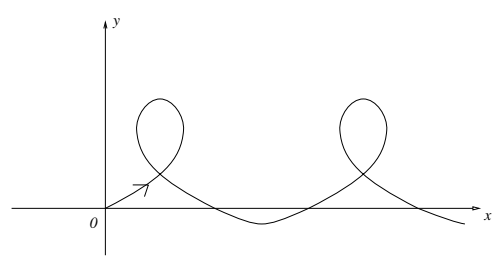

FiguRE 11. $\sigma=+1$ 


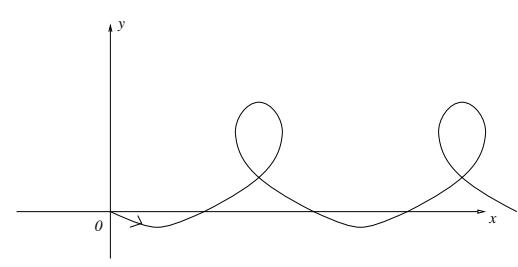

FiguRE 12. $\sigma=-1$

First we estimate the drift $Z^{\sigma}$ in the limit case $p_{x}=-1$. In this case the $y$ coordinate oscillates between $\left[y_{4}, y_{2}\right]$ where $y_{4}=y(0)=0$ and we have $\sigma=1$. The drift is given by

$$
Z^{+}=\int_{0}^{y^{+}} \frac{y^{2} P_{1}(y) d y}{\sqrt{1-P_{1}^{2}(y)}}
$$

where $\sqrt{1-P_{1}^{2}(y)}$ is zero at $y=0$. If we calculate in the $\eta$ coordinate, we get

$$
Z^{+}=\frac{1}{\lambda^{\frac{3}{2}}} \int_{\eta(0)}^{1} \frac{\left(2 k \eta+\frac{\varepsilon}{\sqrt{\lambda}}\right)^{2}\left(2 k^{2} \eta^{2}-1-\frac{\varepsilon^{2}}{2 \lambda}\right) d \eta}{k \sqrt{\left(1-\eta^{2}\right)(\eta-b)(\eta+b)}}
$$

where

$$
2 k^{2}=2+\frac{\varepsilon^{2}}{2 \lambda}, 2 k^{\prime \prime}=-\frac{\varepsilon^{2}}{2 \lambda}, b=\frac{\sqrt{-k^{\prime \prime}}}{k}=-\frac{\varepsilon}{2 k \sqrt{\lambda}} .
$$

Since

$$
\left(2 k \eta+\frac{\varepsilon}{\sqrt{\lambda}}\right)^{2}=4 k^{2}(\eta-b)^{2}
$$

we have

$$
Z^{+}=\frac{1}{\lambda^{\frac{3}{2}}} \int_{\eta(0)}^{1} \frac{4 k(\eta-b)^{\frac{3}{2}}\left(2 k^{2} \eta^{2}-1-\frac{\varepsilon^{2}}{2 \lambda}\right) d \eta}{\sqrt{\left(1-\eta^{2}\right)(\eta+b)}}
$$

and since $k \rightarrow 1$ when $\lambda \rightarrow \infty$, we get

$$
Z^{+}=\frac{1}{\lambda^{\frac{3}{2}}}\left[4 \int_{0}^{1} \frac{\eta\left(2 \eta^{2}-1\right) d \eta}{\sqrt{1-\eta^{2}}}+O\left(\frac{1}{\sqrt{\lambda}}\right)\right]
$$

where $O\left(\frac{1}{\sqrt{\lambda}}\right) \rightarrow 0$ uniformly when $\lambda \rightarrow \infty$. The same estimation is valid in general.

If $\sigma=-1$, we get

$$
Z^{-}=\frac{1}{\lambda^{\frac{3}{2}}} O\left(\frac{1}{\sqrt{\lambda}}\right)
$$

where $O\left(\frac{1}{\sqrt{\lambda}}\right) \rightarrow 0$ uniformly when $\lambda \rightarrow \infty$. Therefore we have: 
Proposition 5.15. Assume $k^{\prime \prime}<0, p_{x} \leq 0$. Then we have

$$
\begin{aligned}
& \text { (i) } Z^{+}=\frac{1}{\lambda^{\frac{3}{2}}}\left[I_{1}+O\left(\frac{1}{\sqrt{\lambda}}\right)\right] \\
& \text { (ii) } Z^{-}=\frac{1}{\lambda^{\frac{3}{2}}} O\left(\frac{1}{\sqrt{\lambda}}\right)
\end{aligned}
$$

where $O\left(\frac{1}{\sqrt{\lambda}}\right) \rightarrow 0$ uniformly when $\lambda \rightarrow \infty$.

REMARK 5.16. We have identified a basic invariant of the problem which is $I_{1}=4 / 3$ and which is given by the flat case as $I_{1}=\lim _{k^{\prime} \rightarrow 0} I_{2}$.

Theorem 5.17. Let $M \geq 0$. Then the abnormal geodesic $\gamma: t \mapsto( \pm t, 0,0)$ is $C^{0}$-isolated in the set of geodesics of length less than $M$.

Proof. Let $(\varphi, \lambda) \in S^{1} \times \mathbb{R}^{+}$be a parameter and $e:[0, T] \mapsto U$ be the corresponding geodesic of length $T$. Assume $e \neq \gamma$ and $e(T) \in \operatorname{Im} \gamma$. Let $0<t_{1}<\ldots<t_{N} \leq M$ be the times such that $y\left(t_{i}\right)=0$. Assume first $t_{1}=T$ and let $Z^{\sigma}$ be the corresponding drift. From our analysis we have the following.

If $k^{\prime \prime}<0, \sigma=-1, Z^{\sigma}<0$, otherwise we have

$$
Z^{\sigma}=\frac{1}{\lambda^{\frac{3}{2}}}\left[d+O\left(\frac{1}{\sqrt{\lambda}}\right)\right]
$$

where $d \geq m>0$ and $O\left(\frac{1}{\sqrt{\lambda}}\right) \rightarrow 0$ uniformly when $\lambda \rightarrow \infty$.

Let $Y=\sup _{t \in[0, T]}|y(t)|$. Using lemmas 5.9 and 5.11, if $Y$ is small enough we must have

$$
p_{x}<0 \text { and }\left|p_{x}\right| \leq p_{z} Y^{2} .
$$

Using the previous estimates we have

$$
\left|\frac{\varepsilon p_{x}}{\sqrt{\lambda}}\right| \leq K_{1} Y
$$

where $K_{1}$ is uniform. If $k^{\prime \prime} \neq 0$ and $\sigma \neq 1$, we have

$$
y_{ \pm}=\frac{1}{\sqrt{\lambda}}\left( \pm 2 k-\frac{\varepsilon p_{x}}{\sqrt{\lambda}}\right)
$$

where $k \geq 1 / \sqrt{2}$. Hence if $\left|y_{ \pm}\right| \leq Y$ we must have for $Y$ small enough

$$
\frac{1}{\sqrt{\lambda}} \leq K_{2} Y
$$

where $K_{2}$ is uniform. Hence $\lambda \rightarrow \infty$ uniformly when $Y \rightarrow 0$. This proves that we cannot have $e\left(t_{1}\right) \in \operatorname{Im} \gamma$ if $Y$ is small enough.

For $p>1$, the drift $z\left(t_{p}\right)$ is given by the following relations.

If $k^{\prime \prime}<0, p=2 q$ or $2 q+1$ :

$$
z\left(t_{p}\right)=\frac{q}{\lambda^{\frac{3}{2}}}\left[I_{1}+O\left(\frac{1}{\sqrt{\lambda}}\right)\right]
$$

where $I_{1}=4 / 3$.

If $k^{\prime \prime}>0$

$$
z\left(t_{p}\right)=\frac{p}{\lambda^{\frac{3}{2}}}\left[I_{2}\left(p_{x}\right)+O\left(\frac{1}{\sqrt{\lambda}}\right)\right]
$$

where inf $I_{2}>0$ for $p_{x} \leq 0$. Moreover $\lambda \rightarrow \infty$ uniformly when $Y \rightarrow 0$.

Hence in general we cannot have $e(T) \in \operatorname{Im} \gamma$ if $Y$ is small enough. 


\section{Conclusion}

The analysis of the flat case is almost complete. The only missing point is the description of the singularities of the conjugate locus when $k \rightarrow 0$ and $k \rightarrow 1$. For this we need to compute the first conjugate time $t_{1 c}$ along a geodesic. The numerical simulations show that $\frac{t_{1 c} \sqrt{\lambda}}{3 K} \sim 0.97$ but it is not sufficient. Nevertheless no doubt that the conjugate locus has singularities similar to the sphere and is in particular not sub-analytic when $k \rightarrow 1$.

In the last section we have outlined the analysis of a one parameter perturbation of the flat case which is relevant to analyze the generic integrable case where $g=a(y) d x^{2}+c(y) d y^{2}$ which is studied in [7], where the spheres are described. This analysis is not trivial. In particular we must generalize the parametrizations of the geodesics using elliptic integrals etablished in the flat case.

This work explains how accurate is the flat case to describe the generic case. In particular we can use the analysis of section 5 to indicate what are the stable and the unstable properties.

The stable properties are the following. In the flat case, if we relate the geodesics to the pendulum (or the elastica), the role of the abnormal geodesics is to create geodesics which are close to the separatrix. This phenomenon is persistent in the perturbed case. Moreover the separatrix codes admissible geodesics corresponding to $k^{\prime \prime}=0$. This phenomenon is the origin of the non sub-analyticity of the sphere. This can be analyzed in the general case, using asymptotic expansions where the invariant $I_{1}=4 / 3$ plays an important role, see [7].

A basic unstable property is the following. Observe that in section 5 , if $\varepsilon \neq$ 0 there exists geodesics related to a rotating pendulum (or a non inflexional elastica) which correspond to the case $B$, where $k^{\prime \prime}<0$. They generate the following phenomenon. When $\varepsilon=0$, the geodesic corresponding to a first ascending branch $y$ associated to $\varphi \in\left[-\frac{\pi}{2}, \frac{\pi}{2}\right]$ intersects at $y=0$ the one corresponding to a descending branch given by $\pi-\varphi$. When $\varepsilon \neq 0$ this is no longer true. The time between the first two intersections with $y=0$ is

$$
\theta^{\sigma}=\frac{2}{\sqrt{\lambda}} \int_{\eta(0)}^{\eta_{\sigma}} \frac{\sigma d \eta}{\sqrt{\left(1-\eta^{2}\right)\left(k^{\prime \prime}+k^{2} \eta^{2}\right)}}
$$

and the case $\sigma=+1$ differs from $\sigma=-1$. This is illustrated by the figures 13 and 14 below on which we represent on the set of parameters $\left(p_{x}, \lambda\right)$ the values corresponding to $\theta^{\sigma} \leq r$, where $r$ is small enough (we have supposed $\varepsilon<0)$.

In the flat case we are always in the situation described by $\sigma=-1$ and in the domain $k^{\prime \prime}>0$. In particular the case $\sigma=+1$ is at the origin of a portion of the wave front not present in the flat case. Other unstability phenomenon are consequences of the existence of the geodesics corresponding to $k^{\prime \prime}<0$. See [7] for a more complete discussion. Endly the analysis of the generic case where the set of geodesics is not integrable is outlined in [9]. 


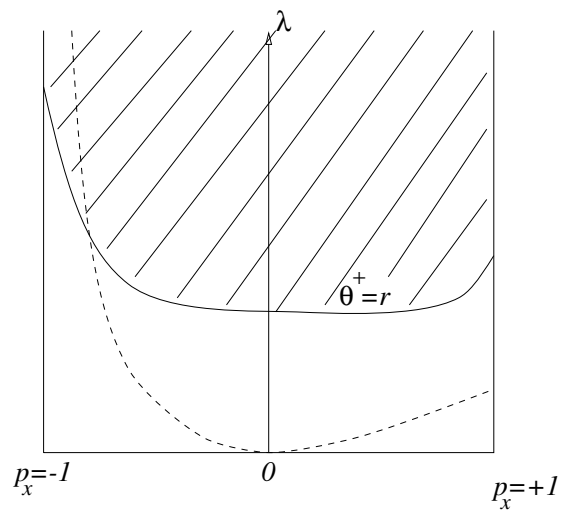

FiguRE 13. $\sigma=+1$

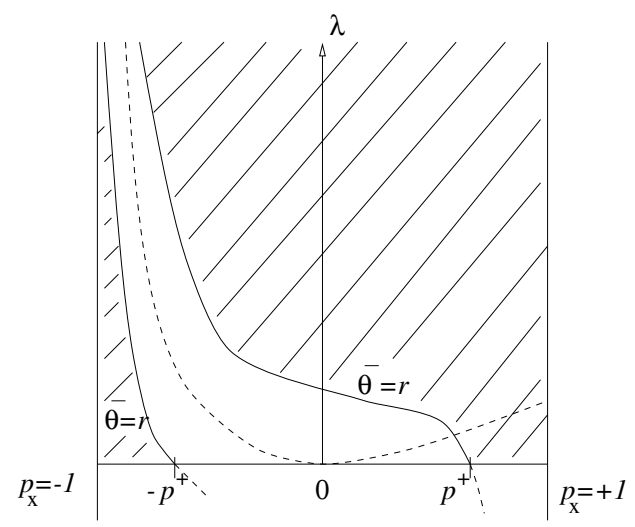

FIGURE 14. $\sigma=-1$

\section{REFERENCES}

[1] A. Agrachev, A. V. Sarychev: Strong minimality of abnormal geodesics for 2distributions, Journal of Dynamical and control systems, 2, 1995, 139-176.

[2] A. Agrachev: Exponential mappings for contact sub-Riemannian structures, Journal of dynamical and Control Systems, 2, 1996, 321-358.

[3] A. Agrachev: Any smooth simple $\mathrm{H}^{1}$-local length minimizer in the Carnot-Caratheodory space is a $\mathrm{C}^{0}$-local minimizer, Preprint of Laboratoire de Topologie, Dijon, 1996.

[4] V.I. Arnold: Méthodes mathématiques pour la mécanique classique, Éditions MIR, Moscou, 1976.

[5] G.A. Bliss: Lectures on the calculus of variations, The University of Chicago Press, 1946.

[6] B. Bonnard: Feedback equivalence for nonlinear systems and the time optimal control problem, SIAM J. on Control and Opt., 29, 1991, 1300-1321.

[7] B. Bonnard, M. Chyba: Exponential mapping, sphere and waves front in SRgeometry: the generic integrable Martinet case, Preprint of Laboratoire de Topologie, Dijon, 1997.

[8] B. Bonnard, M. Chyba, H. Heutte: Contrôle optimal géométrique appliqué, Preprint of Laboratoire de Topologie, Dijon, 1995.

[9] B. Bonnard, M. Chyba, I. Kupka: Non-integrable geodesics in SR Martinet geometry, in Proceedings AMS conference, Boulder, 1997. 
[10] B. Bonnard, M. Chyba, E. Trélat: Sub-Riemannian geometry: one parameter deformation of the Martinet flat case, to appear in Journal of Dynamical and Control Systems.

[11] R. W. Brockett: Control theory and singular Riemannian geometry, in New directions in applied Math., Springer-Verlag, New-York, 1981.

[12] E. Cartan: Leçons sur la géomètrie des espaces de Riemann, Ed. J. Gabay, Paris, 1988.

[13] H. Davis: Introduction to non linear differential and integral equation, Dover, NewYork, 1962.

[14] J. Dieudonné: Calcul Infinitésimal, Hermann, Paris, 1980.

[15] M. Do Carmo: Riemannian geometry, Birkhauser, Boston, 1992.

[16] L. V .D. Dries, A. Macintyre, D. Marker: The elementary theory of restricted analytic fields with exponentiation, Annals of Mathematics, 140, 1994, 183-205.

[17] C. El Alaowi, J. P. Gauthier, I. Kupka: Small sub-Riemannian balls on $R^{3}$, Journal of dynamical and Control Systems, 2, 1996, 359-421.

[18] R. Gérard, H. Tahora: Singular nonlinear PDE, Vieweg-Verlag, Germany, 1996.

[19] J. Gregory: Quadratic form theory and differential equation, Academic Press, NewYork, 1980.

[20] U. Hamenstadt: Some regularity theorem for Carnot-Caratheodory metrics, J. Differential geometry, 32, 1991, 819-850.

[21] F. John: Partial differential equations, Springer-Verlag, New-York, 1971.

[22] A. G. Khovanskii: Fewnomials, Trans. AMS, 88, 1991.

[23] I. Kupka: Abnormal extremals, Preprint, 1992.

[24] I. Kupka: Géométrie sous-Riemannienne, in Séminaire Bourbaki, 1996.

[25] D .F. Lawden: Elliptic functions and applications, Springer-Verlag, New-York, 1989.

[26] E. B. Lee, L. Markus: Foundations of optimal control theory, John Wiley and Sons, New-York, 1967.

[27] J. M. Lion, J. P. Rolin: Théorèmes de préparation pour les fonctions logarithmoexponentielles, Annales de l'Institut Fourier, 47, 1997, 859-884.

[28] W. S. Liu and H. J. Susmann: Shortest paths for sub-Riemannian metrics of rank two distributions, to appear in Trans. AMS.

[29] S. Lojasiewicz, H. J. Sussmann: Some examples of reachable sets and optimal cost functions that fail to be subanalytic, SIAMJ. Control and Optimization, 23, 1985, 584-598.

[30] A. E. H. Love: A treatise of the mathematical theory of elasticity, Dover, 1944.

[31] S. B. Myers: Connections between differential geometry and topology, Duke Math. J., 1, 1935, 376-391.

[32] L. Pontriaguine et al:: Théorie mathématique des processus optimaux, Éditions MIR, Moscou, 1974.

[33] W. Respondek, M. Zhitomirskii: Feedback classification of nonlinear control systems on 3-manifolds, to appear in Math. Control Systems and Signals.

[34] M. Spivak: Differential geometry, Publish on Perish, Inc., Berkeley, 1979.

[35] R. Strichartz: Sub-Riemannian geometry, J. Differential geometry, 24, 1986, 221-263.

[36] J. Tannery, J. Molk: Eléments de la théorie des fonctions elliptiques, Tomes I à IV, Gauthier-Villars, Paris, 1896.

[37] A. Weinstein: The cut-locus and conjugate-locus of a Riemannian manifold, Annals of Maths, 87, 1968, 29-41.

[38] E. T. Whittaker, G. N. Watson: A course of modern analysis, Cambridge U. Press, New York, 1927. 


\section{Appendix A. Martinet Flat Case}

\section{A.1. Geodesics}

On the three first pictures we have drawn the projection in the plane $(x, y)$ of the different types of normal bi-extremals which appear in Martinet flat case for $\lambda \neq 0$. The figures $15,16,17$ represent respectively the curves $t \mapsto(x(t), y(t))$ corresponding to initial value $(\varphi, \lambda)$ equals to $(0.3,50),(-$ $0.4,35)$ and $(-0.9,80)$. Recall the parametrization of these geodesics,

$$
\begin{aligned}
& x(t)=-t+\frac{2}{\sqrt{\lambda}}(\mathrm{E}(u)-\mathrm{E}(\mathrm{K})) \\
& y(t)=-\frac{2 k}{\sqrt{\lambda}} \mathrm{cn} u \\
& z(t)=\frac{2}{3 \lambda^{\frac{3}{2}}}\left[\left(2 k^{2}-1\right)(\mathrm{E}(u)-\mathrm{E}(\mathrm{K}))+k^{\prime 2} t \sqrt{\lambda}+2 k^{2} \operatorname{sn} u \operatorname{cn} u \operatorname{dn} u\right]
\end{aligned}
$$

where $u=K+t \sqrt{\lambda}, \varphi \in]-\frac{\pi}{2}, \frac{\pi}{2}[, \lambda>0$. From the second equation, we can deduce that the $y$-coordinate oscillates between $y_{+},-y_{+}$where $y_{+}=$ $\max y(t)$ is given by $y_{+}=2 k / \sqrt{\lambda}$.

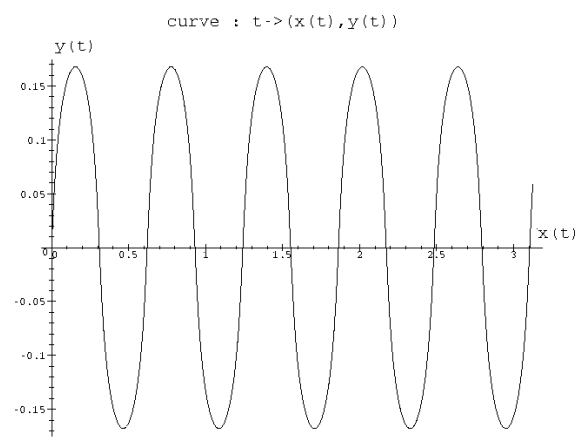

FiguRE 15

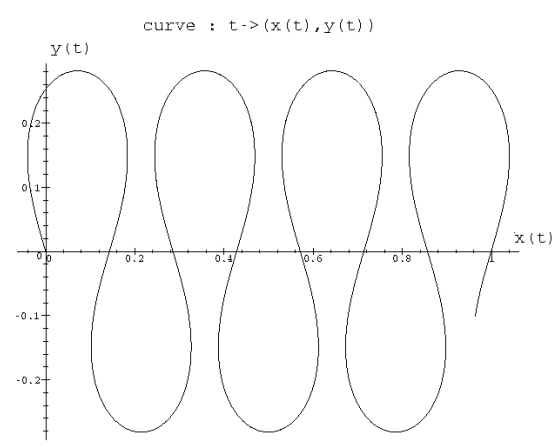

Figure 16

On figures $18,19,20$ we represent the curves $t \mapsto(x(t), y(t), z(t))$. Observe that $t \mapsto y(t)$ is periodic of period $4 K$ and $t \mapsto(x(t), z(t))$ are a sum of a periodic function and a linear one: there is a shift after one period. 


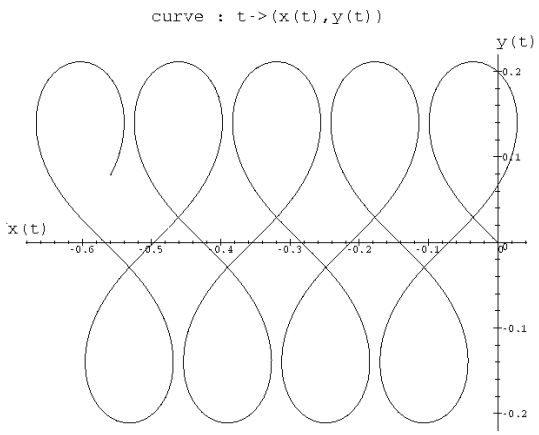

FiguRE 17

The average behavior is obtained by taking the respective lines joining 0 to $(2 K, x(2 K)),(2 K, z(2 K))$. Initial value is taken as $(\varphi, \lambda)=(0.3,50)$.

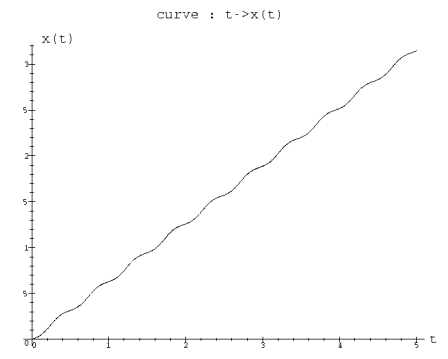

FiguRE 18

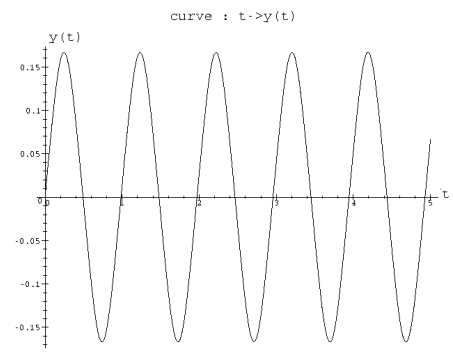

FiguRE 19

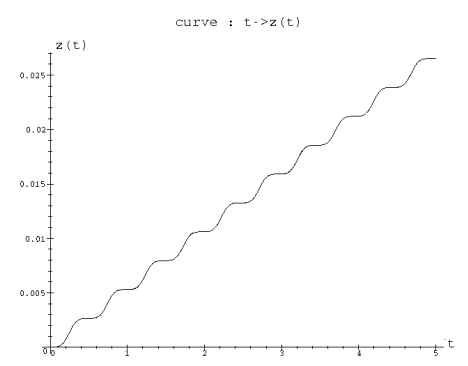

FiguRE 20 


\section{A.2. Conjugate points}

On figures $21,22,23$ we have stopped the numerical integration at the first conjugate point $\left(x\left(t_{1 c}\right), y\left(t_{1 c}\right)\right)$ computed using the variational equation. These pictures correspond to the initial values $(\varphi, \lambda)$ chosen for figures 15 , 16,17 . The conjugate times $t_{1 c}$ are respectively $0.72,1.02$ and 0.53 .

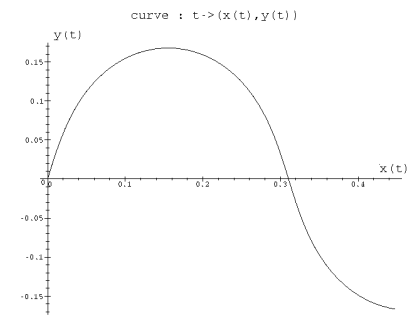

FiguRE 21

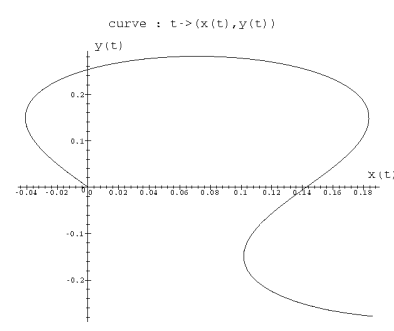

FIGURE 22

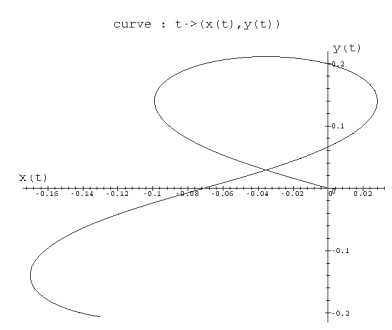

FIGURE 23

We observe experimentally on figure 24 the following phenomenon. If we set $v_{1 c}=t_{1 c} \sqrt{\lambda}$, the quotient $\frac{v_{1 c}}{3 K}$ where $4 K$ is the period of $t \rightarrow y(t)$ is roughly a constant $\simeq 0.97$.

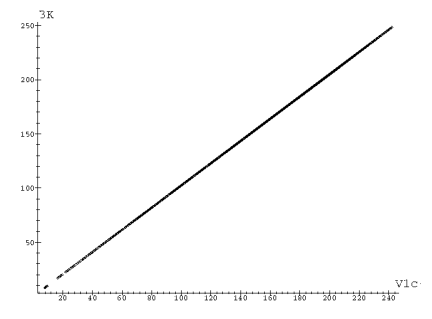

FiguRE 24 


\section{A.3. Cut locus}

On figure 25 we have represented, for $z>0$, the intersection of the cut locus with the sphere with radius $r=0.1$. The complete curve is the union of this curve and of the symmetric one with respect to the origine. It was established that the cut locus is contained in Martinet surface. Hence this intersection belongs to the plane $(x, z)$, the horizontal axis being the $x$-axis. The maximum of this curve is reached at $\varphi=0$.

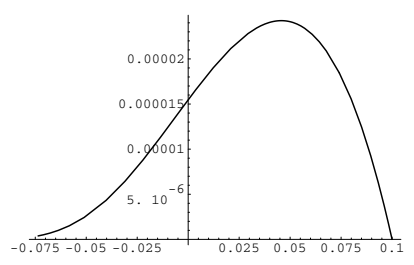

FIGURE 25

\section{A.4. Sphere}

The figure 26 shows the sphere of radius $r=0.1$ and the figure 27 represents a projection of this sphere on the plane $O x z$. The central black curve is the set of points corresponding to $\lambda=0$. The points above this curve correspond to $\lambda>0$ and below to $\lambda<0$. The projection on the plane $O x y$ is a disk with radius $r=0.1$ (it is not represented).

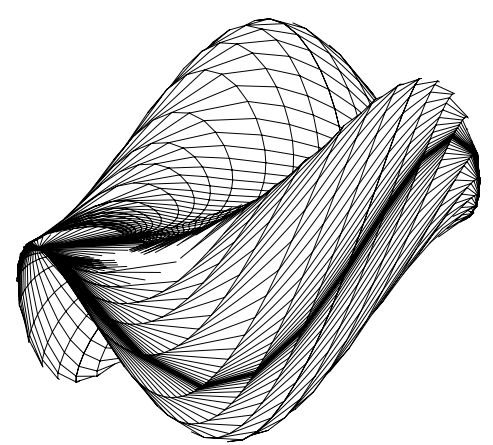

FiguRE 26

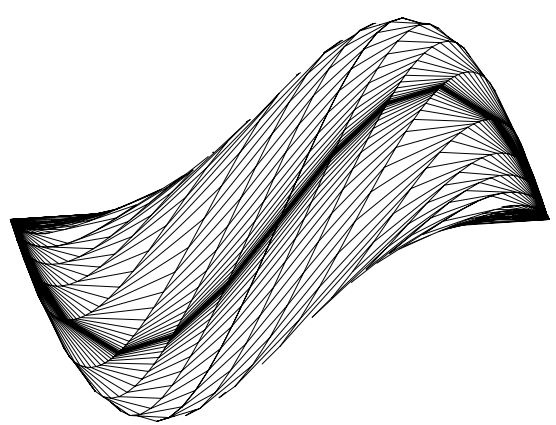

FiguRE 27 
The figure 28 represents the conjugate locus for values of $|\lambda|$ contained in $\left[10^{5}, 4 * 10^{6}\right]$, the black line represents the abnormal extremal. The figure 29 describes the following phenomenon. Assume $\lambda>0$. The shape of the conjugate locus is different if $\varphi \rightarrow \frac{\pi}{2}$ or $\varphi \rightarrow-\frac{\pi}{2}$. Indeed the first conjugate time satisfies $2 K<t_{1 c} \sqrt{\lambda}<3 K$ and when $\varphi$ decreases from $\frac{\pi}{2}$ to $-\frac{\pi}{2}$, the graph of $\mathrm{K}$ increases from $\frac{\pi}{2}$ to $+\infty$. Hence when $\varphi \rightarrow \frac{\pi}{2}, t_{1 c} \sqrt{\lambda}$ is bounded by $\frac{3 \pi}{2}$ and when $\varphi \rightarrow-\frac{\pi}{2}, t_{1 c} \sqrt{\lambda} \rightarrow+\infty$. (For numerical reasons the conjugate locus is not represented when $\varphi \rightarrow \frac{\pi}{2}$ and $\varphi \rightarrow-\frac{\pi}{2}$ ).

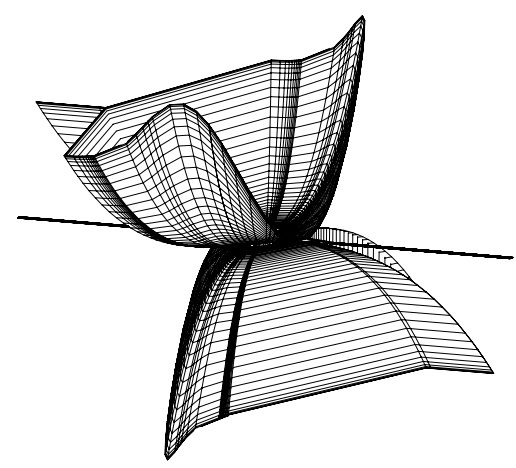

FIGURE 28

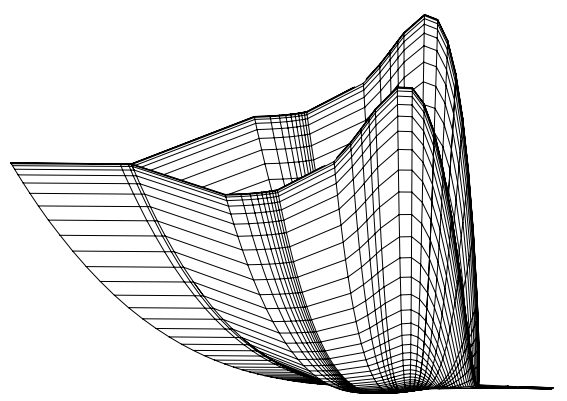

FiguRE 29

\section{Appendix B. One parameter deformation of the Martinet FLAT CASE \\ B.1. Geodesics}

On figures 30 and 31 we show the projection on the plane $(x, y)$ of geodesics corresponding to the cases $\mathrm{A}\left(k^{\prime \prime}>0\right)$ and $\mathrm{B}\left(k^{\prime \prime}<0\right)$. 


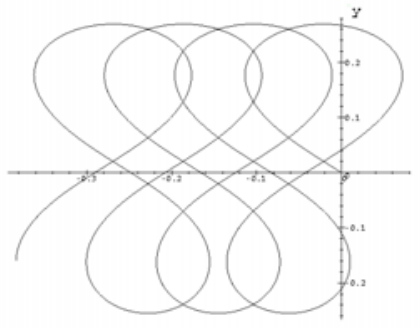

Figure 30

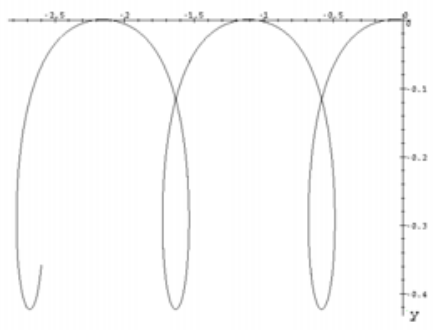

FiguRe 31

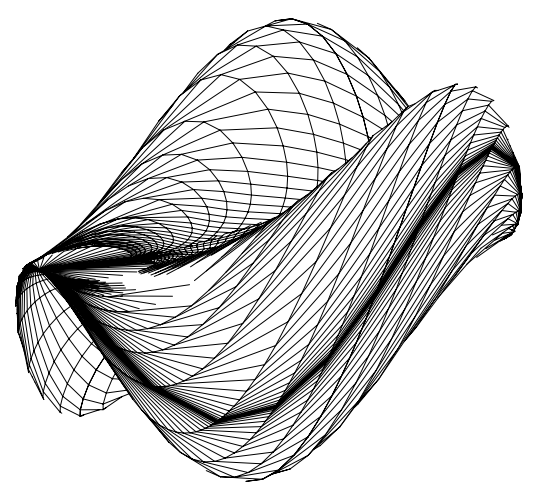

Figure 32

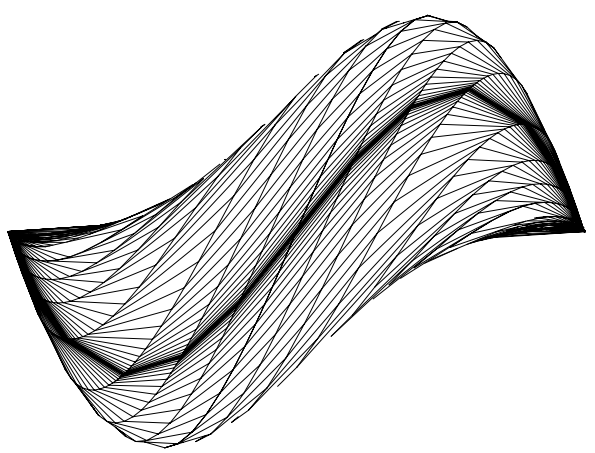

FIGURE 33

\section{B.2. Sphere}

The last two pictures represent the sphere of radius $r=0.1$ computed for $\varepsilon=0.5$.

Esaim: CoCv, December 1997, Vol. 2, Pp. $377-448$ 